\title{
Equilibrium Structure in the Presence of Methyl Internal Rotation: Microwave Spectroscopy and Quantum Chemistry Study of the Two Conformers of 2-Acetylfuran*
}

\author{
Christina Dindića ${ }^{a}$ Arne Lüchowa ${ }^{\mathrm{a}}$, Natalja Vogt ${ }^{\mathrm{b}, \mathrm{c}}$, Jean Demaison ${ }^{\mathrm{b}}$, Ha Vinh Lam Nguyen ${ }^{\mathrm{d}, \mathrm{e}^{* *}}$ \\ a Institute of Physical Chemistry, RWTH Aachen University, Landoltweg 2, 52074 Aachen, \\ Germany \\ ${ }^{b}$ Section of Chemical Information Systems, University of Ulm, Albert-Einstein-Allee 47, 89069 Ulm, \\ Germany \\ c Department of Chemistry, Lomonosov Moscow State University, 119991 Moscow, Russian Federation \\ a Laboratoire Interuniversitaire des Systèmes Atmosphériques (LISA), CNRS UMR 7583, Université Paris- \\ Est Créteil, Université de Paris, Institut Pierre Simon Laplace, 61 avenue du Général de Gaulle, 94010 \\ Créteil, France \\ e Institut Universitaire de France (IUF), 1 rue Descartes, 75231 Paris cedex 05, France \\ * In memory of Wolfgang Stahl \\ ** Corresponding author. Email: lam.nguyen@lisa.ipsl.fr
}


Table S1a: Nuclear coordinates in the principal axis system of anti-2AF and syn-2AF calculated at the MP2/6-311++G(d,p) level of theory (for atom numbering see Figure 1). The structure optimizations were carried out first with full-symmetry relaxation and finally for $\mathrm{C}_{s}$ symmetry (opt=verytight).

\begin{tabular}{|c|c|c|c|c|c|c|}
\hline & \multicolumn{3}{|c|}{ anti-2AF } & \multicolumn{3}{|c|}{ syn-2AF } \\
\hline & $a / \AA$ & $b / \AA$ & $c / \AA$ & $a / \AA$ & $b / \AA$ & $c / \AA$ \\
\hline 01 & 0.715984 & -1.045023 & 0.000000 & 0.814705 & -1.042989 & 0.000000 \\
\hline $\mathrm{C} 2$ & 0.051541 & 0.148445 & 0.000000 & 0.045911 & 0.078528 & 0.000000 \\
\hline C3 & 0.955769 & 1.191884 & 0.000000 & 0.854426 & 1.202104 & 0.000000 \\
\hline C4 & 2.247673 & 0.597436 & 0.000000 & 2.196007 & 0.729066 & 0.000000 \\
\hline C5 & 2.042607 & -0.762492 & 0.000000 & 2.107239 & -0.643123 & 0.000000 \\
\hline C6 & -1.422266 & 0.125699 & 0.000000 & -1.417796 & -0.097733 & 0.000000 \\
\hline 07 & -2.046420 & 1.179473 & 0.000000 & -1.928194 & -1.209288 & 0.000000 \\
\hline C8 & -2.090746 & -1.230285 & 0.000000 & -2.232606 & 1.180070 & 0.000000 \\
\hline H9 & 2.705835 & -1.614404 & 0.000000 & 2.842788 & -1.433495 & 0.000000 \\
\hline H10 & 3.207501 & 1.093792 & 0.000000 & 3.106969 & 1.310031 & 0.000000 \\
\hline H11 & 0.693267 & 2.239893 & 0.000000 & 0.517211 & 2.229037 & 0.000000 \\
\hline H12 & -1.783984 & -1.801361 & 0.881399 & -1.998230 & 1.779074 & -0.885289 \\
\hline H13 & -3.172299 & -1.092036 & 0.000000 & -3.292112 & 0.922763 & 0.000000 \\
\hline H14 & -1.783984 & -1.801361 & -0.881399 & -1.998230 & 1.779074 & 0.885289 \\
\hline
\end{tabular}

Table S1b: Nuclear coordinates in the principal axis system of anti-2AF and syn-2AF calculated at the B2PLYP-D3/6-311+G(3df,2pd) level of theory (for atom numbering see Figure 1). The optimizations were done under symmetry constraint.

\begin{tabular}{|c|c|c|c|c|c|c|}
\hline & \multicolumn{3}{|c|}{ anti-2AF } & \multicolumn{3}{|c|}{ syn-2AF } \\
\hline & $a / \AA$ & b / A & $c / \AA$ & $a / \AA$ & $\boldsymbol{b} / \boldsymbol{A}$ & $c / \AA$ \\
\hline 01 & 0.722641 & -1.043120 & 0.000000 & 0.822843 & 1.039229 & 0.000000 \\
\hline $\mathrm{C} 2$ & 0.048657 & 0.150448 & 0.000000 & 0.043717 & -0.079873 & 0.000000 \\
\hline C3 & 0.944378 & 1.183310 & 0.000000 & 0.843473 & -1.193026 & 0.000000 \\
\hline C4 & 2.238041 & 0.597029 & 0.000000 & 2.186674 & -0.729790 & 0.000000 \\
\hline C5 & 2.042573 & -0.751969 & 0.000000 & 2.108212 & 0.630896 & 0.000000 \\
\hline C6 & -1.416715 & 0.125260 & 0.000000 & -1.411957 & 0.099979 & 0.000000 \\
\hline 07 & -2.042755 & 1.170540 & 0.000000 & -1.919359 & 1.205067 & 0.000000 \\
\hline $\mathrm{C} 8$ & -2.085606 & -1.226131 & 0.000000 & -2.235744 & -1.168186 & 0.000000 \\
\hline H9 & 2.711149 & -1.592646 & 0.000000 & 2.848706 & 1.409078 & 0.000000 \\
\hline H10 & 3.187319 & 1.101165 & 0.000000 & 3.085974 & -1.318245 & 0.000000 \\
\hline H11 & 0.685010 & 2.226156 & 0.000000 & 0.507419 & -2.214246 & 0.000000 \\
\hline H12 & -1.780872 & -1.798420 & 0.875434 & -3.287310 & -0.902073 & -0.879737 \\
\hline H13 & -3.161448 & -1.088176 & 0.000000 & -2.010912 & -1.770228 & 0.000000 \\
\hline H14 & -1.780872 & -1.798420 & -0.875434 & -2.010912 & -1.770228 & 0.879737 \\
\hline
\end{tabular}


Table S2: Equilibrium rotational constants, $X_{\mathrm{e}}(X=A, B, C$ of anti-2AF calculated at various levels of theory. The deviations $\Delta X$ between the calculated and the ground state experimental values $X_{0}$ are given in $\mathrm{MHz}$. The relative deviations of the semi-experimental equilibrium structures with respect to $X_{\mathrm{e}}^{\mathrm{BO}}$ are given in \%. All optimizations were done under full-symmetry relaxation, except for the coupled cluster calculations assuming $\mathrm{C}_{\mathrm{s}}$ symmetry.

\begin{tabular}{|c|c|c|c|c|c|c|}
\hline Method/Basis Set & $\begin{array}{c}\text { A / } \\
\mathrm{MHz}\end{array}$ & $\begin{array}{l}\Delta \boldsymbol{A} / \\
\mathrm{MHz} \\
\text { or } \%\end{array}$ & $\begin{array}{c}B / \\
\mathrm{MHz}\end{array}$ & $\begin{array}{c}\Delta B / \\
M H z \\
\text { or \% }\end{array}$ & $\begin{array}{c}C / \\
\mathrm{MHz}\end{array}$ & $\begin{array}{c}\Delta C / \\
\mathrm{MHz} \\
\text { or } \%\end{array}$ \\
\hline MP2/6-31G(d,p) & 4820.6 & -31.5 & 1632.4 & 1.2 & 1228.7 & -1.9 \\
\hline$M P 2 / 6-31+G(d, p)$ & 4814.4 & -37.7 & 1626.8 & -4.4 & 1225.1 & -5.5 \\
\hline MP2/6-31++G(d,p) & 4814.1 & -38.0 & 1626.9 & -4.3 & 1225.1 & -5.4 \\
\hline MP2/6-311G(d,p) & 4824.1 & -28.0 & 1633.0 & 1.8 & 1229.3 & -1.3 \\
\hline MP2/6-311+G(d,p) & 4820.6 & -31.6 & 1630.5 & -0.7 & 1227.7 & -2.9 \\
\hline $\mathrm{MP} 2 / 6-311++\mathrm{G}(\mathrm{d}, \mathrm{p})$ & 4820.6 & -31.6 & 1630.5 & -0.7 & 1227.7 & -2.9 \\
\hline MP2/6-311G(df,pd) & 4855.6 & 3.4 & 1643.5 & 12.2 & 1237.2 & 6.6 \\
\hline MP2/6-311+G(df,pd) & 4854.8 & 2.7 & 1640.0 & 8.8 & 1235.2 & 4.6 \\
\hline MP2/6-311++G(df,pd) & 4854.3 & 2.2 & 1640.1 & 8.8 & 1235.2 & 4.6 \\
\hline MP2/6-311G(2d,2p) & 4839.6 & -12.5 & 1638.5 & 7.3 & 1233.3 & 2.7 \\
\hline MP2/6-311+G(2d,2p) & 4838.8 & -13.3 & 1636.3 & 5.1 & 1232.0 & 1.4 \\
\hline MP2/6-311++G(2d,2p) & 4838.7 & -13.4 & 1636.3 & 5.1 & 1232.0 & 1.4 \\
\hline MP2/6-311G(2df,2pd) & 4860.8 & 8.7 & 1648.5 & 17.3 & 1240.3 & 9.7 \\
\hline MP2/6-311+G(2df,2pd) & 4860.1 & 8.0 & 1645.5 & 14.3 & 1238.6 & 8.0 \\
\hline $\mathrm{MP} 2 / 6-311++\mathrm{G}(2 \mathrm{df}, 2 \mathrm{pd})$ & 4859.8 & 7.7 & 1645.6 & 14.4 & 1238.7 & 8.1 \\
\hline MP2/6-311G(3df,3pd) & 4862.8 & 10.7 & 1646.3 & 15.0 & 1239.2 & 8.6 \\
\hline MP2/6-311+G(3df,3pd) & 4859.7 & 7.6 & 1643.9 & 12.6 & 1237.7 & 7.1 \\
\hline MP2/6-311++G(3df,3pd) & 4859.7 & 7.6 & 1643.9 & 12.6 & 1237.7 & 7.1 \\
\hline MP2/cc-pVDZ & 4777.9 & -74.3 & 1622.9 & -8.3 & 1220.7 & -9.9 \\
\hline MP2/cc-pVTZ & 4850.8 & -1.4 & 1643.3 & 12.1 & 1236.8 & 6.2 \\
\hline MP2/aug-cc-pVDZ & 4766.4 & -85.7 & 1615.3 & -16.0 & 1215.6 & -15.0 \\
\hline MP2/aug-cc-pVTZ & 4849.1 & -3.0 & 1642.1 & 10.8 & 1236.0 & 5.4 \\
\hline B3LYP-D3/6-31G(d,p) & 4831.6 & -20.5 & 1629.1 & -2.1 & 1227.5 & -3.1 \\
\hline B3LYP-D3/6-31+G(d,p) & 4831.2 & -20.9 & 1624.8 & -6.4 & 1225.1 & -5.5 \\
\hline B3LYP-D3/6-31++G(d,p) & 4831.3 & -20.8 & 1624.7 & -6.5 & 1225.0 & -5.6 \\
\hline B3LYP-D3/6-311G(d,p) & 4851.3 & -0.8 & 1632.3 & 1.1 & 1230.6 & 0.0 \\
\hline B3LYP-D3/6-311+G(d,p) & 4853.0 & 0.9 & 1630.6 & -0.6 & 1229.8 & -0.8 \\
\hline B3LYP-D3/6-311++G(d,p) & 4852.8 & 0.7 & 1630.6 & -0.6 & 1229.8 & -0.8 \\
\hline B3LYP-D3/6-311G(df,pd) & 4866.5 & 14.4 & 1636.7 & 5.5 & 1234.1 & 3.5 \\
\hline B3LYP-D3/6-311+G(df,pd) & 4867.9 & 15.8 & 1634.6 & 3.4 & 1233.0 & 2.4 \\
\hline B3LYP-D3/6-311++G(df,pd) & 4867.7 & 15.6 & 1634.7 & 3.5 & 1233.0 & 2.4 \\
\hline B3LYP-D3/6-311G(3df,3pd) & 4878.7 & 26.6 & 1638.7 & 7.5 & 1236.0 & 5.4 \\
\hline B3LYP-D3/6-311+G(3df,3pd) & 4877.4 & 25.3 & 1637.0 & 5.8 & 1234.9 & 4.3 \\
\hline
\end{tabular}




\begin{tabular}{|c|c|c|c|c|c|c|}
\hline B3LYP-D3/6-311++G(3df,3pd) & 4877.4 & 25.3 & 1637.0 & 5.8 & 1234.9 & 4.3 \\
\hline B3LYP-D3/cc-pVDZ & 4821.0 & -31.1 & 1627.2 & -4.0 & 1225.9 & -4.7 \\
\hline B3LYP-D3/cc-pVTZ & 4872.8 & 20.7 & 1636.7 & 5.5 & 1234.4 & 3.8 \\
\hline B3LYP-D3/aug-cc-pVDZ & 4827.5 & -24.6 & 1623.5 & -7.7 & 1224.2 & -6.4 \\
\hline B3LYP-D3/aug-cc-pVTZ & 4873.2 & 21.1 & 1636.1 & 4.9 & 1234.1 & 3.5 \\
\hline B3LYP-D3/6-311G(2d,2p) & 4867.3 & 15.2 & 1636.1 & 4.9 & 1233.7 & 3.1 \\
\hline B3LYP-D3/6-311+G(2d,2p) & 4867.8 & 15.7 & 1634.5 & 3.3 & 1232.8 & 2.2 \\
\hline B3LYP-D3/6-311++G(2d,2p) & 4867.7 & 15.6 & 1634.5 & 3.3 & 1232.8 & 2.2 \\
\hline B3LYP-D3/6-311G(2df,2pd) & 4874.8 & 22.7 & 1638.5 & 7.3 & 1235.6 & 5.0 \\
\hline B3LYP-D3/6-311+G(2df,2pd) & 4875.6 & 23.5 & 1636.5 & 5.3 & 1234.5 & 3.9 \\
\hline B3LYP-D3/6-311++G(2df,2pd) & 4875.6 & 23.5 & 1636.5 & 5.3 & 1234.5 & 3.9 \\
\hline B3LYP-D3BJ/6-31G(d,p) & 4835.9 & -16.3 & 1628.6 & -2.7 & 1227.5 & -3.1 \\
\hline B3LYP-D3BJ/6-31+G(d,p) & 4835.2 & -17.0 & 1624.2 & -7.0 & 1225.0 & -5.6 \\
\hline B3LYP-D3BJ/6-31++G(d,p) & 4835.2 & -16.9 & 1624.2 & -7.1 & 1225.0 & -5.6 \\
\hline B3LYP-D3BJ/6-311G(d,p) & 4856.1 & 4.0 & 1631.7 & 0.5 & 1230.6 & 0.0 \\
\hline B3LYP-D3BJ/6-311+G(d,p) & 4856.8 & 4.7 & 1630.1 & -1.2 & 1229.7 & -0.9 \\
\hline B3LYP-D3BJ/6-311++G(d,p) & 4856.7 & 4.5 & 1630.1 & -1.1 & 1229.7 & -0.9 \\
\hline B3LYP-D3BJ/6-311G(df,pd) & 4870.4 & 18.2 & 1635.8 & 4.6 & 1233.8 & 3.2 \\
\hline B3LYP-D3BJ/6-311+G(df,pd) & 4871.5 & 19.4 & 1633.9 & 2.6 & 1232.8 & 2.2 \\
\hline B3LYP-D3BJ/6-311++G(df,pd) & 4871.4 & 19.2 & 1633.9 & 2.7 & 1232.8 & 2.2 \\
\hline B3LYP-D3BJ/6-311G(2d,2p) & 4871.3 & 19.2 & 1635.4 & 4.2 & 1233.6 & 3.0 \\
\hline B3LYP-D3BJ/6-311+G(2d,2p) & 4871.8 & 19.7 & 1633.8 & 2.6 & 1232.7 & 2.1 \\
\hline B3LYP-D3BJ/6-311++G(2d,2p) & 4871.7 & 19.6 & 1633.8 & 2.6 & 1232.7 & 2.1 \\
\hline B3LYP-D3BJ/6-311G(2df,2pd) & 4878.7 & 26.6 & 1637.9 & 6.7 & 1235.5 & 4.9 \\
\hline B3LYP-D3BJ/6-311+G(2df,2pd) & 4879.3 & 27.2 & 1635.7 & 4.5 & 1234.3 & 3.7 \\
\hline B3LYP-D3BJ/6-311++G(2df,2pd) & 4879.2 & 27.1 & 1635.7 & 4.5 & 1234.3 & 3.7 \\
\hline B3LYP-D3BJ/6-311G(3df,3pd) & 4882.6 & 30.5 & 1638.0 & 6.8 & 1235.8 & 5.2 \\
\hline B3LYP-D3BJ/6-311+G(3df,3pd) & 4881.0 & 28.8 & 1636.2 & 5.0 & 1234.7 & 4.1 \\
\hline B3LYP-D3BJ/6-311++G(3df,3pd) & 4881.0 & 28.8 & 1636.2 & 5.0 & 1234.7 & 4.1 \\
\hline B3LYP-D3BJ/cc-pVDZ & 4825.0 & -27.1 & 1626.7 & -4.6 & 1225.8 & -4.8 \\
\hline B3LYP-D3BJ/cc-pVTZ & 4876.8 & 24.7 & 1636.1 & 4.8 & 1234.3 & 3.7 \\
\hline B3LYP-D3BJ/aug-cc-pVDZ & 4831.7 & -20.5 & 1623.2 & -8.1 & 1224.2 & -6.3 \\
\hline B3LYP-D3BJ/aug-cc-pVTZ & 4877.1 & 25.0 & 1635.5 & 4.2 & 1234.0 & 3.4 \\
\hline CAM-B3LYP-D3BJ/6-311G(d,p) & 4901.7 & 49.6 & 1643.6 & 12.4 & 1240.3 & 9.7 \\
\hline CAM-B3LYP-D3BJ/6-311+G(d,p) & 4902.4 & 50.3 & 1642.0 & 10.8 & 1239.4 & 8.8 \\
\hline CAM-B3LYP-D3BJ/6-311++G(d,p) & 4902.2 & 50.1 & 1642.0 & 10.8 & 1239.4 & 8.8 \\
\hline CAM-B3LYP-D3BJ/cc-pVDZ & 4870.2 & 18.1 & 1638.5 & 7.3 & 1235.4 & 4.8 \\
\hline CAM-B3LYP-D3BJ/cc-pVTZ & 4921.7 & 69.6 & 1647.8 & 16.6 & 1243.9 & 13.3 \\
\hline CAM-B3LYP-D3BJ/aug-cc-pVDZ & 4876.7 & 24.6 & 1634.7 & 3.5 & 1233.7 & 3.1 \\
\hline CAM-B3LYP-D3BJ/aug-cc-pVTZ & 4922.2 & 70.1 & 1647.1 & 15.9 & 1243.5 & 12.9 \\
\hline M06-2X/6-31G(d,p) & 4872.6 & 20.4 & 1640.9 & 9.7 & 1236.9 & 6.3 \\
\hline M06-2X/6-31+G(d,p) & 4871.9 & 19.8 & 1637.0 & 5.7 & 1234.6 & 4.0 \\
\hline M06-2X/6-31++G(d,p) & 4872.0 & 19.9 & 1636.9 & 5.7 & 1234.6 & 4.0 \\
\hline
\end{tabular}




\begin{tabular}{|c|c|c|c|c|c|c|}
\hline M06-2X/6-311G(d,p) & 4889.0 & 36.9 & 1643.8 & 12.6 & 1239.6 & 9.0 \\
\hline M06-2X/6-311+G(d,p) & 4890.1 & 38.0 & 1642.3 & 11.0 & 1238.8 & 8.2 \\
\hline$M 06-2 X / 6-311++G(d, p)$ & 4889.9 & 37.8 & 1642.3 & 11.1 & 1238.8 & 8.2 \\
\hline M06-2X/6-311G(df,pd) & 4899.6 & 47.5 & 1646.9 & 15.7 & 1242.0 & 11.4 \\
\hline M06-2X/6-311+G(df,pd) & 4901.4 & 49.2 & 1645.2 & 13.9 & 1241.1 & 10.5 \\
\hline M06-2X/6-311++G(df,pd) & 4901.2 & 49.1 & 1645.2 & 14.0 & 1241.1 & 10.6 \\
\hline M06-2X/6-311G(3df,3pd) & 4912.2 & 60.1 & 1648.6 & 17.3 & 1243.7 & 13.1 \\
\hline M06-2X/6-311+G(3df,3pd) & 4910.9 & 58.7 & 1647.4 & 16.1 & 1243.0 & 12.4 \\
\hline M06-2X/6-311++G(3df,3pd) & 4910.9 & 58.7 & 1647.3 & 16.1 & 1242.9 & 12.3 \\
\hline M06-2X/cc-pVDZ & 4864.0 & 11.9 & 1640.0 & 8.8 & 1235.9 & 5.3 \\
\hline M06-2X/cc-pVTZ & 4906.7 & 54.5 & 1647.4 & 16.1 & 1242.7 & 12.1 \\
\hline M06-2X/aug-cc-pVDZ & 4868.4 & 16.3 & 1636.1 & 4.9 & 1234.0 & 3.4 \\
\hline M06-2X/aug-cc-pVTZ & 4907.8 & 55.7 & 1647.0 & 15.7 & 1242.5 & 11.9 \\
\hline WB97XD/6-311G(d,p) & 4893.0 & 40.9 & 1640.7 & 9.5 & 1238.1 & 7.5 \\
\hline WB97XD/6-311+G(d,p) & 4892.8 & 40.7 & 1640.7 & 9.5 & 1238.1 & 7.5 \\
\hline WB97XD/6-311++G(d,p) & 4891.8 & 39.7 & 1642.1 & 10.9 & 1238.8 & 8.2 \\
\hline WB97XD/cc-pVDZ & 4862.1 & 10.0 & 1636.3 & 5.1 & 1233.7 & 3.1 \\
\hline WB97XD/cc-pVTZ & 4911.8 & 59.7 & 1645.8 & 14.6 & 1242.1 & 11.5 \\
\hline WB97XD/aug-cc-pVDZ & 4867.0 & 14.9 & 1633.1 & 1.9 & 1232.2 & 1.6 \\
\hline WB97XD/aug-cc-pVTZ & 4912.5 & 60.4 & 1645.5 & 14.3 & 1242.0 & 11.4 \\
\hline CCSD/cc-pVDZ & 4797.2 & -54.9 & 1616.5 & -14.7 & 1218.4 & -12.2 \\
\hline B2PLYP-D3/6-311+G(3df,2pd) & 4872.71 & & 1638.66 & & 1235.53 & \\
\hline $\operatorname{CCSD}(T) \_a e / c c-p w C V T Z$ & 4867.45 & & 1639.53 & & 1235.73 & \\
\hline $\operatorname{CCSD}(\mathrm{T}) \_a e / c c-p w C V Q Z, X_{\mathrm{e}}^{\mathrm{BO} a}$ & 4881.49 & & 1642.35 & & 1238.22 & \\
\hline Experiment, $X_{0}$ & 4852.1 & & 1631.2 & & 1230.6 & \\
\hline Semiexperimental, $X_{\mathrm{e}}^{\mathrm{SE} \mathbf{b}}$ & 4881.99 & $0.01 \%^{c}$ & 1643.54 & $0.07 \%^{c}$ & 1238.49 & $0.02 \%^{c}$ \\
\hline Semiexperimental, $X_{\mathrm{e}}^{\mathrm{SE} d}$ & 4883.22 & $0.03 \%^{\mathrm{c}}$ & 1643.72 & $0.08 \%^{\mathrm{c}}$ & 1239.15 & $0.08 \%^{\mathrm{c}}$ \\
\hline
\end{tabular}

${ }^{a}$ Rotational constants for $r_{\mathrm{e}}^{\mathrm{BO}}$ structure, i.e. of $\operatorname{CCSD}(\mathrm{T}) \_$ae/cc-pwCVQZ quality.

${ }^{b}$ Derived with the MP2/6-311++G(d,p) anharmonic force field.

c Deviation from calculated equilibrium rotational constant of CCSD(T)_ae/cc-pwCVQZ quality.

${ }^{d}$ Derived with the B2PLYP-D3/6-311+G(3df,2pd) anharmonic force field. 
Table S3: Equilibrium rotational constants of syn-2AF calculated at various levels of theory. The deviations between the calculated and the experimental ground state values $X_{0}$ are given as $\Delta A$, $\Delta B$ and $\Delta C$.

\begin{tabular}{|c|c|c|c|c|c|c|}
\hline Method/Basis Set & $\begin{array}{c}\boldsymbol{A} / \\
\mathrm{MHz}\end{array}$ & $\begin{array}{l}\Delta \boldsymbol{A} / \\
\mathbf{M H z}\end{array}$ & $\begin{array}{c}B / \\
\mathrm{MHz}\end{array}$ & $\begin{array}{l}\Delta B / \\
\mathrm{MHz}\end{array}$ & $\begin{array}{c}C / \\
\mathrm{MHz}\end{array}$ & $\begin{array}{l}\Delta \boldsymbol{C} / \\
\mathrm{MHz}\end{array}$ \\
\hline MP2/6-31G(d,p) & 4864.9 & -37.6 & 1616.4 & 1.3 & 1222.5 & -2.2 \\
\hline $\mathrm{MP} 2 / 6-31+\mathrm{G}(\mathrm{d}, \mathrm{p})$ & 4857.1 & -45.3 & 1611.3 & -3.8 & 1219.1 & -5.6 \\
\hline MP2/6-31++G(d,p) & 4857.0 & -45.5 & 1611.3 & -3.8 & 1219.1 & -5.6 \\
\hline MP2/6-311G(d,p) & 4863.6 & -38.8 & 1618.3 & 3.2 & 1223.6 & -1.1 \\
\hline $\mathrm{MP} 2 / 6-311+\mathrm{G}(\mathrm{d}, \mathrm{p})$ & 4859.5 & -42.9 & 1616.4 & 1.3 & 1222.2 & -2.4 \\
\hline MP2/6-311++G(d,p) & 4859.5 & -42.9 & 1616.4 & 1.3 & 1222.2 & -2.4 \\
\hline MP2/6-311G(df,pd) & 4893.1 & -9.4 & 1628.5 & 13.3 & 1231.2 & 6.5 \\
\hline MP2/6-311+G(df,pd) & 4892.8 & -9.7 & 1625.7 & 10.6 & 1229.6 & 4.9 \\
\hline MP2/6-311++G(df,pd) & 4892.3 & -10.1 & 1625.8 & 10.7 & 1229.6 & 4.9 \\
\hline MP2/6-311G(2d,2p) & 4879.9 & -22.5 & 1622.6 & 7.5 & 1226.9 & 2.2 \\
\hline MP2/6-311+G(2d,2p) & 4878.8 & -23.6 & 1621.0 & 5.9 & 1226.0 & 1.3 \\
\hline MP2/6-311++G(2d,2p) & 4878.6 & -23.8 & 1621.1 & 6.0 & 1226.0 & 1.3 \\
\hline MP2/6-311G(2df,2pd) & 4901.8 & -0.6 & 1632.4 & 17.3 & 1233.9 & 9.2 \\
\hline MP2/6-311+G(2df,2pd) & 4899.2 & -3.2 & 1630.3 & 15.2 & 1232.5 & 7.8 \\
\hline MP2/6-311++G(2df,2pd) & 4899.0 & -3.4 & 1630.3 & 15.2 & 1232.5 & 7.8 \\
\hline MP2/6-311G(3df,3pd) & 4905.4 & 2.9 & 1629.5 & 14.3 & 1232.5 & 7.8 \\
\hline MP2/6-311+G(3df,3pd) & 4899.0 & -3.5 & 1628.4 & 13.3 & 1231.5 & 6.8 \\
\hline MP2/6-311++G(3df,3pd) & 4898.8 & -3.6 & 1628.5 & 13.3 & 1231.5 & 6.8 \\
\hline MP2/cc-pVDZ & 4815.7 & -86.8 & 1608.1 & -7.0 & 1214.8 & -9.9 \\
\hline MP2/cc-pVTZ & 4889.5 & -12.9 & 1628.3 & 13.2 & 1230.8 & 6.1 \\
\hline MP2/aug-cc-pVDZ & 4804.4 & -98.0 & 1600.4 & -14.8 & 1209.7 & -15.0 \\
\hline MP2/aug-cc-pVTZ & 4888.1 & -14.3 & 1627.0 & 11.9 & 1230.0 & 5.3 \\
\hline B3LYP-D3/6-31G(d,p) & 4879.4 & -23.0 & 1611.8 & -3.3 & 1220.8 & -3.9 \\
\hline B3LYP-D3/6-31+G(d,p) & 4879.2 & -23.2 & 1607.3 & -7.8 & 1218.2 & -6.5 \\
\hline B3LYP-D3/6-31++G(d,p) & 4879.3 & -23.1 & 1607.3 & -7.8 & 1218.2 & -6.5 \\
\hline B3LYP-D3/6-311G(d,p) & 4900.9 & -1.5 & 1614.6 & -0.5 & 1223.7 & -1.0 \\
\hline B3LYP-D3/6-311+G(d,p) & 4902.3 & -0.1 & 1612.8 & -2.3 & 1222.8 & -1.9 \\
\hline B3LYP-D3/6-311++G(d,p) & 4902.1 & -0.3 & 1612.8 & -2.3 & 1222.8 & -1.9 \\
\hline B3LYP-D3/6-311G(df,pd) & 4914.6 & 12.2 & 1618.4 & 3.3 & 1226.7 & 2.0 \\
\hline B3LYP-D3/6-311+G(df,pd) & 4916.5 & 14.1 & 1616.5 & 1.4 & 1225.8 & 1.1 \\
\hline B3LYP-D3/6-311++G(df,pd) & 4916.3 & 13.9 & 1616.6 & 1.5 & 1225.8 & 1.1 \\
\hline B3LYP-D3/6-311G(2d,2p) & 4917.7 & 15.3 & 1618.1 & 3.0 & 1226.7 & 2.0 \\
\hline B3LYP-D3/6-311+G(2d,2p) & 4917.2 & 14.8 & 1617.0 & 1.9 & 1226.1 & 1.4 \\
\hline B3LYP-D3/6-311++G(2d,2p) & 4917.1 & 14.7 & 1617.1 & 2.0 & 1226.1 & 1.4 \\
\hline B3LYP-D3/6-311G(2df,2pd) & 4924.9 & 22.5 & 1620.2 & 5.1 & 1228.4 & 3.7 \\
\hline B3LYP-D3/6-311+G(2df,2pd) & 4924.8 & 22.4 & 1618.7 & 3.6 & 1227.5 & 2.8 \\
\hline
\end{tabular}




\begin{tabular}{|c|c|c|c|c|c|c|}
\hline B3LYP-D3/6-311++G(2df,2pd) & 4924.7 & 22.3 & 1618.8 & 3.7 & 1227.5 & 2.8 \\
\hline B3LYP-D3/6-311G(3df,3pd) & 4929.5 & 27.1 & 1619.8 & 4.7 & 1228.4 & 3.7 \\
\hline B3LYP-D3/6-311+G(3df,3pd) & 4925.4 & 23.0 & 1619.2 & 4.1 & 1227.8 & 3.1 \\
\hline B3LYP-D3/6-311++G(3df,3pd) & 4925.4 & 23.0 & 1619.2 & 4.1 & 1227.8 & 3.1 \\
\hline B3LYP-D3/cc-pVDZ & 4866.4 & -36.0 & 1610.6 & -4.5 & 1219.4 & -5.3 \\
\hline B3LYP-D3/cc-pVTZ & 4921.5 & 19.1 & 1619.2 & 4.1 & 1227.6 & 2.9 \\
\hline B3LYP-D3/aug-cc-pVDZ & 4873.9 & -28.5 & 1606.7 & -8.4 & 1217.6 & -7.1 \\
\hline B3LYP-D3/aug-cc-pVTZ & 4922.1 & 19.7 & 1618.7 & 3.6 & 1227.3 & 2.6 \\
\hline B3LYP-D3BJ/6-31G(d,p) & 4884.0 & -18.4 & 1612.4 & -2.7 & 1221.4 & -3.3 \\
\hline B3LYP-D3BJ/6-31+G(d,p) & 4883.9 & -18.5 & 1607.8 & -7.3 & 1218.8 & -5.8 \\
\hline B3LYP-D3BJ/6-31++G(d,p) & 4883.9 & -18.5 & 1607.8 & -7.3 & 1218.8 & -5.9 \\
\hline B3LYP-D3BJ/6-311G(d,p) & 4905.5 & 3.1 & 1615.1 & 0.0 & 1224.3 & -0.4 \\
\hline B3LYP-D3BJ/6-311+G(d,p) & 4906.7 & 4.2 & 1613.3 & -1.8 & 1223.3 & -1.3 \\
\hline B3LYP-D3BJ/6-311++G(d,p) & 4906.5 & 4.1 & 1613.3 & -1.8 & 1223.3 & -1.3 \\
\hline B3LYP-D3BJ/6-311G(df,pd) & 4919.2 & 16.8 & 1618.8 & 3.7 & 1227.3 & 2.6 \\
\hline B3LYP-D3BJ/6-311+G(df,pd) & 4920.8 & 18.4 & 1617.0 & 1.9 & 1226.3 & 1.7 \\
\hline B3LYP-D3BJ/6-311++G(df,pd) & 4920.6 & 18.2 & 1617.0 & 1.9 & 1226.3 & 1.7 \\
\hline B3LYP-D3BJ/6-311G(2d,2p) & 4922.4 & 20.0 & 1618.5 & 3.4 & 1227.3 & 2.6 \\
\hline B3LYP-D3BJ/6-311+G(2d,2p) & 4921.6 & 19.2 & 1617.5 & 2.4 & 1226.6 & 1.9 \\
\hline B3LYP-D3BJ/6-311++G(2d,2p) & 4921.5 & 19.1 & 1617.5 & 2.4 & 1226.6 & 1.9 \\
\hline B3LYP-D3BJ/6-311G(2df,2pd) & 4929.5 & 27.1 & 1620.6 & 5.5 & 1228.9 & 4.2 \\
\hline B3LYP-D3BJ/6-311+G(2df,2pd) & 4929.1 & 26.7 & 1619.1 & 4.0 & 1228.0 & 3.3 \\
\hline B3LYP-D3BJ/6-311++G(2df,2pd) & 4929.1 & 26.7 & 1619.2 & 4.1 & 1228.0 & 3.3 \\
\hline B3LYP-D3BJ/6-311G(3df,3pd) & 4933.7 & 31.3 & 1620.0 & 4.9 & 1228.8 & 4.1 \\
\hline B3LYP-D3BJ/6-311+G(3df,3pd) & 4929.7 & 27.2 & 1619.6 & 4.5 & 1228.3 & 3.7 \\
\hline B3LYP-D3BJ/6-311++G(3df,3pd) & 4929.6 & 27.2 & 1619.6 & 4.5 & 1228.3 & 3.7 \\
\hline B3LYP-D3BJ/cc-pVDZ & 4871.0 & -31.4 & 1611.0 & -4.1 & 1219.9 & -4.7 \\
\hline B3LYP-D3BJ/cc-pVTZ & 4926.0 & 23.5 & 1619.6 & 4.5 & 1228.1 & 3.5 \\
\hline B3LYP-D3BJ/aug & 4878.5 & -23.9 & 1607.4 & -7.8 & 1218.3 & -6.4 \\
\hline B3LYP-D3BJ/aug & 4926.2 & 23.8 & 1618.9 & 3.8 & 1227.7 & 3.1 \\
\hline CAM-B3LYP-D3BJ/6-311G(d,p) & 4955.6 & 53.2 & 1626.0 & 10.9 & 1233.7 & 9.0 \\
\hline CAM-B3LYP-D3E & 4956.9 & 54.5 & 1624.1 & 9.0 & 1232.7 & 8.0 \\
\hline CAM-B3LYP-D3BJ/6-311++G(d,p) & 4956.7 & 54.3 & 1624.1 & 9.0 & 1232.7 & 8.0 \\
\hline CAM-B3LYP-D3BJ/cc-pVDZ & 4921.6 & 19.2 & 1621.6 & 6.5 & 1229.1 & 4.4 \\
\hline CAM-B3LYP-D3BJ/cc-pVTZ & 4975.0 & 72.6 & 1630.5 & 15.4 & 1237.4 & 12.7 \\
\hline CAM-B3LYP-D3BJ/aug-cc-pVDZ & 4928.3 & 25.9 & 1617.5 & 2.4 & 1227.2 & 2.5 \\
\hline CAM-B3LYP-D3BJ/aug-cc-pVTZ & 4975.4 & 73.0 & 1629.8 & 14.7 & 1237.0 & 12.3 \\
\hline M06-2X/6-31G(d,p) & 4922.7 & 20.3 & 1623.7 & 8.6 & 1230.3 & 5.7 \\
\hline M06-2X/6-31+G(d,p) & 4923.0 & 20.6 & 1619.8 & 4.7 & 1228.2 & 3.5 \\
\hline M06-2X/6-31++G(d,p) & 4922.9 & 20.5 & 1619.8 & 4.7 & 1228.2 & 3.5 \\
\hline M06-2X/6-311G(d,p) & 4938.8 & 36.4 & 1626.6 & 11.5 & 1233.0 & 8.3 \\
\hline M06-2X/6-311+G(d,p) & 4940.1 & 37.6 & 1625.2 & 10.1 & 1232.3 & 7.6 \\
\hline M06-2X/6-311++G(d,p) & 4939.8 & 37.4 & 1625.3 & 10.2 & 1232.3 & 7.6 \\
\hline
\end{tabular}




\begin{tabular}{lrrrrrr}
\hline M06-2X/6-311G(df,pd) & 4948.5 & 46.1 & 1629.5 & 14.4 & 1235.2 & 10.6 \\
M06-2X/6-311+G(df,pd) & 4950.8 & 48.3 & 1627.9 & 12.8 & 1234.5 & 9.8 \\
M06-2X/6-311++G(df,pd) & 4950.6 & 48.1 & 1628.0 & 12.9 & 1234.5 & 9.8 \\
M06-2X/6-311G(3df,3pd) & 4963.2 & 60.8 & 1630.7 & 15.6 & 1236.8 & 12.2 \\
M06-2X/6-311+G(3df,3pd) & 4959.5 & 57.0 & 1630.5 & 15.4 & 1236.5 & 11.8 \\
M06-2X/6-311++G(3df,3pd) & 4959.4 & 57.0 & 1630.6 & 15.4 & 1236.5 & 11.8 \\
M06-2X/cc-pVDZ & 4911.9 & 9.5 & 1623.6 & 8.5 & 1229.7 & 5.0 \\
M06-2X/cc-pVTZ & 4954.7 & 52.3 & 1630.8 & 15.7 & 1236.3 & 11.7 \\
M06-2X/aug-cc-pVDZ & 4916.5 & 14.0 & 1619.9 & 4.8 & 1227.9 & 3.2 \\
M06-2X/aug-cc-pVTZ & 4955.8 & 53.4 & 1630.4 & 15.3 & 1236.2 & 11.5 \\
WB97XD/6-311G(d,p) & 4941.4 & 39.0 & 1624.8 & 9.7 & 1232.1 & 7.4 \\
WB97XD/6-311+G(d,p) & 4941.2 & 38.8 & 1624.8 & 9.7 & 1232.1 & 7.4 \\
WB97XD/6-311++G(d,p) & 4939.5 & 37.1 & 1626.3 & 11.2 & 1232.8 & 8.1 \\
WB97XD/cc-pVDZ & 4908.0 & 5.6 & 1621.4 & 6.3 & 1228.2 & 3.5 \\
WB97XD/cc-pVTZ & 4958.5 & 56.1 & 1630.5 & 15.4 & 1236.4 & 11.7 \\
WB97XD/aug-cc-pVDZ & 4913.0 & 10.6 & 1618.3 & 3.2 & 1226.7 & 2.0 \\
WB97XD/aug-cc-pVTZ & 4959.0 & 56.6 & 1630.3 & 15.2 & 1236.3 & 11.6 \\
CCSD/cc-pVDZ & 4844.3 & -58.1 & 1600.8 & -14.3 & 1212.5 & -12.2 \\
\hline B2PLYP-D3/6-311+G(3df,2pd) & 4920.36 & & 1622.03 & & 1229.14 & \\
CCSD(T)_ae/cc-pwCVTZ & 4909.45 & & 1627.12 & & 1231.39 & \\
CCSD(T)_ae/cc-pwCVQZ, Xe a & 4927.85 & & $\mathbf{1 6 2 6 . 5 5}$ & & $\mathbf{1 2 3 2 . 2 2}$ & \\
\hline Experiment, X & $\mathbf{4 9 0 2 . 4}$ & & $\mathbf{1 6 1 5 . 1}$ & & $\mathbf{1 2 2 4 . 7}$ & \\
\hline
\end{tabular}

a Rotational constants for $r_{\mathrm{e}}^{\mathrm{BO}}$ structure, i.e. of $\operatorname{CCSD}(\mathrm{T})$ _ae/cc-pwCVQZ quality. 
Table S4a: Born-Oppenheimer $r_{\mathrm{e}}^{\mathrm{BO}}$ structures of anti-2AF (bond lengths in $\AA$, angles in degree). Comparison with results of other calculations is given.

\begin{tabular}{|c|c|c|c|c|c|c|}
\hline $\begin{array}{l}\text { Method } \\
\text { Basis set }\end{array}$ & $\begin{array}{c}\text { B2PLYP-D3 } \\
6-311+G(3 d f, 2 p d)\end{array}$ & $\begin{array}{l}\text { MP2_fc } \\
\text { cc-pVTZ }\end{array}$ & $\begin{array}{l}\text { MP2_ae } \\
\text { cc-pwCVTZ }\end{array}$ & $\begin{array}{l}\text { MP2_ae } \\
\text { cc-pwCVQZ }\end{array}$ & $\begin{array}{l}\text { CCSD(T)_ae } \\
\text { cc-pwCVTZ }\end{array}$ & $\begin{array}{l}\text { anti } \\
r_{\mathrm{e}}^{\mathrm{BO}}\end{array}$ \\
\hline $\mathrm{O} 1-\mathrm{C} 2$ & 1.3707 & 1.3643 & 1.3607 & 1.3587 & 1.3680 & 1.3660 \\
\hline $\mathrm{C} 2=\mathrm{C} 3$ & 1.3672 & 1.3749 & 1.3702 & 1.3689 & 1.3647 & 1.3634 \\
\hline $\mathrm{C} 4=\mathrm{C} 5$ & 1.3631 & 1.3697 & 1.3650 & 1.3635 & 1.3620 & 1.3605 \\
\hline C5-O1 & 1.3517 & 1.3544 & 1.3508 & 1.3483 & 1.3547 & 1.3522 \\
\hline C2-C6 & 1.4656 & 1.4665 & 1.4621 & 1.4608 & 1.4707 & 1.4694 \\
\hline $\mathrm{C} 6=\mathrm{O} 7$ & 1.2184 & 1.2227 & 1.2193 & 1.2179 & 1.2168 & 1.2154 \\
\hline C6-C8 & 1.5079 & 1.5054 & 1.5009 & 1.4986 & 1.5075 & 1.5051 \\
\hline C3-H11 & 1.0746 & 1.0754 & 1.0741 & 1.0734 & 1.0757 & 1.0750 \\
\hline $\mathrm{C} 4-\mathrm{H} 10$ & 1.0748 & 1.0756 & 1.0742 & 1.0734 & 1.0759 & 1.0751 \\
\hline C5-H9 & 1.0741 & 1.0749 & 1.0735 & 1.0727 & 1.0752 & 1.0744 \\
\hline $\mathrm{C} 8-\mathrm{H}_{\mathrm{ip}} \mathrm{b}$ & 1.0847 & 1.0850 & 1.0831 & 1.0822 & 1.0859 & 1.0850 \\
\hline $\mathrm{C} 8-\mathrm{H}_{\mathrm{op}}^{\mathrm{b}}$ & 1.0894 & 1.0892 & 1.0872 & 1.0864 & 1.0903 & 1.0895 \\
\hline $\mathrm{O} 1-\mathrm{C} 2=\mathrm{C} 3$ & 109.615 & 110.004 & 109.990 & 109.920 & 110.160 & 110.090 \\
\hline C2-O1-C5 & 107.014 & 106.853 & 106.838 & 106.956 & 106.679 & 106.796 \\
\hline $\mathrm{O} 1-\mathrm{C} 5=\mathrm{C} 4$ & 110.684 & 110.641 & 110.629 & 110.562 & 110.920 & 110.854 \\
\hline $\mathrm{C} 2-\mathrm{C} 6=\mathrm{O} 7$ & 119.934 & 119.934 & 119.915 & 119.885 & 119.874 & 119.844 \\
\hline C2-C6-C8 & 117.319 & 116.984 & 117.015 & 117.118 & 117.082 & 117.185 \\
\hline O1-C2-C6 & 118.468 & 118.279 & 118.379 & 118.304 & 118.394 & 118.319 \\
\hline $\mathrm{O} 1-\mathrm{C} 5-\mathrm{H} 9$ & 116.056 & 115.932 & 115.997 & 116.040 & 115.977 & 116.020 \\
\hline $\mathrm{C} 5=\mathrm{C} 4-\mathrm{H} 10$ & 126.216 & 125.881 & 125.887 & 125.872 & 126.294 & 126.279 \\
\hline C4-C3-H11 & 128.347 & 128.798 & 128.801 & 128.791 & 128.503 & 128.493 \\
\hline $\mathrm{C} 6-\mathrm{C} 8-\mathrm{H}_{\mathrm{ip}}{ }^{\mathrm{b}}$ & 109.027 & 108.976 & 108.977 & 108.993 & 108.983 & 109.000 \\
\hline $\mathrm{C} 6-\mathrm{C} 8-\mathrm{H}_{\mathrm{op}}^{\mathrm{b}}$ & 110.287 & 110.170 & 110.196 & 110.186 & 110.123 & 110.113 \\
\hline $\mathrm{Hop}_{\mathrm{op}}-\mathrm{C} 8-\mathrm{C} 6-\mathrm{C} 2^{\mathrm{b}}$ & \pm 58.954 & \pm 58.957 & \pm 58.959 & \pm 58.937 & \pm 58.945 & \pm 58.923 \\
\hline
\end{tabular}

a Frozen core, only valence electrons included.

${ }^{b} \mathrm{H}_{\text {ip }}$ is the in-plane hydrogen atom (H13 in Figure 1$)$ and $\mathrm{H}_{\mathrm{op}}$ the two out-of-plane ones $(\mathrm{H} 12$ and 14 in Figure 1). 
Table S4b: Born-Oppenheimer $r_{\mathrm{e}}^{\mathrm{BO}}$ structures of syn-2AF (bond lengths in $\AA$, angles in degree). Comparison with results of other calculations is given.

\begin{tabular}{|c|c|c|c|c|}
\hline $\begin{array}{l}\text { Method } \\
\text { Basis set }\end{array}$ & $\begin{array}{l}\text { MP2_ae } \\
\text { cc-pwCVTZ }\end{array}$ & $\begin{array}{l}\text { MP2_ae } \\
\text { cc-pwCVQZ }\end{array}$ & $\begin{array}{l}\text { CCSD(T)_ae } \\
\text { cc-pwCVTZ }\end{array}$ & $\begin{array}{l}\text { syn } \\
r_{\mathrm{e}}^{\mathrm{BO}}\end{array}$ \\
\hline C5-O1 & 1.3476 & 1.3453 & 1.3515 & 1.3492 \\
\hline C2-O1 & 1.3554 & 1.3527 & 1.3616 & 1.3589 \\
\hline C2-O1-C5 & 106.983 & 107.114 & 106.856 & 106.987 \\
\hline C4-C5 & 1.3646 & 1.3631 & 1.3618 & 1.3603 \\
\hline O1-C5-C4 & 110.919 & 110.841 & 111.175 & 111.096 \\
\hline C2-C3 & 1.3735 & 1.3723 & 1.3678 & 1.3666 \\
\hline O1-C2-C3 & 109.827 & 109.772 & 110.014 & 109.959 \\
\hline C5-H9 & 1.0736 & 1.0728 & 1.0752 & 1.0744 \\
\hline O1-C5-H9 & 115.904 & 115.940 & 115.890 & 115.926 \\
\hline C2-C6 & 1.4628 & 1.4615 & 1.4717 & 1.4703 \\
\hline O1-C2-C6 & 117.609 & 117.731 & 117.639 & 117.761 \\
\hline $\mathrm{C} 4-\mathrm{H} 10$ & 1.0741 & 1.0733 & 1.0757 & 1.0750 \\
\hline C5-C4-H10 & 126.181 & 126.158 & 126.586 & 126.563 \\
\hline C3-H11 & 1.0745 & 1.0738 & 1.0763 & 1.0757 \\
\hline C4-C3-H11 & 127.628 & 127.649 & 127.417 & 127.438 \\
\hline $\mathrm{C} 6=\mathrm{O} 7$ & 1.2177 & 1.2162 & 1.2147 & 1.2132 \\
\hline $\mathrm{C} 2-\mathrm{C} 6=\mathrm{O} 7$ & 121.636 & 121.642 & 121.579 & 121.585 \\
\hline C6-C8 & 1.5045 & 1.5023 & 1.5111 & 1.5090 \\
\hline C2-C6-C8 & 115.597 & 115.699 & 115.734 & 115.836 \\
\hline $\mathrm{C} 8-\mathrm{H}_{\mathrm{ip}}^{\mathrm{b}}$ & 1.0832 & 1.0823 & 1.0859 & 1.0851 \\
\hline C6-C8- $\mathrm{H}_{\mathrm{ip}}{ }^{\mathrm{b}}$ & 108.844 & 108.844 & 108.811 & 108.811 \\
\hline $\mathrm{C} 8-\mathrm{H}_{\mathrm{op}}^{\mathrm{b}}$ & 1.0874 & 1.0866 & 1.0905 & 1.0897 \\
\hline C6-C8- $-H_{o p}^{b}$ & 110.387 & 110.379 & 110.366 & 110.358 \\
\hline $\mathrm{H}_{\mathrm{op}}-\mathrm{C} 8-\mathrm{C} 6-\mathrm{C} 2^{\mathrm{b}}$ & \pm 59.510 & \pm 59.509 & \pm 59.522 & \pm 59.521 \\
\hline
\end{tabular}




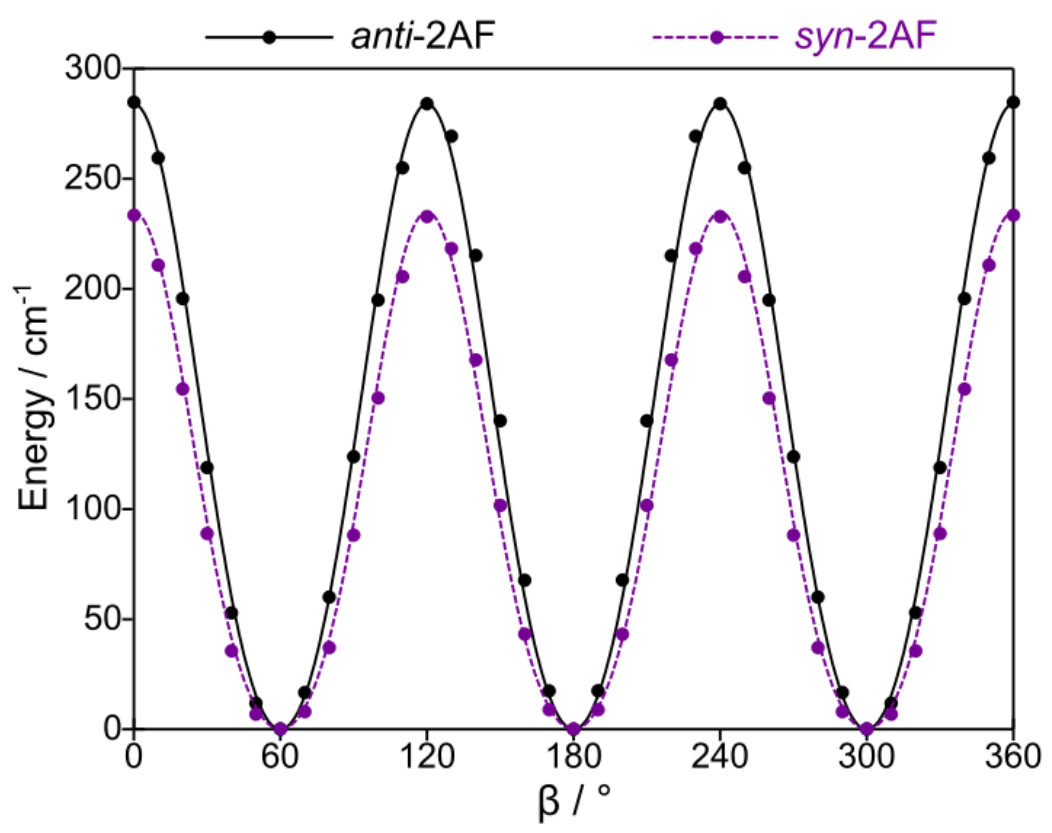

Figure S1: Potential energy curves of anti- and syn-2AF depending on the rotation of the methyl group about the $\mathrm{C} 6-\mathrm{C} 8$ bond upon variation of $\beta$. Calculations were carried out at the MP2/6$311++G(d, p)$ level of theory. The predicted $V_{3}$ potentials are $284.0 \mathrm{~cm}^{-1}$ and $234.8 \mathrm{~cm}^{-1}$ for antiand syn-2AF, respectively.

Coefficients of the one-dimensional Fourier expansion for the potential energy curves of anti- and syn-2AF given in Figure $S 1$ calculated at the MP2/6-311++G(d,p) level of theory. The potential is expanded as $V(\varphi)=\sum_{i=0}^{2} a_{i} f_{i}$.

\begin{tabular}{llrrrr}
\hline & \multicolumn{2}{c}{ anti-2AF } & \multicolumn{2}{c}{ syn-2AF } \\
$\boldsymbol{i} \mathbf{f}_{\boldsymbol{i}}$ & $\mathbf{a}_{\boldsymbol{i}} /$ Hartree & $\mathbf{a}_{\boldsymbol{i}} / \mathbf{c m}^{-\mathbf{1}}$ & $\mathbf{a}_{\boldsymbol{i}} /$ Hartree & $\mathbf{a}_{\boldsymbol{i}} / \mathbf{~ c m}^{-\mathbf{1}}$ \\
\hline 0 & 1 & -381.725125 & \multicolumn{3}{c}{-381.7233774} \\
1 & $\cos 3 \alpha$ & 0.0006468 & 142.0 & 0.0005350 & 117.4 \\
2 & $\cos 6 \alpha$ & 0.0000331 & 7.3 & 0.0000535 & 11.7 \\
\hline
\end{tabular}


Table S5: Observed frequencies $v_{o b s}$ of 293 rotational transitions of anti-2AF with $v_{o b s}-v_{c a l c}$ values obtained from a fit with the program XIAM.

\begin{tabular}{|c|c|c|c|c|c|c|c|c|c|}
\hline \multirow[t]{2}{*}{ No. } & $J$ & $K_{a}$ & $K_{c}$ & $J$ & $K_{a}$ & $K_{c}$ & \multirow[t]{2}{*}{ Species } & \multirow{2}{*}{$\begin{array}{c}\text { Vobs } \\
{[\mathrm{MHz}]}\end{array}$} & \multirow{2}{*}{$\begin{array}{c}\mathrm{V}_{\text {obs }}-\mathrm{V}_{\text {calc }} \\
{[\mathrm{kHz}]}\end{array}$} \\
\hline & \multicolumn{3}{|c|}{ upper level } & \multicolumn{3}{|c|}{ lower level } & & & \\
\hline 1 & 2 & 0 & 2 & 1 & 0 & 1 & $A$ & 5688.7409 & 0.9 \\
\hline 2 & 2 & 0 & 2 & 1 & 0 & 1 & $E$ & 5688.4292 & -1.9 \\
\hline 3 & 3 & 0 & 3 & 2 & 0 & 2 & $A$ & 8447.0826 & 0.1 \\
\hline 4 & 3 & 0 & 3 & 2 & 0 & 2 & $E$ & 8446.6958 & -0.7 \\
\hline 5 & 4 & 0 & 4 & 3 & 0 & 3 & $A$ & 11111.6951 & -0.5 \\
\hline 6 & 4 & 0 & 4 & 3 & 0 & 3 & $E$ & 11111.2984 & -0.8 \\
\hline 7 & 5 & 0 & 5 & 4 & 0 & 4 & $A$ & 13676.3249 & 0.3 \\
\hline 8 & 5 & 0 & 5 & 4 & 0 & 4 & $E$ & 13675.9669 & 0.0 \\
\hline 9 & 6 & 0 & 6 & 5 & 0 & 5 & $A$ & 16159.7298 & 0.0 \\
\hline 10 & 6 & 0 & 6 & 5 & 0 & 5 & $E$ & 16159.4275 & 0.4 \\
\hline 11 & 7 & 0 & 7 & 6 & 0 & 6 & $A$ & 18596.1692 & -0.2 \\
\hline 12 & 7 & 0 & 7 & 6 & 0 & 6 & $E$ & 18595.9112 & 0.5 \\
\hline 13 & 8 & 0 & 8 & 7 & 0 & 7 & $A$ & 21017.0136 & -1.0 \\
\hline 14 & 8 & 0 & 8 & 7 & 0 & 7 & $E$ & 21016.7814 & 0.5 \\
\hline 15 & 9 & 0 & 9 & 8 & 0 & 8 & $A$ & 23440.4036 & 7.5 \\
\hline 16 & 9 & 0 & 9 & 8 & 0 & 8 & $E$ & 23440.1724 & -0.4 \\
\hline 17 & 3 & 0 & 3 & 2 & 1 & 2 & $E$ & 5591.5659 & -2.7 \\
\hline 18 & 4 & 0 & 4 & 3 & 1 & 3 & $A$ & 8739.7131 & -0.7 \\
\hline 19 & 4 & 0 & 4 & 3 & 1 & 3 & $E$ & 8739.3590 & -2.6 \\
\hline 20 & 5 & 0 & 5 & 4 & 1 & 4 & $A$ & 11834.6106 & 0.2 \\
\hline 21 & 5 & 0 & 5 & 4 & 1 & 4 & $E$ & 11834.1079 & -2.5 \\
\hline 22 & 6 & 0 & 6 & 5 & 1 & 5 & $A$ & 14821.3088 & 0.3 \\
\hline 23 & 6 & 0 & 6 & 5 & 1 & 5 & $E$ & 14820.7629 & -1.5 \\
\hline 24 & 7 & 0 & 7 & 6 & 1 & 6 & $A$ & 17679.8733 & -0.2 \\
\hline 25 & 7 & 0 & 7 & 6 & 1 & 6 & $E$ & 17679.3498 & -1.4 \\
\hline 26 & 8 & 0 & 8 & 7 & 1 & 7 & $A$ & 20420.0777 & -0.8 \\
\hline 27 & 8 & 0 & 8 & 7 & 1 & 7 & $E$ & 20419.6077 & -0.8 \\
\hline 28 & 9 & 0 & 9 & 8 & 1 & 8 & $A$ & 23066.3704 & -1.8 \\
\hline 29 & 9 & 0 & 9 & 8 & 1 & 8 & $E$ & 23065.9598 & -1.3 \\
\hline 30 & 1 & 1 & 1 & 0 & 0 & 0 & $A$ & 6083.0065 & -2.0 \\
\hline 31 & 1 & 1 & 1 & 0 & 0 & 0 & $E$ & 6081.9240 & -0.1 \\
\hline 32 & 3 & 1 & 3 & 2 & 0 & 2 & $A$ & 10819.0609 & -3.5 \\
\hline 33 & 3 & 1 & 3 & 2 & 0 & 2 & $E$ & 10818.6356 & 1.4 \\
\hline 34 & 4 & 1 & 4 & 3 & 0 & 3 & $A$ & 12953.4055 & -4.3 \\
\hline 35 & 4 & 1 & 4 & 3 & 0 & 3 & $E$ & 12953.1585 & 2.8 \\
\hline 36 & 5 & 1 & 5 & 4 & 0 & 4 & $A$ & 15014.7425 & -3.4 \\
\hline 37 & 5 & 1 & 5 & 4 & 0 & 4 & $E$ & 15014.6323 & 2.7 \\
\hline 38 & 6 & 1 & 6 & 5 & 0 & 5 & A & 17076.0219 & -3.8 \\
\hline
\end{tabular}




\begin{tabular}{|c|c|c|c|c|c|c|c|c|c|}
\hline 39 & 6 & 1 & 6 & 5 & 0 & 5 & $E$ & 17075.9899 & 3.2 \\
\hline 40 & 7 & 1 & 7 & 6 & 0 & 6 & $\mathrm{E}$ & 19193.0861 & 3.0 \\
\hline 41 & 8 & 1 & 8 & 7 & 0 & 7 & A & 21391.0365 & -2.0 \\
\hline 42 & 8 & 1 & 8 & 7 & 0 & 7 & $\mathrm{E}$ & 21390.9943 & 1.8 \\
\hline 43 & 9 & 1 & 9 & 8 & 0 & 8 & A & 23667.8451 & -1.8 \\
\hline 44 & 9 & 1 & 9 & 8 & 0 & 8 & E & 23667.7619 & 0.5 \\
\hline 45 & 1 & 1 & 0 & 1 & 0 & 1 & A & 3621.8206 & 1.1 \\
\hline 46 & 1 & 1 & 0 & 1 & 0 & 1 & E & 3622.0541 & 1.1 \\
\hline 47 & 4 & 1 & 3 & 4 & 0 & 4 & A & 5835.6214 & 7.1 \\
\hline 48 & 4 & 1 & 3 & 4 & 0 & 4 & E & 5834.4467 & 4.0 \\
\hline 49 & 5 & 1 & 4 & 5 & 0 & 5 & A & 7296.0487 & -8.1 \\
\hline 50 & 5 & 1 & 4 & 5 & 0 & 5 & E & 7294.1788 & 2.6 \\
\hline 51 & 6 & 1 & 5 & 6 & 0 & 6 & A & 9173.9067 & -6.7 \\
\hline 52 & 6 & 1 & 5 & 6 & 0 & 6 & E & 9171.1505 & 0.7 \\
\hline 53 & 7 & 1 & 6 & 7 & 0 & 7 & A & 11432.5064 & -5.4 \\
\hline 54 & 7 & 1 & 6 & 7 & 0 & 7 & E & 11428.7760 & -1.8 \\
\hline 55 & 8 & 1 & 7 & 8 & 0 & 8 & A & 13984.8812 & -3.0 \\
\hline 56 & 8 & 1 & 7 & 8 & 0 & 8 & $\mathrm{E}$ & 13980.1941 & -2.0 \\
\hline 57 & 9 & 1 & 8 & 9 & 0 & 9 & A & 16716.5745 & 0.3 \\
\hline 58 & 9 & 1 & 8 & 9 & 0 & 9 & E & 16711.0269 & -1.2 \\
\hline 59 & 10 & 1 & 9 & 10 & 0 & 10 & A & 19515.6696 & 3.9 \\
\hline 60 & 10 & 1 & 9 & 10 & 0 & 10 & E & 19509.3911 & 1.8 \\
\hline 61 & 2 & 1 & 2 & 1 & 0 & 1 & A & 8544.1923 & -3.6 \\
\hline 62 & 2 & 1 & 2 & 1 & 0 & 1 & E & 8543.5597 & 0.7 \\
\hline 63 & 2 & 1 & 1 & 1 & 1 & 0 & A & 6124.5905 & 1.5 \\
\hline 64 & 2 & 1 & 1 & 1 & 1 & 0 & E & 6123.7435 & 0.4 \\
\hline 65 & 3 & 1 & 2 & 2 & 1 & 1 & A & 9163.8869 & 0.6 \\
\hline 66 & 3 & 1 & 2 & 2 & 1 & 1 & E & 9163.1381 & 0.8 \\
\hline 67 & 4 & 1 & 3 & 3 & 1 & 2 & A & 12172.8377 & 0.1 \\
\hline 68 & 4 & 1 & 3 & 3 & 1 & 2 & E & 12171.9356 & -0.6 \\
\hline 69 & 5 & 1 & 4 & 4 & 1 & 3 & A & 15136.7682 & 1.1 \\
\hline 70 & 5 & 1 & 4 & 4 & 1 & 3 & E & 15135.6994 & -1.0 \\
\hline 71 & 6 & 1 & 5 & 5 & 1 & 4 & A & 18037.5873 & 0.8 \\
\hline 72 & 6 & 1 & 5 & 5 & 1 & 4 & E & 18036.3994 & -1.2 \\
\hline 73 & 7 & 1 & 6 & 6 & 1 & 5 & A & 20854.7687 & 1.0 \\
\hline 74 & 7 & 1 & 6 & 6 & 1 & 5 & E & 20853.5374 & -1.4 \\
\hline 75 & 8 & 1 & 7 & 7 & 1 & 6 & A & 23569.3884 & 1.4 \\
\hline 76 & 8 & 1 & 7 & 7 & 1 & 6 & E & 23568.1984 & -0.7 \\
\hline 77 & 4 & 1 & 3 & 4 & 1 & 4 & A & 3993.9050 & 4.9 \\
\hline 78 & 4 & 1 & 3 & 4 & 1 & 4 & $\mathrm{E}$ & 3992.5858 & -0.4 \\
\hline 79 & 5 & 1 & 4 & 5 & 1 & 5 & A & 5957.6426 & 7.2 \\
\hline 80 & 5 & 1 & 4 & 5 & 1 & 5 & E & 5955.5124 & -1.1 \\
\hline 81 & 6 & 1 & 5 & 6 & 1 & 6 & A & 8257.6093 & -8.2 \\
\hline
\end{tabular}




\begin{tabular}{|c|c|c|c|c|c|c|c|c|c|}
\hline 82 & 6 & 1 & 5 & 6 & 1 & 6 & $\mathrm{E}$ & 8254.5893 & -0.9 \\
\hline 83 & 7 & 1 & 6 & 7 & 1 & 7 & A & 10835.5692 & -6.5 \\
\hline 84 & 7 & 1 & 6 & 7 & 1 & 7 & $E$ & 10831.6025 & -2.9 \\
\hline 85 & 8 & 1 & 7 & 8 & 1 & 8 & A & 13610.8567 & -3.6 \\
\hline 86 & 8 & 1 & 7 & 8 & 1 & 8 & $\mathrm{E}$ & 13605.9810 & -3.4 \\
\hline 87 & 2 & 1 & 2 & 1 & 1 & 1 & A & 5323.1136 & -0.7 \\
\hline 88 & 2 & 1 & 2 & 1 & 1 & 1 & $E$ & 5323.3907 & 0.6 \\
\hline 89 & 4 & 1 & 4 & 3 & 1 & 3 & A & 10581.4279 & -0.1 \\
\hline 90 & 4 & 1 & 4 & 3 & 1 & 3 & $E$ & 10581.2179 & -0.2 \\
\hline 91 & 3 & 1 & 3 & 2 & 1 & 2 & A & 7963.6040 & -4.4 \\
\hline 92 & 3 & 1 & 3 & 2 & 1 & 2 & $E$ & 7963.5060 & -0.2 \\
\hline 93 & 5 & 1 & 5 & 4 & 1 & 4 & A & 13173.0321 & 0.4 \\
\hline 94 & 5 & 1 & 5 & 4 & 1 & 4 & $E$ & 13172.7729 & -0.2 \\
\hline 95 & 6 & 1 & 6 & 5 & 1 & 5 & A & 15737.6044 & 0.0 \\
\hline 96 & 6 & 1 & 6 & 5 & 1 & 5 & $E$ & 15737.3238 & -0.2 \\
\hline 97 & 7 & 1 & 7 & 6 & 1 & 6 & A & 18276.8089 & -0.7 \\
\hline 98 & 7 & 1 & 7 & 6 & 1 & 6 & $E$ & 18276.5231 & -0.4 \\
\hline 99 & 8 & 1 & 8 & 7 & 1 & 7 & A & 20794.1016 & -0.8 \\
\hline 100 & 8 & 1 & 8 & 7 & 1 & 7 & $E$ & 20793.8198 & -0.4 \\
\hline 101 & 9 & 1 & 9 & 8 & 1 & 8 & A & 23293.8219 & -1.1 \\
\hline 102 & 9 & 1 & 9 & 8 & 1 & 8 & $\mathrm{E}$ & 23293.5483 & -1.5 \\
\hline 103 & 5 & 1 & 4 & 4 & 2 & 3 & A & 6804.1628 & 6.6 \\
\hline 104 & 5 & 1 & 4 & 4 & 2 & 3 & E & 6805.5256 & -0.7 \\
\hline 105 & 6 & 1 & 5 & 5 & 2 & 4 & A & 10610.1206 & 1.2 \\
\hline 106 & 6 & 1 & 5 & 5 & 2 & 4 & E & 10609.8903 & -2.1 \\
\hline 107 & 7 & 1 & 6 & 6 & 2 & 5 & A & 14450.5468 & 5.6 \\
\hline 108 & 7 & 1 & 6 & 6 & 2 & 5 & $E$ & 14449.4549 & -3.6 \\
\hline 109 & 8 & 1 & 7 & 7 & 2 & 6 & A & 18256.0637 & 2.7 \\
\hline 110 & 8 & 1 & 7 & 7 & 2 & 6 & $E$ & 18254.4671 & -4.4 \\
\hline 111 & 9 & 1 & 8 & 8 & 2 & 7 & A & 21951.4851 & 2.5 \\
\hline 112 & 9 & 1 & 8 & 8 & 2 & 7 & $\mathrm{E}$ & 21949.6352 & -4.5 \\
\hline 113 & 3 & 2 & 1 & 2 & 0 & 2 & A & 22480.4817 & 1.7 \\
\hline 114 & 3 & 2 & 1 & 2 & 0 & 2 & $\mathrm{E}$ & 22484.1084 & 2.9 \\
\hline 115 & 3 & 2 & 2 & 3 & 0 & 3 & A & 13859.5954 & 1.2 \\
\hline 116 & 3 & 2 & 2 & 3 & 0 & 3 & $\mathrm{E}$ & 13852.4653 & 2.4 \\
\hline 117 & 4 & 2 & 3 & 4 & 0 & 4 & A & 14168.2270 & 1.9 \\
\hline 118 & 4 & 2 & 3 & 4 & 0 & 4 & $E$ & 14164.6203 & 3.4 \\
\hline 119 & 5 & 2 & 4 & 5 & 0 & 5 & A & 14723.5255 & 1.6 \\
\hline 120 & 5 & 2 & 4 & 5 & 0 & 5 & $E$ & 14720.6879 & 3.4 \\
\hline 121 & 6 & 2 & 5 & 6 & 0 & 6 & A & 15578.1392 & -0.8 \\
\hline 122 & 6 & 2 & 5 & 6 & 0 & 6 & $\mathrm{E}$ & 15575.2332 & 3.1 \\
\hline 123 & 7 & 2 & 6 & 7 & 0 & 7 & A & 16745.8364 & -1.4 \\
\hline 124 & 7 & 2 & 6 & 7 & 0 & 7 & $E$ & 16742.5081 & 2.6 \\
\hline
\end{tabular}




\begin{tabular}{|c|c|c|c|c|c|c|c|c|c|}
\hline 125 & 8 & 2 & 7 & 8 & 0 & 8 & A & 18205.4880 & 0.3 \\
\hline 126 & 8 & 2 & 7 & 8 & 0 & 8 & $E$ & 18201.5650 & 3.9 \\
\hline 127 & 2 & 2 & 0 & 1 & 1 & 1 & A & 16223.6784 & 2.5 \\
\hline 128 & 2 & 2 & 0 & 1 & 1 & 1 & $E$ & 16241.6788 & -6.7 \\
\hline 129 & 3 & 2 & 1 & 2 & 1 & 2 & A & 19625.0287 & 4.7 \\
\hline 130 & 3 & 2 & 1 & 2 & 1 & 2 & E & 19628.9788 & 1.2 \\
\hline 131 & 4 & 2 & 2 & 3 & 1 & 3 & A & 23417.6407 & -7.7 \\
\hline 132 & 4 & 2 & 2 & 3 & 1 & 3 & E & 23417.2515 & 1.5 \\
\hline 133 & 2 & 2 & 0 & 1 & 1 & 0 & E & 15839.7948 & -6.6 \\
\hline 134 & 2 & 2 & 1 & 1 & 1 & 0 & A & 15787.8239 & -2.0 \\
\hline 135 & 2 & 2 & 1 & 1 & 1 & 0 & $E$ & 15766.9489 & 6.9 \\
\hline 136 & 3 & 2 & 2 & 2 & 1 & 1 & A & 18249.0059 & -2.3 \\
\hline 137 & 3 & 2 & 2 & 2 & 1 & 1 & $E$ & 18241.7930 & -1.4 \\
\hline 138 & 4 & 2 & 3 & 3 & 1 & 2 & A & 20505.4449 & -3.5 \\
\hline 139 & 5 & 2 & 4 & 4 & 1 & 3 & A & 22564.2291 & -5.1 \\
\hline 140 & 5 & 2 & 4 & 4 & 1 & 3 & E & 22562.2085 & -0.2 \\
\hline 141 & 6 & 2 & 5 & 5 & 1 & 4 & A & 24441.8071 & -5.9 \\
\hline 142 & 6 & 2 & 5 & 5 & 1 & 4 & E & 24440.4821 & 1.2 \\
\hline 143 & 2 & 2 & 0 & 2 & 1 & 1 & A & 9698.3456 & -3.3 \\
\hline 144 & 2 & 2 & 0 & 2 & 1 & 1 & E & 9716.0597 & 1.3 \\
\hline 145 & 2 & 2 & 1 & 2 & 1 & 1 & E & 9643.1979 & -1.1 \\
\hline 146 & 3 & 2 & 2 & 3 & 1 & 2 & E & 9078.6561 & -1.0 \\
\hline 147 & 2 & 2 & 0 & 2 & 1 & 2 & E & 10918.2971 & 1.7 \\
\hline 148 & 3 & 2 & 1 & 3 & 1 & 3 & E & 11665.4724 & 1.1 \\
\hline 149 & 2 & 2 & 1 & 2 & 1 & 2 & A & 10865.4508 & 1.4 \\
\hline 150 & 2 & 2 & 1 & 2 & 1 & 2 & $E$ & 10845.4337 & -2.3 \\
\hline 151 & 3 & 2 & 2 & 3 & 1 & 3 & A & 11487.6124 & 0.1 \\
\hline 152 & 3 & 2 & 2 & 3 & 1 & 3 & $E$ & 11480.5246 & -0.6 \\
\hline 153 & 4 & 2 & 3 & 4 & 1 & 4 & A & 12326.5171 & 6.2 \\
\hline 154 & 4 & 2 & 3 & 4 & 1 & 4 & $E$ & 12322.7614 & 1.0 \\
\hline 155 & 5 & 2 & 4 & 5 & 1 & 5 & A & 13385.1096 & 7.1 \\
\hline 156 & 5 & 2 & 4 & 5 & 1 & 5 & E & 13382.0224 & 0.6 \\
\hline 157 & 6 & 2 & 5 & 6 & 1 & 6 & A & 14661.8417 & -2.4 \\
\hline 158 & 6 & 2 & 5 & 6 & 1 & 6 & E & 14658.6716 & 1.1 \\
\hline 159 & 7 & 2 & 6 & 7 & 1 & 7 & A & 16148.8996 & -2.1 \\
\hline 160 & 7 & 2 & 6 & 7 & 1 & 7 & E & 16145.3347 & 1.6 \\
\hline 161 & 8 & 2 & 7 & 8 & 1 & 8 & A & 17831.4633 & -0.5 \\
\hline 162 & 8 & 2 & 7 & 8 & 1 & 8 & E & 17827.3512 & 1.7 \\
\hline 163 & 9 & 2 & 8 & 9 & 1 & 9 & A & 19688.3974 & 1.7 \\
\hline 164 & 9 & 2 & 8 & 9 & 1 & 9 & $E$ & 19683.6550 & 2.2 \\
\hline 165 & 3 & 2 & 1 & 3 & 1 & 2 & A & 9258.9218 & -3.3 \\
\hline 166 & 3 & 2 & 1 & 3 & 1 & 2 & $E$ & 9263.6023 & -1.0 \\
\hline 167 & 4 & 2 & 2 & 4 & 1 & 3 & A & 8842.3211 & 0.7 \\
\hline
\end{tabular}




\begin{tabular}{|c|c|c|c|c|c|c|c|c|c|}
\hline 168 & 4 & 2 & 2 & 4 & 1 & 3 & $\mathrm{E}$ & 8843.4457 & 0.0 \\
\hline 169 & 5 & 2 & 3 & 5 & 1 & 4 & A & 8569.7496 & 0.7 \\
\hline 170 & 5 & 2 & 3 & 5 & 1 & 4 & $\mathrm{E}$ & 8569.8151 & 2.2 \\
\hline 171 & 6 & 2 & 4 & 6 & 1 & 5 & A & 8555.1361 & 0.6 \\
\hline 172 & 6 & 2 & 4 & 6 & 1 & 5 & $\mathrm{E}$ & 8554.6654 & 4.8 \\
\hline 173 & 7 & 2 & 5 & 7 & 1 & 6 & A & 8891.0817 & 1.5 \\
\hline 174 & 7 & 2 & 5 & 7 & 1 & 6 & $\mathrm{E}$ & 8890.0798 & 9.3 \\
\hline 175 & 8 & 2 & 6 & 8 & 1 & 7 & A & 9648.4948 & -7.7 \\
\hline 176 & 2 & 2 & 1 & 1 & 1 & 1 & $\mathrm{E}$ & 16168.8322 & 6.1 \\
\hline 177 & 3 & 2 & 1 & 2 & 1 & 1 & $\mathrm{E}$ & 18426.7399 & -0.6 \\
\hline 178 & 3 & 2 & 2 & 2 & 1 & 2 & $\mathrm{E}$ & 19444.0313 & -0.2 \\
\hline 179 & 3 & 2 & 2 & 2 & 2 & 1 & A & 8585.7719 & 0.6 \\
\hline 180 & 3 & 2 & 2 & 2 & 2 & 1 & $\mathrm{E}$ & 8598.5979 & 2.5 \\
\hline 181 & 4 & 2 & 3 & 3 & 2 & 2 & A & 11420.3266 & 0.1 \\
\hline 182 & 4 & 2 & 3 & 3 & 2 & 2 & $\mathrm{E}$ & 11423.4539 & 0.6 \\
\hline 183 & 5 & 2 & 4 & 4 & 2 & 3 & A & 14231.6233 & 0.0 \\
\hline 184 & 5 & 2 & 4 & 4 & 2 & 3 & $\mathrm{E}$ & 14232.0353 & 0.8 \\
\hline 185 & 6 & 2 & 5 & 5 & 2 & 4 & A & 17014.3467 & 0.8 \\
\hline 186 & 6 & 2 & 5 & 5 & 2 & 4 & $\mathrm{E}$ & 17013.9727 & 0.0 \\
\hline 187 & 7 & 2 & 6 & 6 & 2 & 5 & A & 19763.8678 & 0.6 \\
\hline 188 & 7 & 2 & 6 & 6 & 2 & 5 & $\mathrm{E}$ & 19763.1865 & 0.4 \\
\hline 189 & 8 & 2 & 7 & 7 & 2 & 6 & A & 22476.6650 & 0.5 \\
\hline 190 & 8 & 2 & 7 & 7 & 2 & 6 & $\mathrm{E}$ & 22475.8366 & 0.1 \\
\hline 191 & 9 & 2 & 8 & 8 & 2 & 7 & A & 25150.7552 & 0.3 \\
\hline 192 & 9 & 2 & 8 & 8 & 2 & 7 & $\mathrm{E}$ & 25149.8523 & -0.8 \\
\hline 193 & 4 & 2 & 2 & 3 & 2 & 1 & A & 11756.2333 & 0.5 \\
\hline 194 & 4 & 2 & 2 & 3 & 2 & 1 & $E$ & 11751.7796 & 1.0 \\
\hline 195 & 5 & 2 & 3 & 4 & 2 & 2 & A & 14864.1967 & 1.1 \\
\hline 196 & 5 & 2 & 3 & 4 & 2 & 2 & $E$ & 14862.0689 & 1.2 \\
\hline 197 & 6 & 2 & 4 & 5 & 2 & 3 & A & 18022.9742 & 1.0 \\
\hline 198 & 6 & 2 & 4 & 5 & 2 & 3 & $\mathrm{E}$ & 18021.2496 & 1.3 \\
\hline 199 & 7 & 2 & 5 & 6 & 2 & 4 & A & 21190.7137 & 1.3 \\
\hline 200 & 7 & 2 & 5 & 6 & 2 & 4 & $\mathrm{E}$ & 21188.9497 & 1.0 \\
\hline 201 & 8 & 2 & 6 & 7 & 2 & 5 & A & 24326.8113 & 1.9 \\
\hline 202 & 8 & 2 & 6 & 7 & 2 & 5 & $E$ & 24324.9002 & 0.0 \\
\hline 203 & 4 & 2 & 2 & 3 & 2 & 2 & $\mathrm{E}$ & 11936.7265 & 1.7 \\
\hline 204 & 4 & 2 & 3 & 3 & 2 & 1 & $\mathrm{E}$ & 11238.5072 & 0.1 \\
\hline 205 & 8 & 2 & 6 & 7 & 3 & 5 & A & 9749.7011 & -1.6 \\
\hline 206 & 8 & 2 & 6 & 7 & 3 & 5 & $\mathrm{E}$ & 9754.2146 & 3.2 \\
\hline 207 & 9 & 2 & 7 & 8 & 3 & 6 & A & 14039.4541 & 0.3 \\
\hline 208 & 3 & 3 & 0 & 3 & 2 & 2 & $\mathrm{E}$ & 17180.8486 & 0.3 \\
\hline 209 & 4 & 3 & 1 & 4 & 2 & 3 & $\mathrm{E}$ & 17275.3506 & 0.7 \\
\hline 210 & 5 & 3 & 2 & 5 & 2 & 4 & $\mathrm{E}$ & 17480.0572 & -2.3 \\
\hline
\end{tabular}




\begin{tabular}{|c|c|c|c|c|c|c|c|c|c|}
\hline 211 & 3 & 3 & 1 & 3 & 2 & 2 & A & 17128.2716 & 0.5 \\
\hline 212 & 3 & 3 & 1 & 3 & 2 & 2 & $\mathrm{E}$ & 17085.0716 & -3.6 \\
\hline 213 & 4 & 3 & 2 & 4 & 2 & 3 & A & 17220.4189 & 1.0 \\
\hline 214 & 4 & 3 & 2 & 4 & 2 & 3 & $\mathrm{E}$ & 17178.8854 & -3.5 \\
\hline 215 & 5 & 3 & 3 & 5 & 2 & 4 & A & 17400.0900 & 1.0 \\
\hline 216 & 5 & 3 & 3 & 5 & 2 & 4 & $\mathrm{E}$ & 17371.0154 & -0.5 \\
\hline 217 & 6 & 3 & 4 & 6 & 2 & 5 & A & 17700.8577 & 1.1 \\
\hline 218 & 6 & 3 & 4 & 6 & 2 & 5 & $\mathrm{E}$ & 17685.9934 & 1.0 \\
\hline 219 & 7 & 3 & 5 & 7 & 2 & 6 & A & 18154.8612 & 0.3 \\
\hline 220 & 7 & 3 & 5 & 7 & 2 & 6 & $\mathrm{E}$ & 18147.0340 & 2.3 \\
\hline 221 & 8 & 3 & 6 & 8 & 2 & 7 & A & 18789.9625 & 1.5 \\
\hline 222 & 8 & 3 & 6 & 8 & 2 & 7 & $\mathrm{E}$ & 18784.7018 & 1.5 \\
\hline 223 & 3 & 3 & 1 & 3 & 2 & 1 & $\mathrm{E}$ & 16900.1237 & -5.4 \\
\hline 224 & 4 & 3 & 2 & 4 & 2 & 2 & $\mathrm{E}$ & 16665.6147 & -2.7 \\
\hline 225 & 5 & 3 & 3 & 5 & 2 & 3 & $\mathrm{E}$ & 16227.7104 & -0.9 \\
\hline 226 & 6 & 3 & 4 & 6 & 2 & 4 & $\mathrm{E}$ & 15535.4098 & -2.3 \\
\hline 227 & 3 & 3 & 0 & 3 & 2 & 1 & A & 16956.4006 & 1.1 \\
\hline 228 & 3 & 3 & 0 & 3 & 2 & 1 & $\mathrm{E}$ & 16995.9017 & -0.5 \\
\hline 229 & 4 & 3 & 1 & 4 & 2 & 2 & A & 16724.1731 & 1.2 \\
\hline 230 & 4 & 3 & 1 & 4 & 2 & 2 & $\mathrm{E}$ & 16762.0776 & -0.8 \\
\hline 231 & 5 & 3 & 2 & 5 & 2 & 3 & A & 16311.2019 & 0.8 \\
\hline 232 & 5 & 3 & 2 & 5 & 2 & 3 & $\mathrm{E}$ & 16336.7516 & -3.3 \\
\hline 233 & 6 & 3 & 3 & 6 & 2 & 4 & A & 15707.7150 & -0.3 \\
\hline 234 & 6 & 3 & 3 & 6 & 2 & 4 & $\mathrm{E}$ & 15719.1545 & -4.3 \\
\hline 235 & 7 & 3 & 4 & 7 & 2 & 5 & A & 14961.8244 & -2.5 \\
\hline 236 & 7 & 3 & 4 & 7 & 2 & 5 & $E$ & 14966.2562 & -4.5 \\
\hline 237 & 8 & 3 & 5 & 8 & 2 & 6 & A & 14176.5275 & -0.3 \\
\hline 238 & 8 & 3 & 5 & 8 & 2 & 6 & $E$ & 14178.2553 & -3.3 \\
\hline 239 & 9 & 3 & 6 & 9 & 2 & 7 & A & 13488.9616 & -0.9 \\
\hline 240 & 9 & 3 & 6 & 9 & 2 & 7 & $E$ & 13489.5316 & 1.0 \\
\hline 241 & 4 & 3 & 2 & 3 & 3 & 1 & A & 11512.4739 & 0.5 \\
\hline 242 & 4 & 3 & 2 & 3 & 3 & 1 & $\mathrm{E}$ & 11517.2698 & 2.8 \\
\hline 243 & 5 & 3 & 3 & 4 & 3 & 2 & A & 14411.2945 & 0.1 \\
\hline 244 & 5 & 3 & 3 & 4 & 3 & 2 & $\mathrm{E}$ & 14424.1639 & 2.4 \\
\hline 245 & 6 & 3 & 4 & 5 & 3 & 3 & A & 17315.1142 & 0.6 \\
\hline 246 & 6 & 3 & 4 & 5 & 3 & 3 & $E$ & 17328.9507 & 1.5 \\
\hline 247 & 7 & 3 & 5 & 6 & 3 & 4 & A & 20217.8722 & 0.7 \\
\hline 248 & 7 & 3 & 5 & 6 & 3 & 4 & $\mathrm{E}$ & 20224.2261 & 0.7 \\
\hline 249 & 8 & 3 & 6 & 7 & 3 & 5 & A & 23111.7654 & 0.7 \\
\hline 250 & 8 & 3 & 6 & 7 & 3 & 5 & $E$ & 23113.5058 & 0.7 \\
\hline 251 & 4 & 3 & 1 & 3 & 3 & 0 & A & 11524.0075 & 2.3 \\
\hline 252 & 4 & 3 & 1 & 3 & 3 & 0 & $\mathrm{E}$ & 11517.9552 & 0.3 \\
\hline 253 & 5 & 3 & 2 & 4 & 3 & 1 & A & 14451.2248 & 0.0 \\
\hline
\end{tabular}




\begin{tabular}{llllllllrr}
\hline $\mathbf{2 5 4}$ & 5 & 3 & 2 & 4 & 3 & 1 & $\mathrm{E}$ & 14436.7425 & -1.6 \\
$\mathbf{2 5 5}$ & 6 & 3 & 3 & 5 & 3 & 2 & $\mathrm{~A}$ & 17419.4877 & 0.3 \\
$\mathbf{2 5 6}$ & 6 & 3 & 3 & 5 & 3 & 2 & $\mathrm{E}$ & 17403.6528 & 0.5 \\
$\mathbf{2 5 7}$ & 7 & 3 & 4 & 6 & 3 & 3 & $\mathrm{~A}$ & 20444.8246 & 0.6 \\
$\mathbf{2 5 8}$ & 7 & 3 & 4 & 6 & 3 & 3 & $\mathrm{E}$ & 20436.0518 & 1.2 \\
$\mathbf{2 5 9}$ & 8 & 3 & 5 & 7 & 3 & 4 & $\mathrm{~A}$ & 23541.5121 & 1.8 \\
$\mathbf{2 6 0}$ & 8 & 3 & 5 & 7 & 3 & 4 & $\mathrm{E}$ & 23536.9009 & 2.7 \\
$\mathbf{2 6 1}$ & 4 & 4 & 0 & 4 & 3 & 1 & $\mathrm{~A}$ & 23878.1739 & 0.7 \\
$\mathbf{2 6 2}$ & 4 & 4 & 0 & 4 & 3 & 1 & $\mathrm{E}$ & 23897.7996 & -2.2 \\
$\mathbf{2 6 3}$ & 6 & 4 & 2 & 6 & 3 & 3 & $\mathrm{~A}$ & 23688.7381 & -0.2 \\
$\mathbf{2 6 4}$ & 4 & 4 & 1 & 5 & 3 & 2 & $\mathrm{~A}$ & 9426.8607 & 0.3 \\
$\mathbf{2 6 5}$ & 4 & 4 & 0 & 5 & 3 & 3 & $\mathrm{~A}$ & 9480.3486 & 6.2 \\
$\mathbf{2 6 6}$ & 4 & 4 & 1 & 4 & 3 & 2 & $\mathrm{~A}$ & 23891.5480 & -0.7 \\
$\mathbf{2 6 7}$ & 4 & 4 & 1 & 4 & 3 & 2 & $\mathrm{E}$ & 23866.7365 & -1.6 \\
$\mathbf{2 6 8}$ & 5 & 4 & 2 & 5 & 3 & 3 & $\mathrm{~A}$ & 23867.8100 & 0.4 \\
$\mathbf{2 6 9}$ & 6 & 4 & 3 & 6 & 3 & 4 & $\mathrm{~A}$ & 23842.5734 & 0.4 \\
$\mathbf{2 7 0}$ & 6 & 4 & 3 & 6 & 3 & 4 & $\mathrm{E}$ & 23791.5169 & 0.7 \\
$\mathbf{2 7 1}$ & 8 & 4 & 5 & 8 & 3 & 6 & $\mathrm{~A}$ & 23847.7796 & 4.2 \\
$\mathbf{2 7 2}$ & 5 & 4 & 2 & 4 & 4 & 1 & $\mathrm{~A}$ & 14387.5557 & 0.4 \\
$\mathbf{2 7 3}$ & 5 & 4 & 2 & 4 & 4 & 1 & $\mathrm{E}$ & 14387.2496 & 4.1 \\
$\mathbf{2 7 4}$ & 6 & 4 & 3 & 5 & 4 & 2 & $\mathrm{~A}$ & 17289.8776 & 0.6 \\
$\mathbf{2 7 5}$ & 6 & 4 & 3 & 5 & 4 & 2 & $\mathrm{E}$ & 17290.6471 & 3.7 \\
$\mathbf{2 7 6}$ & 7 & 4 & 4 & 6 & 4 & 3 & $\mathrm{~A}$ & 20204.2344 & 0.3 \\
$\mathbf{2 7 7}$ & 7 & 4 & 4 & 6 & 4 & 3 & $\mathrm{E}$ & 20208.1698 & 3.1 \\
$\mathbf{2 7 8}$ & 8 & 4 & 5 & 7 & 4 & 4 & $\mathrm{~A}$ & 23130.6044 & -0.1 \\
$\mathbf{2 7 9}$ & 8 & 4 & 5 & 7 & 4 & 4 & $\mathrm{E}$ & 23140.6139 & 5.1 \\
$\mathbf{2 8 0}$ & 5 & 4 & 1 & 4 & 4 & 0 & $\mathrm{~A}$ & 14388.2579 & 0.2 \\
$\mathbf{2 8 1}$ & 5 & 4 & 1 & 4 & 4 & 0 & $\mathrm{E}$ & 14387.0090 & -1.9 \\
$\mathbf{2 8 2}$ & 6 & 4 & 2 & 5 & 4 & 1 & $\mathrm{~A}$ & 17293.0195 & 0.1 \\
$\mathbf{2 8 3}$ & 6 & 4 & 2 & 5 & 4 & 1 & $\mathrm{E}$ & 17290.3497 & -2.1 \\
$\mathbf{2 8 4}$ & 7 & 4 & 3 & 6 & 4 & 2 & $\mathrm{~A}$ & 20214.6075 & 0.2 \\
$\mathbf{2 8 5}$ & 7 & 4 & 3 & 6 & 4 & 2 & $\mathrm{E}$ & 20208.4081 & -2.1 \\
$\mathbf{2 8 6}$ & 8 & 4 & 4 & 7 & 4 & 3 & $\mathrm{~A}$ & 23158.7010 & 0.1 \\
$\mathbf{2 8 7}$ & 8 & 4 & 4 & 7 & 4 & 3 & $\mathrm{E}$ & 23146.0435 & -2.8 \\
$\mathbf{2 8 8}$ & 6 & 5 & 1 & 5 & 5 & 0 & $\mathrm{~A}$ & 17259.5709 & -8.5 \\
$\mathbf{2 8 9}$ & 6 & 5 & 1 & 5 & 5 & 0 & $\mathrm{E}$ & 17258.4939 & -3.1 \\
$\mathbf{2 9 0}$ & 6 & 5 & 2 & 5 & 5 & 1 & $\mathrm{~A}$ & 17259.5507 & 7.5 \\
$\mathbf{2 9 1}$ & 6 & 5 & 2 & 5 & 5 & 1 & $\mathrm{E}$ & 17258.7777 & 6.0 \\
$\mathbf{2 9 2}$ & 8 & 6 & 2 & 7 & 6 & 1 & $\mathrm{~A}$ & 23028.1255 & -5.5 \\
$\mathbf{2 9 3}$ & 8 & 6 & 2 & 7 & 6 & 1 & $\mathrm{E}$ & 23026.6750 & -5.6 \\
\hline & & & & & & & & &
\end{tabular}


Table S6: Observed frequencies $v_{o b s}$ of 213 rotational transitions of $s y n-2 A F$ with $v_{o b s}-v_{c a l c}$ values obtained from a fit with the program XIAM.

\begin{tabular}{|c|c|c|c|c|c|c|c|c|c|}
\hline \multirow[t]{2}{*}{ No. } & $\boldsymbol{J}$ & $K_{a}$ & $K_{c}$ & $J$ & $K_{a}$ & $K_{c}$ & \multirow{2}{*}{ Species } & \multirow{2}{*}{$\begin{array}{c}\text { Vobs } \\
{[\mathrm{MHz}]}\end{array}$} & \multirow{2}{*}{$\begin{array}{c}\mathbf{v}_{\text {obs }}-\mathbf{v}_{\text {calc }} \\
{[\mathrm{kHz}]}\end{array}$} \\
\hline & \multicolumn{3}{|c|}{ upper level } & \multicolumn{3}{|c|}{ lower level } & & & \\
\hline 1 & 1 & 0 & 1 & 0 & 0 & 0 & $A$ & 2840.0347 & 0.7 \\
\hline 2 & 1 & 0 & 1 & 0 & 0 & 0 & $E$ & 2839.4376 & 0.4 \\
\hline 3 & 2 & 0 & 2 & 1 & 0 & 1 & $A$ & 5647.2818 & 1.2 \\
\hline 4 & 2 & 0 & 2 & 1 & 0 & 1 & $E$ & 5646.2312 & 0.1 \\
\hline 5 & 3 & 0 & 3 & 2 & 0 & 2 & $A$ & 8390.4604 & 0.2 \\
\hline 6 & 3 & 0 & 3 & 2 & 0 & 2 & $E$ & 8389.1862 & -0.1 \\
\hline 7 & 4 & 0 & 4 & 3 & 0 & 3 & $A$ & 11045.2937 & 0.5 \\
\hline 8 & 4 & 0 & 4 & 3 & 0 & 3 & $E$ & 11044.0149 & 0.0 \\
\hline 9 & 5 & 0 & 5 & 4 & 0 & 4 & $A$ & 13604.2470 & -0.8 \\
\hline 10 & 5 & 0 & 5 & 4 & 0 & 4 & $E$ & 13603.0981 & -0.7 \\
\hline 11 & 6 & 0 & 6 & 5 & 0 & 5 & $A$ & 16082.7797 & 0.0 \\
\hline 12 & 6 & 0 & 6 & 5 & 0 & 5 & $E$ & 16081.7879 & 0.2 \\
\hline 13 & 7 & 0 & 7 & 6 & 0 & 6 & $A$ & 18512.1622 & -0.7 \\
\hline 14 & 7 & 0 & 7 & 6 & 0 & 6 & $E$ & 18511.2892 & -0.6 \\
\hline 15 & 8 & 0 & 8 & 7 & 0 & 7 & $A$ & 20922.9796 & -0.4 \\
\hline 16 & 8 & 0 & 8 & 7 & 0 & 7 & $E$ & 20922.1755 & 0.4 \\
\hline 17 & 9 & 0 & 9 & 8 & 0 & 8 & $A$ & 23334.2474 & 0.2 \\
\hline 18 & 9 & 0 & 9 & 8 & 0 & 8 & $E$ & 23333.4743 & -0.6 \\
\hline 19 & 3 & 0 & 3 & 2 & 1 & 2 & $A$ & 5459.9605 & -2.4 \\
\hline 20 & 3 & 0 & 3 & 2 & 1 & 2 & $E$ & 5462.8335 & 0.1 \\
\hline 21 & 4 & 0 & 4 & 3 & 1 & 3 & $A$ & 8590.9116 & -0.7 \\
\hline 22 & 4 & 0 & 4 & 3 & 1 & 3 & $E$ & 8591.5079 & 1.1 \\
\hline 23 & 5 & 0 & 5 & 4 & 1 & 4 & $A$ & 11677.1447 & -0.2 \\
\hline 24 & 5 & 0 & 5 & 4 & 1 & 4 & $E$ & 11676.6972 & 0.2 \\
\hline 25 & 6 & 0 & 6 & 5 & 1 & 5 & $A$ & 14663.0384 & 0.8 \\
\hline 26 & 6 & 0 & 6 & 5 & 1 & 5 & $E$ & 14662.1161 & -1.3 \\
\hline 27 & 7 & 0 & 7 & 6 & 1 & 6 & $A$ & 17525.2443 & 0.8 \\
\hline 28 & 7 & 0 & 7 & 6 & 1 & 6 & $E$ & 17524.1563 & -0.2 \\
\hline 29 & 8 & 0 & 8 & 7 & 1 & 7 & $A$ & 20269.6905 & 0.7 \\
\hline 30 & 8 & 0 & 8 & 7 & 1 & 7 & $E$ & 20268.5897 & 0.7 \\
\hline 31 & 9 & 0 & 9 & 8 & 1 & 8 & $A$ & 22918.2807 & 1.5 \\
\hline 32 & 9 & 0 & 9 & 8 & 1 & 8 & $E$ & 22917.2305 & 1.4 \\
\hline 33 & 1 & 1 & 0 & 1 & 0 & 1 & $A$ & 3679.1205 & 1.3 \\
\hline 34 & 2 & 1 & 1 & 2 & 0 & 2 & $A$ & 4102.6105 & 2.9 \\
\hline 35 & 3 & 1 & 2 & 3 & 0 & 3 & $E$ & 4796.5656 & 0.6 \\
\hline 36 & 4 & 1 & 3 & 4 & 0 & 4 & $A$ & 5822.0824 & -4.3 \\
\hline 37 & 4 & 1 & 3 & 4 & 0 & 4 & $E$ & 5820.0571 & 2.3 \\
\hline 38 & 5 & 1 & 4 & 5 & 0 & 5 & A & 7232.4777 & -2.7 \\
\hline
\end{tabular}




\begin{tabular}{|c|c|c|c|c|c|c|c|c|c|}
\hline 39 & 5 & 1 & 4 & 5 & 0 & 5 & $E$ & 7228.2040 & 0.2 \\
\hline 40 & 6 & 1 & 5 & 6 & 0 & 6 & A & 9050.2240 & -4.4 \\
\hline 41 & 6 & 1 & 5 & 6 & 0 & 6 & $\mathrm{E}$ & 9043.1578 & 0.1 \\
\hline 42 & 8 & 1 & 7 & 8 & 0 & 8 & A & 13746.3717 & -1.5 \\
\hline 43 & 8 & 1 & 7 & 8 & 0 & 8 & $E$ & 13733.1745 & -1.6 \\
\hline 44 & 1 & 1 & 0 & 0 & 0 & 0 & $E$ & 6526.3853 & 1.3 \\
\hline 45 & 1 & 1 & 1 & 0 & 0 & 0 & A & 6128.4489 & -0.5 \\
\hline 46 & 2 & 1 & 2 & 1 & 0 & 1 & A & 8577.7817 & 3.8 \\
\hline 47 & 2 & 1 & 2 & 1 & 0 & 1 & $\mathrm{E}$ & 8572.5847 & 0.6 \\
\hline 48 & 3 & 1 & 3 & 2 & 0 & 2 & A & 10844.8410 & -0.1 \\
\hline 49 & 3 & 1 & 3 & 2 & 0 & 2 & $\mathrm{E}$ & 10841.6965 & 2.1 \\
\hline 50 & 4 & 1 & 4 & 3 & 0 & 3 & A & 12972.3982 & 2.0 \\
\hline 51 & 4 & 1 & 4 & 3 & 0 & 3 & $E$ & 12970.4168 & 0.1 \\
\hline 52 & 5 & 1 & 5 & 4 & 0 & 4 & A & 15023.9913 & 1.4 \\
\hline 53 & 5 & 1 & 5 & 4 & 0 & 4 & $E$ & 15022.7680 & -1.1 \\
\hline 54 & 6 & 1 & 6 & 5 & 0 & 5 & A & 17069.6989 & -0.2 \\
\hline 55 & 6 & 1 & 6 & 5 & 0 & 5 & $\mathrm{E}$ & 17068.9208 & -0.3 \\
\hline 56 & 7 & 1 & 7 & 6 & 0 & 6 & A & 19165.4508 & -2.2 \\
\hline 57 & 7 & 1 & 7 & 6 & 0 & 6 & $\mathrm{E}$ & 19164.8755 & -0.5 \\
\hline 58 & 8 & 1 & 8 & 7 & 0 & 7 & A & 21338.9472 & -0.7 \\
\hline 59 & 8 & 1 & 8 & 7 & 0 & 7 & $E$ & 21338.4201 & -0.8 \\
\hline 60 & 9 & 1 & 9 & 8 & 0 & 8 & A & 23591.2716 & -0.5 \\
\hline 61 & 9 & 1 & 9 & 8 & 0 & 8 & $E$ & 23590.7230 & -0.1 \\
\hline 62 & 5 & 1 & 4 & 5 & 1 & 5 & A & 5812.7346 & -3.7 \\
\hline 63 & 5 & 1 & 4 & 5 & 1 & 5 & $\mathrm{E}$ & 5808.5337 & 0.2 \\
\hline 64 & 2 & 1 & 2 & 1 & 1 & 1 & A & 5289.3617 & -0.8 \\
\hline 65 & 2 & 1 & 2 & 1 & 1 & 1 & $\mathrm{E}$ & 5295.2099 & 0.8 \\
\hline 66 & 3 & 1 & 3 & 2 & 1 & 2 & A & 7914.3435 & -0.4 \\
\hline 67 & 3 & 1 & 3 & 2 & 1 & 2 & $E$ & 7915.3411 & -0.4 \\
\hline 68 & 4 & 1 & 4 & 3 & 1 & 3 & A & 10518.0154 & 0.2 \\
\hline 69 & 4 & 1 & 4 & 3 & 1 & 3 & E & 10517.9097 & 1.1 \\
\hline 70 & 5 & 1 & 5 & 4 & 1 & 4 & A & 13096.8873 & 0.3 \\
\hline 71 & 5 & 1 & 5 & 4 & 1 & 4 & $\mathrm{E}$ & 13096.3678 & 0.5 \\
\hline 72 & 6 & 1 & 6 & 5 & 1 & 5 & A & 15649.9566 & -0.4 \\
\hline 73 & 6 & 1 & 6 & 5 & 1 & 5 & $E$ & 15649.2505 & -0.3 \\
\hline 74 & 7 & 1 & 7 & 6 & 1 & 6 & A & 18178.5328 & -0.8 \\
\hline 75 & 7 & 1 & 7 & 6 & 1 & 6 & $\mathrm{E}$ & 18177.7428 & 0.2 \\
\hline 76 & 8 & 1 & 8 & 7 & 1 & 7 & A & 20685.6584 & 0.6 \\
\hline 77 & 8 & 1 & 8 & 7 & 1 & 7 & $E$ & 20684.8352 & 0.5 \\
\hline 78 & 9 & 1 & 9 & 8 & 1 & 8 & A & 23175.3034 & -0.8 \\
\hline 79 & 9 & 1 & 9 & 8 & 1 & 8 & $E$ & 23174.4767 & -0.7 \\
\hline 80 & 2 & 1 & 1 & 1 & 1 & 1 & $E$ & 6473.1051 & -1.3 \\
\hline 81 & 2 & 1 & 1 & 1 & 1 & 0 & A & 6070.7727 & 3.6 \\
\hline
\end{tabular}




\begin{tabular}{|c|c|c|c|c|c|c|c|c|c|}
\hline 82 & 2 & 1 & 1 & 1 & 1 & 0 & $\mathrm{E}$ & 6063.5338 & -0.7 \\
\hline 83 & 3 & 1 & 2 & 2 & 1 & 1 & A & 9084.7260 & 1.4 \\
\hline 84 & 3 & 1 & 2 & 2 & 1 & 1 & E & 9081.5013 & 0.1 \\
\hline 85 & 4 & 1 & 3 & 3 & 1 & 2 & A & 12070.5088 & 0.8 \\
\hline 86 & 4 & 1 & 3 & 3 & 1 & 2 & E & 12067.5031 & -1.6 \\
\hline 87 & 5 & 1 & 4 & 4 & 1 & 3 & $\mathrm{E}$ & 15011.2473 & -0.4 \\
\hline 88 & 6 & 1 & 5 & 5 & 1 & 4 & A & 17900.5287 & 1.0 \\
\hline 89 & 6 & 1 & 5 & 5 & 1 & 4 & E & 17896.7407 & -1.0 \\
\hline 90 & 7 & 1 & 6 & 6 & 1 & 5 & A & 20709.1263 & 1.1 \\
\hline 91 & 7 & 1 & 6 & 6 & 1 & 5 & E & 20705.1687 & -0.8 \\
\hline 92 & 8 & 1 & 7 & 7 & 1 & 6 & A & 23422.1627 & 0.2 \\
\hline 93 & 2 & 1 & 2 & 1 & 1 & 0 & $\mathrm{E}$ & 4885.6365 & -0.8 \\
\hline 94 & 5 & 1 & 4 & 4 & 2 & 3 & A & 6449.9779 & 1.6 \\
\hline 95 & 5 & 1 & 4 & 4 & 2 & 3 & $\mathrm{E}$ & 6480.8444 & 1.3 \\
\hline 96 & 6 & 1 & 5 & 5 & 2 & 4 & $\mathrm{E}$ & 10236.0851 & -1.0 \\
\hline 97 & 7 & 1 & 6 & 6 & 2 & 5 & A & 14038.9782 & 4.2 \\
\hline 98 & 7 & 1 & 6 & 6 & 2 & 5 & $\mathrm{E}$ & 14043.6070 & -1.3 \\
\hline 99 & 8 & 1 & 7 & 7 & 2 & 6 & A & 17832.9375 & 5.3 \\
\hline 100 & 6 & 1 & 5 & 5 & 2 & 3 & $\mathrm{E}$ & 9138.4400 & 1.0 \\
\hline 101 & 3 & 2 & 2 & 3 & 0 & 3 & A & 14097.4756 & 1.2 \\
\hline 102 & 4 & 2 & 3 & 4 & 0 & 4 & $\mathrm{E}$ & 14350.4603 & 0.9 \\
\hline 103 & 2 & 2 & 0 & 1 & 1 & 1 & A & 16359.4958 & 2.0 \\
\hline 104 & 2 & 2 & 0 & 1 & 1 & 1 & $\mathrm{E}$ & 16470.2781 & -4.2 \\
\hline 105 & 2 & 2 & 0 & 1 & 1 & 0 & $\mathrm{E}$ & 16060.7080 & -2.4 \\
\hline 106 & 2 & 2 & 1 & 1 & 1 & 1 & $\mathrm{E}$ & 16221.6314 & 3.6 \\
\hline 107 & 2 & 2 & 1 & 1 & 1 & 0 & A & 15936.0012 & -3.1 \\
\hline 108 & 2 & 2 & 1 & 1 & 1 & 0 & $E$ & 15812.0610 & 5.1 \\
\hline 109 & 3 & 2 & 2 & 2 & 1 & 1 & A & 18385.3265 & -0.5 \\
\hline 110 & 3 & 2 & 2 & 2 & 1 & 1 & $E$ & 18309.5249 & -2.3 \\
\hline 111 & 4 & 2 & 3 & 3 & 1 & 2 & A & 20635.1714 & -1.8 \\
\hline 112 & 4 & 2 & 3 & 3 & 1 & 2 & $\mathrm{E}$ & 20597.9089 & -0.4 \\
\hline 113 & 2 & 2 & 0 & 2 & 1 & 1 & A & 9898.0195 & -1.5 \\
\hline 114 & 3 & 2 & 1 & 3 & 1 & 2 & A & 9463.0207 & -2.5 \\
\hline 115 & 3 & 2 & 1 & 3 & 1 & 2 & $\mathrm{E}$ & 9522.1047 & 1.0 \\
\hline 116 & 4 & 2 & 2 & 4 & 1 & 3 & A & 9041.8371 & 0.9 \\
\hline 117 & 4 & 2 & 2 & 4 & 1 & 3 & $\mathrm{E}$ & 9065.3363 & -0.3 \\
\hline 118 & 5 & 2 & 3 & 5 & 1 & 4 & $\mathrm{E}$ & 8758.3010 & -1.7 \\
\hline 119 & 6 & 2 & 4 & 6 & 1 & 5 & A & 8697.2803 & -0.6 \\
\hline 120 & 6 & 2 & 4 & 6 & 1 & 5 & $\mathrm{E}$ & 8700.0407 & -2.1 \\
\hline 121 & 2 & 2 & 0 & 2 & 1 & 2 & $\mathrm{E}$ & 11175.0737 & 0.6 \\
\hline 122 & 3 & 2 & 1 & 2 & 1 & 2 & A & 19719.8639 & 5.8 \\
\hline 123 & 3 & 2 & 1 & 2 & 1 & 2 & $\mathrm{E}$ & 19781.5022 & 0.1 \\
\hline 124 & 4 & 2 & 2 & 3 & 1 & 3 & $\mathrm{E}$ & 23476.8984 & 0.2 \\
\hline
\end{tabular}




\begin{tabular}{|c|c|c|c|c|c|c|c|c|c|}
\hline 125 & 3 & 2 & 1 & 2 & 1 & 1 & $\mathrm{E}$ & 18603.6069 & 2.0 \\
\hline 126 & 4 & 2 & 2 & 3 & 1 & 2 & $\mathrm{E}$ & 21132.8404 & -0.9 \\
\hline 127 & 3 & 2 & 1 & 3 & 1 & 3 & $E$ & 11866.1645 & 3.9 \\
\hline 128 & 4 & 2 & 2 & 4 & 1 & 4 & $E$ & 12958.9903 & 0.7 \\
\hline 129 & 3 & 2 & 2 & 3 & 1 & 2 & $\mathrm{E}$ & 9228.0231 & -3.0 \\
\hline 130 & 3 & 2 & 2 & 2 & 1 & 2 & $E$ & 19487.4227 & -1.8 \\
\hline 131 & 5 & 2 & 4 & 4 & 1 & 3 & A & 22691.9880 & 2.6 \\
\hline 132 & 5 & 2 & 4 & 4 & 1 & 3 & $E$ & 22671.9030 & -0.3 \\
\hline 133 & 6 & 2 & 5 & 5 & 1 & 4 & A & 24570.6764 & -2.5 \\
\hline 134 & 6 & 2 & 5 & 5 & 1 & 4 & $E$ & 24558.3027 & -0.3 \\
\hline 135 & 4 & 2 & 2 & 3 & 1 & 3 & A & 23454.8314 & -3.8 \\
\hline 136 & 4 & 2 & 3 & 4 & 1 & 3 & $E$ & 8530.4039 & -0.7 \\
\hline 137 & 2 & 2 & 1 & 2 & 1 & 2 & A & 11037.3487 & 3.1 \\
\hline 138 & 2 & 2 & 1 & 2 & 1 & 2 & $E$ & 10926.4149 & -3.7 \\
\hline 139 & 3 & 2 & 2 & 3 & 1 & 3 & A & 11643.0946 & 1.1 \\
\hline 140 & 3 & 2 & 2 & 3 & 1 & 3 & $\mathrm{E}$ & 11572.0807 & -2.3 \\
\hline 141 & 4 & 2 & 3 & 4 & 1 & 4 & A & 12459.6484 & -0.6 \\
\hline 142 & 4 & 2 & 3 & 4 & 1 & 4 & $\mathrm{E}$ & 12424.0584 & 0.8 \\
\hline 143 & 5 & 2 & 4 & 5 & 1 & 5 & A & 13490.0903 & 8.2 \\
\hline 144 & 5 & 2 & 4 & 5 & 1 & 5 & $\mathrm{E}$ & 13469.1898 & 0.7 \\
\hline 145 & 6 & 2 & 5 & 6 & 1 & 6 & A & 14733.4608 & 0.6 \\
\hline 146 & 6 & 2 & 5 & 6 & 1 & 6 & E & 14717.5854 & -0.2 \\
\hline 147 & 7 & 2 & 6 & 7 & 1 & 7 & A & 16183.1314 & 0.6 \\
\hline 148 & 8 & 2 & 7 & 8 & 1 & 8 & A & 17825.9484 & 0.9 \\
\hline 149 & 5 & 2 & 4 & 4 & 2 & 2 & $\mathrm{E}$ & 13606.5673 & 0.5 \\
\hline 150 & 5 & 2 & 3 & 4 & 2 & 3 & $E$ & 15239.1456 & -0.2 \\
\hline 151 & 3 & 2 & 2 & 2 & 2 & 1 & A & 8520.0937 & 2.0 \\
\hline 152 & 4 & 2 & 3 & 3 & 2 & 2 & A & 11334.5709 & 0.1 \\
\hline 153 & 4 & 2 & 3 & 3 & 2 & 2 & $E$ & 11369.8851 & 1.9 \\
\hline 154 & 5 & 2 & 4 & 4 & 2 & 3 & A & 14127.3195 & -0.6 \\
\hline 155 & 5 & 2 & 4 & 4 & 2 & 3 & $\mathrm{E}$ & 14141.4992 & 0.4 \\
\hline 156 & 6 & 2 & 5 & 5 & 2 & 4 & A & 16893.3337 & -1.3 \\
\hline 157 & 6 & 2 & 5 & 5 & 2 & 4 & $E$ & 16897.6463 & -1.1 \\
\hline 158 & 8 & 2 & 7 & 7 & 2 & 6 & A & 22328.4735 & -0.9 \\
\hline 159 & 3 & 2 & 1 & 2 & 2 & 0 & A & 8649.7284 & 1.6 \\
\hline 160 & 3 & 2 & 1 & 2 & 2 & 0 & $E$ & 8606.4258 & -3.2 \\
\hline 161 & 4 & 2 & 2 & 3 & 2 & 1 & A & 11649.3213 & 0.3 \\
\hline 162 & 4 & 2 & 2 & 3 & 2 & 1 & $E$ & 11610.7352 & -2.4 \\
\hline 163 & 5 & 2 & 3 & 4 & 2 & 2 & A & 14722.6074 & 0.9 \\
\hline 164 & 5 & 2 & 3 & 4 & 2 & 2 & $E$ & 14704.2125 & -1.3 \\
\hline 165 & 6 & 2 & 4 & 5 & 2 & 3 & A & 17848.0073 & -0.1 \\
\hline 166 & 6 & 2 & 4 & 5 & 2 & 3 & $E$ & 17838.4807 & -1.1 \\
\hline 167 & 7 & 2 & 5 & 6 & 2 & 4 & A & 20987.2895 & 0.1 \\
\hline
\end{tabular}




\begin{tabular}{|c|c|c|c|c|c|c|c|c|c|}
\hline 168 & 7 & 2 & 5 & 6 & 2 & 4 & $\mathrm{E}$ & 20980.5259 & -1.8 \\
\hline 169 & 8 & 2 & 6 & 7 & 2 & 5 & A & 24101.4972 & 0.8 \\
\hline 170 & 4 & 2 & 2 & 3 & 2 & 2 & $\mathrm{E}$ & 11904.8150 & -0.2 \\
\hline 171 & 6 & 2 & 5 & 5 & 2 & 3 & $\mathrm{E}$ & 15800.0006 & 0.3 \\
\hline 172 & 3 & 3 & 1 & 3 & 2 & 2 & A & 17438.4762 & -1.3 \\
\hline 173 & 3 & 3 & 1 & 3 & 2 & 2 & $\mathrm{E}$ & 17310.8703 & -3.8 \\
\hline 174 & 4 & 3 & 2 & 4 & 2 & 3 & A & 17524.7161 & 1.3 \\
\hline 175 & 5 & 3 & 3 & 5 & 2 & 4 & A & 17693.1838 & 0.1 \\
\hline 176 & 5 & 3 & 3 & 5 & 2 & 4 & $\mathrm{E}$ & 17535.0465 & 1.6 \\
\hline 177 & 6 & 3 & 4 & 6 & 2 & 5 & A & 17975.8525 & 0.8 \\
\hline 178 & 6 & 3 & 4 & 6 & 2 & 5 & $\mathrm{E}$ & 17847.2415 & 6.1 \\
\hline 179 & 7 & 3 & 5 & 7 & 2 & 6 & A & 18403.6805 & -2.6 \\
\hline 180 & 3 & 3 & 0 & 3 & 2 & 1 & A & 17277.7841 & 0.5 \\
\hline 181 & 4 & 3 & 1 & 4 & 2 & 2 & A & 17059.5865 & 2.8 \\
\hline 182 & 5 & 3 & 2 & 5 & 2 & 3 & A & 16668.5118 & 3.1 \\
\hline 183 & 3 & 3 & 0 & 4 & 2 & 3 & A & 6105.6321 & -1.5 \\
\hline 184 & 4 & 3 & 2 & 4 & 2 & 2 & $\mathrm{E}$ & 16830.7227 & -2.8 \\
\hline 185 & 5 & 3 & 3 & 5 & 2 & 3 & $\mathrm{E}$ & 16437.4001 & 2.2 \\
\hline 186 & 4 & 3 & 1 & 3 & 3 & 0 & A & 11431.1225 & 1.5 \\
\hline 187 & 5 & 3 & 2 & 4 & 3 & 1 & $\mathrm{E}$ & 14312.3298 & -6.4 \\
\hline 188 & 5 & 3 & 3 & 4 & 3 & 2 & A & 14295.7874 & -1.6 \\
\hline 189 & 5 & 3 & 3 & 4 & 3 & 2 & $\mathrm{E}$ & 14310.8898 & 3.6 \\
\hline 190 & 6 & 3 & 4 & 5 & 3 & 3 & A & 17176.0016 & -1.4 \\
\hline 191 & 6 & 3 & 4 & 5 & 3 & 3 & $\mathrm{E}$ & 17209.8412 & 3.4 \\
\hline 192 & 7 & 3 & 5 & 6 & 3 & 4 & A & 20056.0344 & -1.3 \\
\hline 193 & 7 & 3 & 5 & 6 & 3 & 4 & $E$ & 20100.9648 & 0.1 \\
\hline 194 & 8 & 3 & 6 & 7 & 3 & 5 & A & 22928.7757 & -1.6 \\
\hline 195 & 6 & 3 & 3 & 5 & 3 & 2 & A & 17269.5911 & -1.5 \\
\hline 196 & 7 & 3 & 4 & 6 & 3 & 3 & A & 20260.1544 & -1.2 \\
\hline 197 & 7 & 3 & 4 & 6 & 3 & 3 & $E$ & 20209.1816 & -4.9 \\
\hline 198 & 6 & 3 & 3 & 6 & 2 & 4 & A & 16090.0959 & 2.0 \\
\hline 199 & 7 & 3 & 4 & 7 & 2 & 5 & A & 15362.9556 & -4.6 \\
\hline 200 & 4 & 4 & 0 & 4 & 3 & 1 & A & 24319.3982 & -1.9 \\
\hline 201 & 5 & 4 & 1 & 5 & 3 & 2 & A & 24261.3925 & 0.3 \\
\hline 202 & 5 & 4 & 2 & 5 & 3 & 3 & A & 24308.4985 & 0.7 \\
\hline 203 & 5 & 4 & 1 & 4 & 4 & 0 & A & 14273.5220 & -1.6 \\
\hline 204 & 6 & 4 & 2 & 5 & 4 & 1 & A & 17153.4315 & -3.4 \\
\hline 205 & 5 & 4 & 2 & 4 & 4 & 1 & A & 14272.9220 & -0.2 \\
\hline 206 & 5 & 4 & 2 & 4 & 4 & 1 & $\mathrm{E}$ & 14271.6242 & 4.1 \\
\hline 207 & 6 & 4 & 3 & 5 & 4 & 2 & A & 17150.7390 & -4.3 \\
\hline 208 & 6 & 4 & 3 & 5 & 4 & 2 & $E$ & 17150.2470 & 5.2 \\
\hline 209 & 7 & 4 & 4 & 6 & 4 & 3 & A & 20039.9471 & -4.7 \\
\hline 210 & 7 & 4 & 4 & 6 & 4 & 3 & $\mathrm{E}$ & 20042.2998 & 5.8 \\
\hline
\end{tabular}




\begin{tabular}{rrrrrrrrrr}
\hline $\mathbf{2 1 1}$ & 6 & 5 & 2 & 5 & 5 & 1 & $\mathrm{E}$ & 17120.4041 & 5.0 \\
$\mathbf{2 1 2}$ & 6 & 5 & 1 & 5 & 5 & 0 & $\mathrm{E}$ & 17119.4836 & -4.3 \\
$\mathbf{2 1 3}$ & 7 & 5 & 3 & 6 & 5 & 2 & $\mathrm{E}$ & 19994.6654 & 5.2 \\
\hline
\end{tabular}

Table S7a: Observed frequencies $v_{o b s}$ of 43 rotational transitions of the ${ }^{18} \mathrm{O}(1)$ isotopologue (for atom numbering see Figure 1) of anti-2AF with $v_{o b s}-v_{c a l c}$ values obtained from a fit with the program $X I A M$.

\begin{tabular}{rrrrrrrrrrr}
\hline No. & $\boldsymbol{J}$ & $\boldsymbol{K}_{\boldsymbol{a}}$ & $\boldsymbol{K}_{\boldsymbol{c}}$ & $\boldsymbol{J}$ & $\boldsymbol{K}_{\boldsymbol{a}}$ & $\boldsymbol{K}_{\boldsymbol{c}}$ & Species & $\begin{array}{c}\mathbf{V}_{\text {obs }} \\
\text { [MHz] }\end{array}$ & $\begin{array}{c}\mathbf{V}_{\text {obs }}-\mathbf{V}_{\text {calc }} \\
{[\mathbf{k H z}]}\end{array}$ \\
\hline $\mathbf{1}$ & 2 & 0 & 2 & 1 & 0 & 1 & $\mathrm{~A}$ & 5657.3723 & 1.9 \\
$\mathbf{2}$ & 2 & 0 & 2 & 1 & 0 & 1 & $\mathrm{E}$ & 5657.0584 & -2.7 \\
$\mathbf{3}$ & 3 & 0 & 3 & 2 & 0 & 2 & $\mathrm{~A}$ & 8396.0266 & 0.0 \\
$\mathbf{4}$ & 3 & 0 & 3 & 2 & 0 & 2 & $\mathrm{E}$ & 8395.6467 & 2.9 \\
$\mathbf{5}$ & 5 & 0 & 5 & 4 & 0 & 4 & $\mathrm{~A}$ & 13576.2866 & -0.8 \\
$\mathbf{6}$ & 5 & 0 & 5 & 4 & 0 & 4 & $\mathrm{E}$ & 13575.9423 & -0.4 \\
$\mathbf{7}$ & 6 & 0 & 6 & 5 & 0 & 5 & $\mathrm{~A}$ & 16034.5987 & -1.5 \\
$\mathbf{8}$ & 6 & 0 & 6 & 5 & 0 & 5 & $\mathrm{E}$ & 16034.3126 & 0.9 \\
$\mathbf{9}$ & 7 & 0 & 7 & 6 & 0 & 6 & $\mathrm{~A}$ & 18448.5970 & -1.8 \\
$\mathbf{1 0}$ & 7 & 0 & 7 & 6 & 0 & 6 & $\mathrm{E}$ & 18448.3542 & 1.5 \\
$\mathbf{1 1}$ & 4 & 0 & 4 & 3 & 1 & 3 & $\mathrm{~A}$ & 8761.3007 & 4.2 \\
$\mathbf{1 2}$ & 4 & 0 & 4 & 3 & 1 & 3 & $\mathrm{E}$ & 8760.9132 & 0.2 \\
$\mathbf{1 3}$ & 5 & 0 & 5 & 4 & 1 & 4 & $\mathrm{~A}$ & 11826.5765 & -5.3 \\
$\mathbf{1 4}$ & 5 & 0 & 5 & 4 & 1 & 4 & $\mathrm{E}$ & 11826.0664 & 0.0 \\
$\mathbf{1 5}$ & 6 & 0 & 6 & 5 & 1 & 5 & $\mathrm{~A}$ & 14778.3314 & -2.0 \\
$\mathbf{1 6}$ & 6 & 0 & 6 & 5 & 1 & 5 & $\mathrm{E}$ & 14777.7877 & 0.1 \\
$\mathbf{1 7}$ & 2 & 1 & 2 & 1 & 0 & 1 & $\mathrm{~A}$ & 8417.3928 & -2.8 \\
$\mathbf{1 8}$ & 2 & 1 & 2 & 1 & 0 & 1 & $\mathrm{E}$ & 8416.8085 & -2.4 \\
$\mathbf{1 9}$ & 3 & 1 & 3 & 2 & 0 & 2 & $\mathrm{~A}$ & 10672.0189 & -1.8 \\
$\mathbf{2 0}$ & 3 & 1 & 3 & 2 & 0 & 2 & $\mathrm{E}$ & 10671.6338 & 0.2 \\
$\mathbf{2 1}$ & 4 & 1 & 4 & 3 & 0 & 3 & $\mathrm{~A}$ & 12786.9954 & -0.8 \\
$\mathbf{2 2}$ & 4 & 1 & 4 & 3 & 0 & 3 & $\mathrm{E}$ & 12786.7805 & 1.4 \\
$\mathbf{2 3}$ & 5 & 1 & 5 & 4 & 0 & 4 & $\mathrm{~A}$ & 14832.5535 & -0.7 \\
$\mathbf{2 4}$ & 5 & 1 & 5 & 4 & 0 & 4 & $\mathrm{E}$ & 14832.4678 & 1.0 \\
$\mathbf{2 5}$ & 6 & 1 & 6 & 5 & 0 & 5 & $\mathrm{~A}$ & 16883.1857 & 4.7 \\
$\mathbf{2 6}$ & 6 & 1 & 6 & 5 & 0 & 5 & $\mathrm{E}$ & 16883.1624 & 1.5 \\
$\mathbf{2 7}$ & 3 & 1 & 3 & 2 & 1 & 2 & $\mathrm{~A}$ & 7911.9945 & -1.0 \\
$\mathbf{2 8}$ & 3 & 1 & 3 & 2 & 1 & 2 & $\mathrm{E}$ & 7911.8856 & 1.8 \\
$\mathbf{2 9}$ & 4 & 1 & 4 & 3 & 1 & 3 & $\mathrm{~A}$ & 10511.0010 & -1.1 \\
$\mathbf{3 0}$ & 4 & 1 & 4 & 3 & 1 & 3 & $\mathrm{E}$ & 10510.7895 & 0.2 \\
$\mathbf{3 1}$ & 5 & 1 & 5 & 4 & 1 & 4 & $\mathrm{~A}$ & 13082.8458 & -2.9 \\
$\mathbf{3 2}$ & 5 & 1 & 5 & 4 & 1 & 4 & $\mathrm{E}$ & 13082.5906 & 0.1 \\
\hline & & & & & & & &
\end{tabular}




\begin{tabular}{llllllllrr}
\hline $\mathbf{3 3}$ & 6 & 1 & 6 & 5 & 1 & 5 & $\mathrm{~A}$ & 15626.9125 & -1.7 \\
$\mathbf{3 4}$ & 3 & 1 & 2 & 2 & 1 & 1 & $\mathrm{~A}$ & 9124.3269 & 0.8 \\
$\mathbf{3 5}$ & 3 & 1 & 2 & 2 & 1 & 1 & $\mathrm{E}$ & 9123.5838 & -0.1 \\
$\mathbf{3 6}$ & 4 & 1 & 3 & 3 & 1 & 2 & $\mathrm{~A}$ & 12117.7003 & 0.3 \\
$\mathbf{3 7}$ & 4 & 1 & 3 & 3 & 1 & 2 & $\mathrm{E}$ & 12116.8017 & 1.9 \\
$\mathbf{3 8}$ & 5 & 1 & 4 & 4 & 1 & 3 & $\mathrm{~A}$ & 15063.5009 & 1.5 \\
$\mathbf{3 9}$ & 5 & 1 & 4 & 4 & 1 & 3 & $\mathrm{E}$ & 15062.4353 & 0.4 \\
$\mathbf{4 0}$ & 6 & 1 & 5 & 5 & 1 & 4 & $\mathrm{~A}$ & 17942.4882 & 0.9 \\
$\mathbf{4 1}$ & 6 & 1 & 5 & 5 & 1 & 4 & $\mathrm{E}$ & 17941.3106 & 0.2 \\
$\mathbf{4 2}$ & 7 & 1 & 6 & 6 & 1 & 5 & $\mathrm{~A}$ & 20733.0734 & 1.2 \\
$\mathbf{4 3}$ & 7 & 1 & 6 & 6 & 1 & 5 & $\mathrm{E}$ & 20731.8626 & -1.5 \\
\hline
\end{tabular}

Table S7b: Observed frequencies $v_{\text {obs }}$ of 28 rotational transitions of the ${ }^{13} \mathrm{C}(2)$ isotopologue (for atom numbering see Figure 1) of anti-2AF with $v_{o b s}-v_{c a l c}$ values obtained from a fit with the program XIAM.

\begin{tabular}{|c|c|c|c|c|c|c|c|c|c|}
\hline No. - & \multicolumn{3}{|c|}{ upper level } & $\begin{array}{l}J \\
\text { lou }\end{array}$ & $\frac{K_{a}}{\text { er le }}$ & $\frac{\boldsymbol{K}_{c}}{\text { lel }}$ & \multirow{2}{*}{$\begin{array}{c}\text { Species } \\
\mathrm{A}\end{array}$} & \multirow{2}{*}{$\begin{array}{c}\begin{array}{c}\text { Vobs } \\
\text { [MHz] }\end{array} \\
15785.2588\end{array}$} & \multirow{2}{*}{$\begin{array}{r}\begin{array}{c}\mathbf{V}_{\text {obs }}-\mathbf{V}_{\text {calc }} \\
{[\mathbf{k H z}]}\end{array} \\
-2.4\end{array}$} \\
\hline 1 & 2 & 2 & 1 & 1 & 1 & 0 & & & \\
\hline 2 & 2 & 2 & 1 & 1 & 1 & 0 & E & 15764.4411 & -4.2 \\
\hline 3 & 3 & 2 & 2 & 2 & 1 & 1 & A & 18246.4378 & 0.7 \\
\hline 4 & 3 & 2 & 2 & 2 & 1 & 1 & E & 18239.2556 & 3.0 \\
\hline 5 & 2 & 2 & 1 & 2 & 1 & 2 & A & 10862.8990 & 1.6 \\
\hline 6 & 2 & 2 & 1 & 2 & 1 & 2 & E & 10842.9475 & -0.7 \\
\hline 7 & 3 & 2 & 2 & 3 & 1 & 3 & A & 11485.2101 & 2.9 \\
\hline 8 & 3 & 2 & 2 & 3 & 1 & 3 & E & 11478.1 & -2.4 \\
\hline 9 & 4 & 2 & 3 & 4 & 1 & 4 & A & 12324.3086 & 2.4 \\
\hline 10 & 4 & 2 & 3 & 4 & 1 & 4 & E & 12320.5647 & -3.4 \\
\hline 11 & 3 & 2 & 1 & 2 & 1 & 2 & A & 19622.8398 & 1.7 \\
\hline 12 & 3 & 2 & 1 & 2 & 1 & 2 & E & 19626.7677 & -1.1 \\
\hline 13 & 4 & 2 & 2 & 3 & 1 & 3 & $E$ & 23415.4907 & 0.1 \\
\hline 14 & 3 & 3 & 0 & 3 & 2 & 1 & A & 16951.8222 & 0.4 \\
\hline 15 & 3 & 3 & 0 & 3 & 2 & 1 & E & 16991.2573 & 1.2 \\
\hline 16 & 4 & 3 & 1 & 4 & 2 & 2 & A & 16719.4443 & -4.0 \\
\hline 17 & 4 & 3 & 1 & 4 & 2 & 2 & E & 631 & -1.9 \\
\hline 18 & 5 & 3 & 2 & 5 & 2 & 3 & A & 563 & 1.0 \\
\hline 19 & 5 & 3 & 2 & 5 & 2 & 3 & E & 1.7145 & -1.7 \\
\hline 20 & 6 & 3 & 3 & 6 & 2 & 4 & E & 3.9159 & 2.0 \\
\hline 21 & 3 & 3 & 1 & 3 & 2 & 2 & A & 17123.8146 & 3.4 \\
\hline 22 & 3 & 3 & 1 & 3 & 2 & 2 & E & 17080.6915 & 0.9 \\
\hline 23 & 4 & 3 & 2 & 4 & 2 & 3 & A & 17216.0209 & 0.8 \\
\hline 24 & 5 & 3 & 3 & 5 & 2 & 4 & A & 17395.8086 & 0.1 \\
\hline
\end{tabular}




\begin{tabular}{rrrrrrrrrr}
\hline $\mathbf{2 5}$ & 6 & 3 & 4 & 6 & 2 & 5 & A & 17696.7624 & -2.2 \\
$\mathbf{2 6}$ & 6 & 3 & 4 & 6 & 2 & 5 & E & 17681.9669 & 2.4 \\
$\mathbf{2 7}$ & 4 & 4 & 0 & 4 & 3 & 1 & A & 23871.8529 & -2.6 \\
$\mathbf{2 8}$ & 4 & 4 & 1 & 4 & 3 & 2 & A & 23885.2484 & 1.7 \\
\hline
\end{tabular}

Table S7c: Observed frequencies $v_{\text {obs }}$ of 80 rotational transitions of the ${ }^{13} \mathrm{C}(3)$ isotopologue (for atom numbering see Figure 1) of anti-2AF with $v_{o b s}-v_{\text {calc }}$ values obtained from a fit with the program XIAM.

\begin{tabular}{|c|c|c|c|c|c|c|c|c|c|}
\hline No. - & $\frac{J}{\text { upp }}$ & upper level & $\frac{K_{c}}{\text { el }}$ & $\begin{array}{l}J \\
\text { Ion }\end{array}$ & $\frac{K_{a}}{\mathrm{rlel}}$ & $\frac{K_{c}}{\mathrm{el}}$ & pecies & $\begin{array}{c}\text { Vobs } \\
{[\mathrm{MHz}]}\end{array}$ & $\begin{array}{c}V_{\text {obs }}-V_{\text {calc }} \\
{[\mathrm{kHz}]}\end{array}$ \\
\hline 1 & 2 & 0 & 2 & 1 & 0 & 1 & A & 5664.9958 & -0.9 \\
\hline 2 & 2 & 0 & 2 & 1 & 0 & 1 & $E$ & 5664.6893 & -1.8 \\
\hline 3 & 3 & 0 & 3 & 2 & 0 & 2 & A & 8409.1316 & 0.0 \\
\hline 4 & 3 & 0 & 3 & 2 & 0 & 2 & $E$ & 8408.7507 & -0.7 \\
\hline 5 & 4 & 0 & 4 & 3 & 0 & 3 & A & 11057.4015 & -0.5 \\
\hline 6 & 4 & 0 & 4 & 3 & 0 & 3 & $E$ & 11057.0137 & 0.1 \\
\hline 7 & 6 & 0 & 6 & 5 & 0 & 5 & A & 16070.4759 & -0.4 \\
\hline 8 & 6 & 0 & 6 & 5 & 0 & 5 & $E$ & 1607 & 0.3 \\
\hline 9 & 7 & 0 & 7 & 6 & 0 & 6 & A & $184{ }^{\circ}$ & -0.1 \\
\hline 10 & 7 & 0 & 7 & 6 & 0 & 6 & $E$ & 1849 & -0.3 \\
\hline 11 & 8 & 0 & 8 & 7 & 0 & 7 & A & 2089 & -0.2 \\
\hline 12 & 8 & 0 & 8 & 7 & 0 & 7 & $E$ & 2089 & 0.9 \\
\hline 13 & 9 & 0 & 9 & 8 & 0 & 8 & A & 23308 & -0.3 \\
\hline 14 & 9 & 0 & 9 & 8 & 0 & 8 & $E$ & 23308 & 0.5 \\
\hline 15 & 4 & 0 & 4 & 3 & 1 & 3 & A & 8745.8217 & 0.1 \\
\hline 16 & 4 & 0 & 4 & 3 & 1 & 3 & $E$ & 8745.4777 & -1.1 \\
\hline 17 & 5 & 0 & 5 & 4 & 1 & 4 & A & 11820.2686 & 2.6 \\
\hline 18 & 5 & 0 & 5 & 4 & 1 & 4 & $E$ & 11819.7800 & -1.4 \\
\hline 19 & 6 & 0 & 6 & 5 & 1 & 5 & A & 14783.3397 & 0.2 \\
\hline 20 & 6 & 0 & 6 & 5 & 1 & 5 & $E$ & 14782.8153 & 0.5 \\
\hline 21 & 7 & 0 & 7 & 6 & 1 & 6 & A & 17617.1637 & 1.1 \\
\hline 22 & 7 & 0 & 7 & 6 & 1 & 6 & $E$ & 17616.6609 & 0.0 \\
\hline 23 & 8 & 0 & 8 & 7 & 1 & 7 & A & 20333.4358 & 0.7 \\
\hline 24 & 8 & 0 & 8 & 7 & 1 & 7 & $E$ & 20332.9866 & 1.9 \\
\hline 25 & 9 & 0 & 9 & 8 & 1 & 8 & A & 22957.6632 & 1.0 \\
\hline 26 & 9 & 0 & 9 & 8 & 1 & 8 & E & 22957.2677 & -0.5 \\
\hline 27 & 3 & 1 & 3 & 2 & 0 & 2 & A & 10720.7189 & 6.9 \\
\hline 28 & 3 & 1 & 3 & 2 & 0 & 2 & E & 10720.2862 & 0.0 \\
\hline 29 & 4 & 1 & 4 & 3 & 0 & 3 & A & 12841.5100 & 2.7 \\
\hline 30 & 4 & 1 & 4 & 3 & 0 & 3 & $E$ & 12841.2555 & 0.8 \\
\hline 31 & 5 & 1 & 5 & 4 & 0 & 4 & A & 14891.5082 & 0.1 \\
\hline
\end{tabular}




\begin{tabular}{|c|c|c|c|c|c|c|c|c|c|}
\hline 32 & 5 & 1 & 5 & 4 & 0 & 4 & $E$ & 14891.3864 & -3.0 \\
\hline 33 & 6 & 1 & 6 & 5 & 0 & 5 & A & 16944.5484 & -3.0 \\
\hline 34 & 6 & 1 & 6 & 5 & 0 & 5 & $\mathrm{E}$ & 16944.5063 & 0.3 \\
\hline 35 & 7 & 1 & 7 & 6 & 0 & 6 & A & 19055.8835 & -2.4 \\
\hline 36 & 7 & 1 & 7 & 6 & 0 & 6 & E & 19055.8555 & 0.9 \\
\hline 37 & 8 & 1 & 8 & 7 & 0 & 7 & A & 21248.8905 & -1.1 \\
\hline 38 & 8 & 1 & 8 & 7 & 0 & 7 & E & 21248.8366 & 0.0 \\
\hline 39 & 3 & 1 & 2 & 2 & 1 & 1 & A & 9132.2924 & -0.3 \\
\hline 40 & 3 & 1 & 2 & 2 & 1 & 1 & E & 9131.5524 & 1.1 \\
\hline 41 & 4 & 1 & 3 & 3 & 1 & 2 & A & 12129.3162 & 0.4 \\
\hline 42 & 4 & 1 & 3 & 3 & 1 & 2 & $\mathrm{E}$ & 12128.4243 & 0.9 \\
\hline 43 & 5 & 1 & 4 & 4 & 1 & 3 & A & 15079.8239 & 0.1 \\
\hline 44 & 5 & 1 & 4 & 4 & 1 & 3 & $E$ & 15078.7691 & 0.1 \\
\hline 45 & 6 & 1 & 5 & 5 & 1 & 4 & A & 17965.0541 & -0.2 \\
\hline 46 & 6 & 1 & 5 & 5 & 1 & 4 & $E$ & 17963.8856 & 0.7 \\
\hline 47 & 7 & 1 & 6 & 6 & 1 & 5 & A & 20763.8525 & 0.7 \\
\hline 48 & 7 & 1 & 6 & 6 & 1 & 5 & $\mathrm{E}$ & 20762.6427 & -2.5 \\
\hline 49 & 8 & 1 & 7 & 7 & 1 & 6 & A & 23457.1748 & -0.2 \\
\hline 50 & 8 & 1 & 7 & 7 & 1 & 6 & $\mathrm{E}$ & 23456.0152 & 0.3 \\
\hline 51 & 4 & 1 & 4 & 3 & 1 & 3 & A & 10529.9266 & -0.3 \\
\hline 52 & 4 & 1 & 4 & 3 & 1 & 3 & $E$ & 10529.7191 & -0.8 \\
\hline 53 & 5 & 1 & 5 & 4 & 1 & 4 & A & 13107.4013 & -1.5 \\
\hline 54 & 5 & 1 & 5 & 4 & 1 & 4 & $E$ & 13107.1479 & -0.4 \\
\hline 55 & 6 & 1 & 6 & 5 & 1 & 5 & A & 15657.4140 & -0.5 \\
\hline 56 & 6 & 1 & 6 & 5 & 1 & 5 & E & 15657.1385 & -0.6 \\
\hline 57 & 7 & 1 & 7 & 6 & 1 & 6 & A & 18181.8110 & 0.2 \\
\hline 58 & 7 & 1 & 7 & 6 & 1 & 6 & E & 18181.5308 & 0.5 \\
\hline 59 & 8 & 1 & 8 & 7 & 1 & 7 & A & 20684.2434 & -0.1 \\
\hline 60 & 8 & 1 & 8 & 7 & 1 & 7 & $E$ & 20683.9669 & -0.3 \\
\hline 61 & 2 & 2 & 1 & 1 & 1 & 0 & A & 15589.3839 & -6.3 \\
\hline 62 & 4 & 2 & 3 & 3 & 1 & 2 & A & 20278.8057 & 5.1 \\
\hline 63 & 2 & 2 & 0 & 1 & 1 & 1 & A & 16028.3708 & 1.5 \\
\hline 64 & 3 & 2 & 1 & 2 & 1 & 2 & A & 19424.2965 & -1.0 \\
\hline 65 & 4 & 2 & 2 & 3 & 2 & 1 & A & 11718.7207 & -0.9 \\
\hline 66 & 4 & 2 & 2 & 3 & 2 & 1 & $E$ & 11714.3895 & 0.7 \\
\hline 67 & 6 & 2 & 4 & 5 & 2 & 3 & A & 17971.0731 & -1.5 \\
\hline 68 & 6 & 2 & 4 & 5 & 2 & 3 & $\mathrm{E}$ & 17969.3745 & -0.7 \\
\hline 69 & 7 & 2 & 5 & 6 & 2 & 4 & A & 21128.1645 & 0.1 \\
\hline 70 & 7 & 2 & 5 & 6 & 2 & 4 & $E$ & 21126.4230 & 0.7 \\
\hline 71 & 4 & 2 & 3 & 3 & 2 & 2 & A & 11374.0416 & -0.3 \\
\hline 72 & 4 & 2 & 3 & 3 & 2 & 2 & E & 11377.0606 & 1.3 \\
\hline 73 & 5 & 2 & 4 & 4 & 2 & 3 & A & 14172.5412 & -2.0 \\
\hline 74 & 5 & 2 & 4 & 4 & 2 & 3 & $E$ & 14172.9258 & 0.3 \\
\hline
\end{tabular}




\begin{tabular}{rrrrrrrrrr}
\hline $\mathbf{7 5}$ & 5 & 3 & 3 & 4 & 3 & 2 & $\mathrm{~A}$ & 14356.8508 & 1.2 \\
$\mathbf{7 6}$ & 5 & 3 & 3 & 4 & 3 & 2 & $\mathrm{E}$ & 14370.0966 & 1.9 \\
$\mathbf{7 7}$ & 6 & 3 & 4 & 5 & 3 & 3 & $\mathrm{~A}$ & 17249.8454 & -0.9 \\
$\mathbf{7 8}$ & 6 & 3 & 4 & 5 & 3 & 3 & $\mathrm{E}$ & 17263.4949 & 0.0 \\
$\mathbf{7 9}$ & 7 & 3 & 5 & 6 & 3 & 4 & $\mathrm{~A}$ & 20141.2861 & 0.7 \\
$\mathbf{8 0}$ & 7 & 3 & 5 & 6 & 3 & 4 & $\mathrm{E}$ & 20147.3187 & -1.3 \\
\hline
\end{tabular}

Table S7d: Observed frequencies $v_{\text {obs }}$ of 81 rotational transitions of the ${ }^{13} \mathrm{C}(4)$ isotopologue (for atom numbering see Figure 1) of anti-2AF with $v_{o b s}-v_{c a l c}$ values obtained from a fit with the program XIAM.

\begin{tabular}{|c|c|c|c|c|c|c|c|c|c|}
\hline No. & \multicolumn{3}{|c|}{ upper level } & \multicolumn{3}{|c|}{ lower level } & \multirow{2}{*}{$\begin{array}{c}\text { Species } \\
\text { A }\end{array}$} & \multirow{2}{*}{ 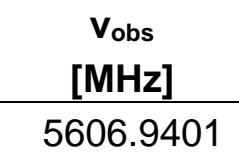 } & \multirow{2}{*}{$\begin{array}{r}\begin{array}{c}\mathbf{V}_{\text {obs }}-\mathbf{V}_{\text {calc }} \\
{[\mathrm{kHz}]}\end{array} \\
0.4\end{array}$} \\
\hline 1 & 2 & 0 & 2 & 1 & 0 & 1 & & & \\
\hline 2 & 2 & 0 & 2 & 1 & 0 & 1 & $\mathrm{E}$ & 5606.6410 & -0.6 \\
\hline 3 & 3 & 0 & 3 & 2 & 0 & 2 & & 8328.6894 & -3.2 \\
\hline 4 & 3 & 0 & 3 & 2 & 0 & 2 & & 8328.3 & -1.8 \\
\hline 5 & 4 & 0 & 4 & 3 & 0 & 3 & & 10960. & 0.2 \\
\hline 6 & 4 & 0 & 4 & 3 & 0 & 3 & & 75 & -0.8 \\
\hline 7 & 5 & 0 & 5 & 4 & 0 & 4 & & 81 & 1.5 \\
\hline 8 & 5 & 0 & 5 & 4 & 0 & 4 & & 43 & 0.3 \\
\hline 9 & 6 & 0 & 6 & 5 & 0 & 5 & & 5952 & -0.7 \\
\hline 10 & 6 & c & 6 & 5 & 0 & 5 & & 5952. & -0.1 \\
\hline 11 & 8 & 0 & 8 & 7 & 0 & 7 & & 37 & 5.1 \\
\hline 12 & 8 & 0 & 8 & 7 & 0 & 7 & & 29 & -5.4 \\
\hline 13 & 9 & c & 9 & 8 & 0 & 8 & & 52 & -0.7 \\
\hline 14 & 9 & 0 & 9 & 8 & 0 & 8 & & 72 & 0.0 \\
\hline 15 & 6 & 0 & 6 & 5 & 1 & 5 & & 204 & -0.7 \\
\hline 16 & 6 & 0 & 6 & 5 & 1 & 5 & & 1457 & -0.2 \\
\hline 17 & 7 & 0 & 7 & 6 & 1 & 6 & & 17410 & 0.2 \\
\hline 18 & 7 & 0 & 7 & 6 & 1 & 6 & & 1740 & 0.3 \\
\hline 19 & 8 & 0 & 8 & 7 & 1 & 7 & & 2012 & 0.8 \\
\hline 20 & 8 & 0 & 8 & 7 & 1 & 7 & & 20125 & -1.1 \\
\hline 21 & 9 & 0 & 9 & 8 & 1 & 8 & A & 22747.2323 & -1.3 \\
\hline 22 & 9 & 0 & 9 & 8 & 1 & 8 & & 22746 & 2.3 \\
\hline 23 & 4 & 1 & 4 & 3 & 0 & 3 & A & 12837.1 & -2.5 \\
\hline 24 & 4 & 1 & 4 & 3 & 0 & 3 & & 12836.86 & 0.1 \\
\hline 25 & 5 & 1 & 5 & 4 & 0 & 4 & & 14871.9584 & 3.0 \\
\hline 26 & 5 & 1 & 5 & 4 & 0 & 4 & $\mathrm{E}$ & 14871.8286 & 0.7 \\
\hline 27 & 6 & 1 & 6 & 5 & 0 & 5 & A & 16903.0961 & -2.1 \\
\hline 28 & 6 & 1 & 6 & 5 & 0 & 5 & $\mathrm{E}$ & 16903.0530 & 0.9 \\
\hline 29 & 7 & 1 & 7 & 6 & 0 & 6 & A & 18985.9587 & -0.3 \\
\hline
\end{tabular}




\begin{tabular}{|c|c|c|c|c|c|c|c|c|c|}
\hline 30 & 7 & 1 & 7 & 6 & 0 & 6 & $\mathrm{E}$ & 18985.9359 & 0.8 \\
\hline 31 & 8 & 1 & 8 & 7 & 0 & 7 & A & 21147.0106 & -0.3 \\
\hline 32 & 8 & 1 & 8 & 7 & 0 & 7 & $E$ & 21146.9686 & 0.3 \\
\hline 33 & 9 & 1 & 9 & 8 & 0 & 8 & A & 23386.1890 & -0.1 \\
\hline 34 & 9 & 1 & 9 & 8 & 0 & 8 & $E$ & 23386.1099 & -0.2 \\
\hline 35 & 2 & 1 & 1 & 1 & 1 & 0 & A & 6030.8732 & 0.7 \\
\hline 36 & 2 & 1 & 1 & 1 & 1 & 0 & $E$ & 6030.0264 & 1.3 \\
\hline 37 & 3 & 1 & 2 & 2 & 1 & 1 & A & 9024.5153 & 0.3 \\
\hline 38 & 3 & 1 & 2 & 2 & 1 & 1 & $E$ & 9023.7860 & -0.4 \\
\hline 39 & 4 & 1 & 3 & 3 & 1 & 2 & A & 11989.4657 & -0.5 \\
\hline 40 & 4 & 1 & 3 & 3 & 1 & 2 & $E$ & 11988.5946 & -0.8 \\
\hline 41 & 5 & 1 & 4 & 4 & 1 & 3 & A & 14911.9433 & -0.7 \\
\hline 42 & 5 & 1 & 4 & 4 & 1 & 3 & E & 14910.9122 & -1.1 \\
\hline 43 & 6 & 1 & 5 & 5 & 1 & 4 & A & 17774.9689 & -0.2 \\
\hline 44 & 7 & 1 & 6 & 6 & 1 & 5 & A & 20559.1380 & 0.8 \\
\hline 45 & 3 & 1 & 3 & 2 & 1 & 2 & A & 7854.4556 & 0.0 \\
\hline 46 & 3 & 1 & 3 & 2 & 1 & 2 & $E$ & 7854.3665 & 0.7 \\
\hline 47 & 4 & 1 & 4 & 3 & 1 & 3 & A & 10437.6671 & 1.4 \\
\hline 48 & 4 & 1 & 4 & 3 & 1 & 3 & E & 10437.4660 & -0.6 \\
\hline 49 & 5 & 1 & 5 & 4 & 1 & 4 & A & 12995.7955 & -0.2 \\
\hline 50 & 5 & 1 & 5 & 4 & 1 & 4 & $E$ & 12995.5470 & -0.2 \\
\hline 51 & 6 & 1 & 6 & 5 & 1 & 5 & A & 15527.9196 & 0.2 \\
\hline 52 & 6 & 1 & 6 & 5 & 1 & 5 & $E$ & 15527.6481 & -0.1 \\
\hline 53 & 9 & 1 & 9 & 8 & 1 & 8 & $\mathrm{E}$ & 22990.0903 & 0.2 \\
\hline 54 & 4 & 2 & 3 & 3 & 2 & 2 & A & 11254.5396 & 0.7 \\
\hline 55 & 4 & 2 & 3 & 3 & 2 & 2 & $\mathrm{E}$ & 11257.9266 & 0.7 \\
\hline 56 & 5 & 2 & 4 & 4 & 2 & 3 & A & 14026.6205 & 0.3 \\
\hline 57 & 5 & 2 & 4 & 4 & 2 & 3 & $E$ & 14027.1331 & -0.1 \\
\hline 58 & 6 & 2 & 5 & 5 & 2 & 4 & A & 16771.5546 & 1.3 \\
\hline 59 & 6 & 2 & 5 & 5 & 2 & 4 & $E$ & 16771.2363 & -0.4 \\
\hline 60 & 7 & 2 & 6 & 6 & 2 & 5 & A & 19484.8911 & 1.8 \\
\hline 61 & 7 & 2 & 6 & 6 & 2 & 5 & $E$ & 19484.2488 & -1.0 \\
\hline 62 & 4 & 2 & 2 & 3 & 2 & 1 & A & 11573.9880 & 3.1 \\
\hline 63 & 4 & 2 & 2 & 3 & 2 & 1 & E & 11569.3239 & -1.6 \\
\hline 64 & 5 & 2 & 3 & 4 & 2 & 2 & A & 14629.8246 & -0.5 \\
\hline 65 & 5 & 2 & 3 & 4 & 2 & 2 & $E$ & 14627.6673 & 1.2 \\
\hline 66 & 6 & 2 & 4 & 5 & 2 & 3 & A & 17736.8437 & 0.1 \\
\hline 67 & 6 & 2 & 4 & 5 & 2 & 3 & $E$ & 17735.1479 & 1.8 \\
\hline 68 & 7 & 3 & 5 & 6 & 3 & 4 & A & 19918.1787 & -1.6 \\
\hline 69 & 7 & 3 & 5 & 6 & 3 & 4 & $\mathrm{E}$ & 19925.1530 & 0.1 \\
\hline 70 & 5 & 3 & 3 & 4 & 3 & 2 & A & 14197.5575 & -0.9 \\
\hline 71 & 5 & 3 & 3 & 4 & 3 & 2 & $E$ & 14209.8716 & 1.2 \\
\hline 72 & 6 & 3 & 4 & 5 & 3 & 3 & A & 17058.1156 & -0.7 \\
\hline
\end{tabular}




\begin{tabular}{lllllllllr}
\hline $\mathbf{7 3}$ & 7 & 3 & 5 & 6 & 3 & 4 & $\mathrm{~A}$ & 19918.1797 & -0.6 \\
$\mathbf{7 4}$ & 5 & 3 & 2 & 4 & 3 & 1 & $\mathrm{~A}$ & 14234.4657 & -0.2 \\
$\mathbf{7 5}$ & 5 & 3 & 2 & 4 & 3 & 1 & $\mathrm{E}$ & 14220.6060 & -0.9 \\
$\mathbf{7 6}$ & 6 & 3 & 3 & 5 & 3 & 2 & $\mathrm{~A}$ & 17154.6940 & -0.5 \\
$\mathbf{7 7}$ & 6 & 3 & 3 & 5 & 3 & 2 & $\mathrm{E}$ & 17138.6121 & 0.7 \\
$\mathbf{7 8}$ & 7 & 3 & 4 & 6 & 3 & 3 & $\mathrm{~A}$ & 20128.5831 & -1.6 \\
$\mathbf{7 9}$ & 7 & 3 & 4 & 6 & 3 & 3 & $\mathrm{E}$ & 20119.2971 & 0.8 \\
$\mathbf{8 0}$ & 5 & 4 & 2 & 4 & 4 & 1 & $\mathrm{~A}$ & 14174.5898 & 0.8 \\
$\mathbf{8 1}$ & 6 & 4 & 3 & 5 & 4 & 2 & $\mathrm{~A}$ & 17033.0945 & 0.2 \\
\hline
\end{tabular}

Table S7e: Observed frequencies $v_{\text {obs }}$ of 81 rotational transitions of the ${ }^{13} \mathrm{C}(5)$ isotopologue (for atom numbering see Figure 1) of anti-2AF with $v_{o b s}-v_{\text {calc }}$ values obtained from a fit with the program $X I A M$.

\begin{tabular}{|c|c|c|c|c|c|c|c|c|c|}
\hline No. & \multicolumn{3}{|c|}{ upper level } & $\begin{array}{l}J \\
\text { low }\end{array}$ & lower level & $\frac{K_{c}}{\text { el }}$ & \multirow{2}{*}{$\frac{\text { Species }}{\mathrm{A}}$} & \multirow{2}{*}{$\begin{array}{c}\begin{array}{c}\text { Vobs } \\
\text { [MHz] }\end{array} \\
5618.5595\end{array}$} & \multirow{2}{*}{$\begin{array}{r}\begin{array}{r}\mathbf{V}_{\text {obs }}-\mathbf{V}_{\text {calc }} \\
{[\mathbf{k H z}]}\end{array} \\
-0.1\end{array}$} \\
\hline 1 & 2 & 0 & 2 & 1 & 0 & 1 & & & \\
\hline 2 & 3 & 0 & 3 & 2 & 0 & 2 & A & 8344.8439 & 0.9 \\
\hline 3 & 3 & 0 & 3 & 2 & 0 & 2 & & 8344.4620 & -0.8 \\
\hline 4 & 4 & 0 & 4 & 3 & 0 & 3 & & 56 & 1.3 \\
\hline 5 & 4 & 0 & 4 & 3 & 0 & 3 & & 27 & -0.4 \\
\hline 6 & 5 & 0 & 5 & 4 & 0 & 4 & & 31 & 0.6 \\
\hline 7 & 5 & 0 & 5 & 4 & 0 & 4 & & 93 & 0.0 \\
\hline 8 & 6 & 0 & 6 & 5 & c & 5 & & & -0.2 \\
\hline 9 & 6 & 0 & 6 & 5 & c & 5 & & & 0.9 \\
\hline 10 & 7 & 0 & 7 & 6 & c & 6 & & 69 & -0.6 \\
\hline 11 & 7 & 0 & 7 & 6 & 0 & 6 & & & 0.4 \\
\hline 12 & 8 & 0 & 8 & 7 & 0 & 7 & & & 0.2 \\
\hline 13 & 8 & 0 & 8 & 7 & 0 & 7 & & & 1.0 \\
\hline 14 & 3 & 0 & 3 & 2 & 1 & 2 & & & 2.8 \\
\hline 15 & 3 & 0 & 3 & 2 & 1 & 2 & & & -1.3 \\
\hline 16 & 5 & 0 & 5 & 4 & 1 & 4 & & & -2.1 \\
\hline 17 & 6 & 0 & 6 & 5 & 1 & 5 & & & -0.8 \\
\hline 18 & 6 & 0 & 6 & 5 & 1 & 5 & L & 92 & 0.2 \\
\hline 19 & 7 & 0 & 7 & 6 & 1 & 6 & $\Lambda$ & 48 & -0.1 \\
\hline 20 & 7 & 0 & 7 & 6 & 1 & 6 & & & 0.2 \\
\hline 21 & 8 & 0 & 8 & 7 & 1 & 7 & & 201 & -1.2 \\
\hline 22 & 8 & 0 & 8 & 7 & 1 & 7 & & 20167.3038 & -0.4 \\
\hline 23 & 9 & 0 & 9 & 8 & 4 & 8 & 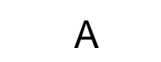 & 22789.8407 & -1.6 \\
\hline 24 & 9 & 0 & 9 & 8 & & 8 & F & 22789.4305 & 2.5 \\
\hline 25 & 4 & 1 & 4 & 3 & 0 & 3 & A & 12838.2917 & -2.1 \\
\hline 26 & 4 & 1 & 4 & 3 & 0 & 3 & $E$ & 12838.0586 & 1.9 \\
\hline
\end{tabular}




\begin{tabular}{|c|c|c|c|c|c|c|c|c|c|}
\hline 27 & 5 & 1 & 5 & 4 & 0 & 4 & A & 14876.1316 & 1.4 \\
\hline 28 & 5 & 1 & 5 & 4 & 0 & 4 & E & 14876.0333 & 3.3 \\
\hline 29 & 6 & 1 & 6 & 5 & 0 & 5 & A & 16911.5946 & 4.1 \\
\hline 30 & 6 & 1 & 6 & 5 & 0 & 5 & E & 16911.5691 & 1.3 \\
\hline 31 & 7 & 1 & 7 & 6 & 0 & 6 & A & 19000.0739 & -5.1 \\
\hline 32 & 8 & 1 & 8 & 7 & 0 & 7 & A & 21167.4893 & -0.4 \\
\hline 33 & 8 & 1 & 8 & 7 & 0 & 7 & E & 21167.4608 & 1.2 \\
\hline 34 & 9 & 1 & 9 & 8 & 0 & 8 & A & 23413.0722 & 0.4 \\
\hline 35 & 9 & 1 & 9 & 8 & 0 & 8 & E & 23413.0011 & 0.2 \\
\hline 36 & 3 & 1 & 3 & 2 & 1 & 2 & A & 7868.7492 & -3.9 \\
\hline 37 & 3 & 1 & 3 & 2 & 1 & 2 & $E$ & 7868.6532 & 0.5 \\
\hline 38 & 4 & 1 & 4 & 3 & 1 & 3 & A & 10456.2053 & -0.4 \\
\hline 39 & 4 & 1 & 4 & 3 & 1 & 3 & $E$ & 10455.9991 & -0.4 \\
\hline 40 & 5 & 1 & 5 & 4 & 1 & 4 & A & 13018.2494 & -1.2 \\
\hline 41 & 5 & 1 & 5 & 4 & 1 & 4 & $E$ & 13017.9966 & 0.2 \\
\hline 42 & 6 & 1 & 6 & 5 & 1 & 5 & A & 15553.9988 & -4.1 \\
\hline 43 & 6 & 1 & 6 & 5 & 1 & 5 & $E$ & 15553.7267 & -0.4 \\
\hline 44 & 7 & 1 & 7 & 6 & 1 & 6 & A & 18064.9668 & 0.4 \\
\hline 45 & 7 & 1 & 7 & 6 & 1 & 6 & E & 18064.6852 & 0.2 \\
\hline 46 & 8 & 1 & 8 & 7 & 1 & 7 & A & 20554.3967 & -1.4 \\
\hline 47 & 8 & 1 & 8 & 7 & 1 & 7 & $\mathrm{E}$ & 20554.1211 & 0.5 \\
\hline 48 & 3 & 1 & 2 & 2 & 1 & 1 & A & 9046.0074 & 0.4 \\
\hline 49 & 3 & 1 & 2 & 2 & 1 & 1 & $\mathrm{E}$ & 9045.2730 & 0.9 \\
\hline 50 & 4 & 1 & 3 & 3 & 1 & 2 & A & 12017.3847 & 0.3 \\
\hline 51 & 4 & 1 & 3 & 3 & 1 & 2 & $\mathrm{E}$ & 12016.5001 & 0.3 \\
\hline 52 & 5 & 1 & 4 & 4 & 1 & 3 & A & 14945.5159 & -0.7 \\
\hline 53 & 5 & 1 & 4 & 4 & 1 & 3 & $E$ & 14944.4686 & -0.1 \\
\hline 54 & 6 & 1 & 5 & 5 & 1 & 4 & A & 17813.0842 & 0.3 \\
\hline 55 & 6 & 1 & 5 & 5 & 1 & 4 & $\mathrm{E}$ & 17811.9173 & 0.7 \\
\hline 56 & 7 & 1 & 6 & 6 & 1 & 5 & A & 20600.3407 & 0.1 \\
\hline 57 & 7 & 1 & 6 & 6 & 1 & 5 & E & 20599.1278 & -0.2 \\
\hline 58 & 8 & 1 & 7 & 7 & 1 & 6 & A & 23288.7766 & 0.3 \\
\hline 59 & 8 & 1 & 7 & 7 & 1 & 6 & E & 23287.6002 & -0.9 \\
\hline 60 & 2 & 2 & 1 & 1 & 1 & 0 & A & 15697.4975 & -0.3 \\
\hline 61 & 3 & 2 & 2 & 2 & 1 & 1 & A & 18130.6708 & 2.3 \\
\hline 62 & 3 & 2 & 2 & 2 & 1 & 1 & $E$ & 18123.5283 & 0.1 \\
\hline 63 & 4 & 2 & 3 & 3 & 1 & 2 & A & 20363.0929 & 1.7 \\
\hline 64 & 4 & 2 & 3 & 3 & 1 & 2 & E & 20359.8098 & -2.7 \\
\hline 65 & 5 & 2 & 4 & 4 & 1 & 3 & A & 22401.5230 & -3.3 \\
\hline 66 & 4 & 2 & 2 & 3 & 2 & 1 & A & 11602.7437 & 0.2 \\
\hline 67 & 5 & 2 & 3 & 4 & 2 & 2 & A & 14667.6229 & 0.4 \\
\hline 68 & 6 & 2 & 4 & 5 & 2 & 3 & A & 17783.4144 & 1.2 \\
\hline 69 & 7 & 2 & 5 & 6 & 2 & 4 & A & 20910.0802 & -1.2 \\
\hline
\end{tabular}




\begin{tabular}{rrlllllllr}
\hline $\mathbf{7 0}$ & 4 & 2 & 3 & 3 & 2 & 2 & $\mathrm{~A}$ & 11278.4291 & -0.6 \\
$\mathbf{7 1}$ & 5 & 2 & 4 & 4 & 2 & 3 & $\mathrm{~A}$ & 14055.8203 & 0.8 \\
$\mathbf{7 2}$ & 6 & 2 & 5 & 5 & 2 & 4 & $\mathrm{~A}$ & 16805.6389 & 1.0 \\
$\mathbf{7 3}$ & 7 & 2 & 6 & 6 & 2 & 5 & $\mathrm{~A}$ & 19523.3867 & 0.7 \\
$\mathbf{7 4}$ & 5 & 3 & 2 & 4 & 3 & 1 & $\mathrm{~A}$ & 14267.1945 & 0.3 \\
$\mathbf{7 5}$ & 6 & 3 & 3 & 5 & 3 & 2 & $\mathrm{~A}$ & 17195.3992 & -1.1 \\
$\mathbf{7 6}$ & 5 & 3 & 3 & 4 & 3 & 2 & $\mathrm{~A}$ & 14229.3354 & 0.1 \\
$\mathbf{7 7}$ & 5 & 3 & 3 & 4 & 3 & 2 & $\mathrm{E}$ & 14241.6943 & 2.2 \\
$\mathbf{7 8}$ & 6 & 3 & 4 & 5 & 3 & 3 & $\mathrm{~A}$ & 17096.3710 & 0.2 \\
$\mathbf{7 9}$ & 6 & 3 & 4 & 5 & 3 & 3 & $\mathrm{E}$ & 17110.0254 & -1.6 \\
$\mathbf{8 0}$ & 8 & 3 & 6 & 7 & 3 & 5 & $\mathrm{~A}$ & 22820.9182 & 0.2 \\
$\mathbf{8 1}$ & 8 & 3 & 6 & 7 & 3 & 5 & $\mathrm{E}$ & 22822.7255 & 1.1 \\
\hline
\end{tabular}

Table S7f: Observed frequencies $v_{\text {obs }}$ of 81 rotational transitions of the ${ }^{13} \mathrm{C}(6)$ isotopologue (for atom numbering see Figure 1) of anti-2AF with $v_{o b s}-v_{\text {calc }}$ values obtained from a fit with the program XIAM.

\begin{tabular}{|c|c|c|c|c|c|c|c|c|c|}
\hline \multirow[t]{2}{*}{ No. } & $\boldsymbol{J}$ & $K_{a}$ & $K_{c}$ & $J$ & $K_{a}$ & $K_{c}$ & & & \\
\hline & \multicolumn{3}{|c|}{ upper level } & \multicolumn{4}{|c|}{ lower level } & [MHz] & [kHz] \\
\hline 1 & 3 & 0 & 3 & 2 & 0 & 2 & A & 8401.3200 & 0.2 \\
\hline 2 & 3 & 0 & 3 & 2 & 0 & 2 & $E$ & 8400.9369 & -0.9 \\
\hline 3 & 4 & 0 & 4 & 3 & 0 & 3 & A & 11054.0272 & 0.3 \\
\hline 4 & 4 & 0 & 4 & 3 & 0 & 3 & $\mathrm{E}$ & 11053.6333 & -0.1 \\
\hline 5 & 5 & 0 & 5 & 4 & 0 & 4 & A & 13608.3399 & 0.2 \\
\hline 6 & 5 & 0 & 5 & 4 & 0 & 4 & $\mathrm{E}$ & 13607.9827 & -1.0 \\
\hline 7 & 8 & 0 & 8 & 7 & 0 & 7 & A & 20917.5413 & 0.0 \\
\hline 8 & 8 & 0 & 8 & 7 & 0 & 7 & $\mathrm{E}$ & 20917.3101 & 0.5 \\
\hline 9 & 3 & 0 & 3 & 2 & 1 & 2 & A & 5532.3762 & 2.9 \\
\hline 10 & 3 & 0 & 3 & 2 & 1 & 2 & $E$ & 5532.3187 & -1.4 \\
\hline 11 & 4 & 0 & 4 & 3 & 1 & 3 & A & 8664.7038 & 1.8 \\
\hline 12 & 4 & 0 & 4 & 3 & 1 & 3 & $E$ & 8664.3527 & -1.8 \\
\hline 13 & 5 & 0 & 5 & 4 & 1 & 4 & A & 11746.6562 & -2.9 \\
\hline 14 & 5 & 0 & 5 & 4 & 1 & 4 & E & 11746.1596 & -2.6 \\
\hline 15 & 7 & 0 & 7 & 6 & 1 & 6 & A & 17573.3262 & 0.2 \\
\hline 16 & 7 & 0 & 7 & 6 & 1 & 6 & E & 17572.8023 & -0.7 \\
\hline 17 & 8 & 0 & 8 & 7 & 1 & 7 & A & 20305.6284 & -0.6 \\
\hline 18 & 8 & 0 & 8 & 7 & 1 & 7 & $\mathrm{E}$ & 20305.1578 & 0.2 \\
\hline 19 & 3 & 1 & 3 & 2 & 0 & 2 & A & 10790.6426 & -2.0 \\
\hline 20 & 3 & 1 & 3 & 2 & 0 & 2 & $\mathrm{E}$ & 10790.2178 & 1.1 \\
\hline 21 & 4 & 1 & 4 & 3 & 0 & 3 & A & 12915.7071 & -0.4 \\
\hline 22 & 4 & 1 & 4 & 3 & 0 & 3 & $\mathrm{E}$ & 12915.4559 & 1.0 \\
\hline 23 & 5 & 1 & 5 & 4 & 0 & 4 & A & 14967.0642 & 3.3 \\
\hline
\end{tabular}




\begin{tabular}{|c|c|c|c|c|c|c|c|c|c|}
\hline 24 & 5 & 1 & 5 & 4 & 0 & 4 & $E$ & 14966.9469 & 0.5 \\
\hline 25 & 6 & 1 & 6 & 5 & 0 & 5 & A & 17016.5349 & -3.5 \\
\hline 26 & 6 & 1 & 6 & 5 & 0 & 5 & $\mathrm{E}$ & 17016.5040 & 1.4 \\
\hline 27 & 7 & 1 & 7 & 6 & 0 & 6 & A & 19119.8707 & 1.3 \\
\hline 28 & 7 & 1 & 7 & 6 & 0 & 6 & E & 19119.8515 & -0.3 \\
\hline 29 & 8 & 1 & 8 & 7 & 0 & 7 & A & 21302.8673 & -1.9 \\
\hline 30 & 8 & 1 & 8 & 7 & 0 & 7 & E & 21302.8306 & 1.5 \\
\hline 31 & 3 & 1 & 3 & 2 & 1 & 2 & A & 7921.7000 & 1.8 \\
\hline 32 & 3 & 1 & 3 & 2 & 1 & 2 & $E$ & 7921.5979 & -1.1 \\
\hline 33 & 4 & 1 & 4 & 3 & 1 & 3 & A & 10526.3824 & -0.3 \\
\hline 34 & 4 & 1 & 4 & 3 & 1 & 3 & $\mathrm{E}$ & 10526.1760 & -0.1 \\
\hline 35 & 5 & 1 & 5 & 4 & 1 & 4 & A & 13105.3797 & -0.6 \\
\hline 36 & 5 & 1 & 5 & 4 & 1 & 4 & $E$ & 13105.1237 & -1.2 \\
\hline 37 & 6 & 1 & 6 & 5 & 1 & 5 & A & 15657.8174 & 0.2 \\
\hline 38 & 6 & 1 & 6 & 5 & 1 & 5 & $E$ & 15657.5406 & 0.8 \\
\hline 39 & 8 & 1 & 8 & 7 & 1 & 7 & A & 20690.9567 & -0.2 \\
\hline 40 & 8 & 1 & 8 & 7 & 1 & 7 & $\mathrm{E}$ & 20690.6777 & 0.6 \\
\hline 41 & 3 & 1 & 2 & 2 & 1 & 1 & A & 9108.7258 & 0.5 \\
\hline 42 & 3 & 1 & 2 & 2 & 1 & 1 & $\mathrm{E}$ & 9107.9859 & 1.0 \\
\hline 43 & 4 & 1 & 3 & 3 & 1 & 2 & A & 12100.4576 & 1.0 \\
\hline 44 & 4 & 1 & 3 & 3 & 1 & 2 & $E$ & 12099.5669 & 0.1 \\
\hline 45 & 5 & 1 & 4 & 4 & 1 & 3 & A & 15048.3816 & 0.5 \\
\hline 46 & 5 & 1 & 4 & 4 & 1 & 3 & $E$ & 15047.3269 & -0.8 \\
\hline 47 & 6 & 1 & 5 & 5 & 1 & 4 & A & 17934.9419 & 0.2 \\
\hline 48 & 6 & 1 & 5 & 5 & 1 & 4 & E & 17933.7674 & -1.1 \\
\hline 49 & 7 & 1 & 6 & 6 & 1 & 5 & A & 20740.1425 & 0.9 \\
\hline 50 & 7 & 1 & 6 & 6 & 1 & 5 & E & 20738.9218 & -0.9 \\
\hline 51 & 8 & 1 & 7 & 7 & 1 & 6 & A & 23445.3026 & 0.0 \\
\hline 52 & 8 & 1 & 7 & 7 & 1 & 6 & $E$ & 23444.1232 & 2.6 \\
\hline 53 & 2 & 2 & 1 & 1 & 1 & 0 & A & 15780.3675 & 1.8 \\
\hline 54 & 3 & 2 & 2 & 2 & 1 & 1 & A & 18229.6342 & 1.8 \\
\hline 55 & 3 & 2 & 2 & 2 & 1 & 1 & $\mathrm{E}$ & 18222.3467 & -3.0 \\
\hline 56 & 4 & 2 & 3 & 3 & 1 & 2 & A & 20476.4673 & -2.4 \\
\hline 57 & 4 & 2 & 2 & 3 & 2 & 1 & A & 11683.7160 & 0.3 \\
\hline 58 & 4 & 2 & 2 & 3 & 2 & 1 & $E$ & 11679.2230 & 0.6 \\
\hline 59 & 5 & 2 & 3 & 4 & 2 & 2 & A & 14770.5321 & -0.6 \\
\hline 60 & 5 & 2 & 3 & 4 & 2 & 2 & $\mathrm{E}$ & 14768.4060 & 2.1 \\
\hline 61 & 6 & 2 & 4 & 5 & 2 & 3 & A & 17908.4497 & -0.1 \\
\hline 62 & 6 & 2 & 4 & 5 & 2 & 3 & $E$ & 17906.7389 & 0.8 \\
\hline 63 & 7 & 2 & 5 & 6 & 2 & 4 & A & 21056.8707 & -0.9 \\
\hline 64 & 7 & 2 & 5 & 6 & 2 & 4 & E & 21055.1280 & 0.6 \\
\hline 65 & 5 & 2 & 4 & 4 & 2 & 3 & A & 14151.7222 & -0.8 \\
\hline 66 & 5 & 2 & 4 & 4 & 2 & 3 & $E$ & 14152.1608 & 0.9 \\
\hline
\end{tabular}




\begin{tabular}{lllllllllr}
\hline $\mathbf{6 7}$ & 6 & 2 & 5 & 5 & 2 & 4 & $\mathrm{~A}$ & 16919.9837 & 1.7 \\
$\mathbf{6 8}$ & 6 & 2 & 5 & 5 & 2 & 4 & $\mathrm{E}$ & 16919.6260 & 1.1 \\
$\mathbf{6 9}$ & 7 & 2 & 6 & 6 & 2 & 5 & $\mathrm{~A}$ & 19655.7944 & 0.4 \\
$\mathbf{7 0}$ & 7 & 2 & 6 & 6 & 2 & 5 & $\mathrm{E}$ & 19655.1250 & -0.8 \\
$\mathbf{7 1}$ & 5 & 3 & 2 & 4 & 3 & 1 & $\mathrm{~A}$ & 14365.7450 & 0.0 \\
$\mathbf{7 2}$ & 7 & 3 & 4 & 6 & 3 & 3 & $\mathrm{~A}$ & 20319.0416 & 0.7 \\
$\mathbf{7 3}$ & 5 & 3 & 3 & 4 & 3 & 2 & $\mathrm{~A}$ & 14327.2817 & -1.5 \\
$\mathbf{7 4}$ & 5 & 3 & 3 & 4 & 3 & 2 & $\mathrm{E}$ & 14339.8422 & 3.3 \\
$\mathbf{7 5}$ & 6 & 3 & 4 & 5 & 3 & 3 & $\mathrm{~A}$ & 17214.0787 & -1.3 \\
$\mathbf{7 6}$ & 6 & 3 & 4 & 5 & 3 & 3 & $\mathrm{E}$ & 17227.9392 & -0.3 \\
$\mathbf{7 7}$ & 7 & 3 & 5 & 6 & 3 & 4 & $\mathrm{~A}$ & 20100.1021 & -1.2 \\
$\mathbf{7 8}$ & 7 & 3 & 5 & 6 & 3 & 4 & $\mathrm{E}$ & 20106.6295 & -2.9 \\
$\mathbf{7 9}$ & 5 & 4 & 2 & 4 & 4 & 1 & $\mathrm{~A}$ & 14303.8897 & -1.3 \\
$\mathbf{8 0}$ & 5 & 4 & 2 & 4 & 4 & 1 & $\mathrm{E}$ & 14303.5760 & 4.1 \\
$\mathbf{8 1}$ & 6 & 4 & 3 & 5 & 4 & 2 & $\mathrm{~A}$ & 17188.9019 & -1.5 \\
\hline
\end{tabular}

Table S7g: Observed frequencies $v_{\text {obs }}$ of 40 rotational transitions of the ${ }^{18} \mathrm{O}(7)$ isotopologue (for atom numbering see Figure 1) of anti-2AF with $v_{o b s}-v_{\text {calc }}$ values obtained from a fit with the program XIAM.

\begin{tabular}{|c|c|c|c|c|c|c|c|c|c|}
\hline No. & \multicolumn{3}{|c|}{ upper level } & \multicolumn{4}{|c|}{ lower level } & $\begin{array}{c}\text { Vobs } \\
{[\mathrm{MHz}]}\end{array}$ & $\begin{array}{c}\mathbf{V}_{\text {obs }}-\mathbf{V}_{\text {calc }} \\
{[\mathrm{kHz}]}\end{array}$ \\
\hline 1 & 2 & 0 & 2 & 1 & 0 & 1 & A & 5541.3108 & -0.7 \\
\hline 2 & 2 & 0 & 2 & 1 & 0 & 1 & $\mathrm{E}$ & 5541.0093 & -1.2 \\
\hline 3 & 3 & 0 & 3 & 2 & 0 & 2 & A & 8228.4674 & 0.1 \\
\hline 4 & 3 & 0 & 3 & 2 & 0 & 2 & E & 8228.0921 & -0.5 \\
\hline 5 & 4 & 0 & 4 & 3 & 0 & 3 & A & 10824.5971 & -0.7 \\
\hline 6 & 4 & 0 & 4 & 3 & 0 & 3 & $\mathrm{E}$ & 10824.2152 & -0.5 \\
\hline 7 & 5 & 0 & 5 & 4 & 0 & 4 & A & 13323.5124 & 0.8 \\
\hline 8 & 5 & 0 & 5 & 4 & 0 & 4 & $\mathrm{E}$ & 13323.1706 & 0.1 \\
\hline 9 & 6 & 0 & 6 & 5 & 0 & 5 & A & 15743.2734 & 0.6 \\
\hline 10 & 6 & 0 & 6 & 5 & 0 & 5 & $\mathrm{E}$ & 15742.9871 & -1.0 \\
\hline 11 & 7 & 0 & 7 & 6 & 0 & 6 & A & 18117.0899 & 3.0 \\
\hline 12 & 7 & 0 & 7 & 6 & 0 & 6 & E & 18116.8458 & -1.0 \\
\hline 13 & 6 & 0 & 6 & 5 & 1 & 5 & A & 14432.9859 & -1.6 \\
\hline 14 & 6 & 0 & 6 & 5 & 1 & 5 & $\mathrm{E}$ & 14432.4223 & -2.4 \\
\hline 15 & 2 & 1 & 2 & 1 & 1 & 1 & A & .3272 & -3.0 \\
\hline 16 & 2 & 1 & 2 & 1 & 1 & 1 & $E$ & & 2.4 \\
\hline 17 & 4 & 1 & 4 & 3 & 1 & 3 & A & 10307.7571 & 0.6 \\
\hline 18 & 4 & 1 & 4 & 3 & 1 & 3 & E & 10307.5481 & -1.3 \\
\hline 19 & 5 & 1 & 5 & 4 & 1 & 4 & A & 12832.4969 & 0.4 \\
\hline 20 & 5 & 1 & 5 & 4 & 1 & 4 & $E$ & 12832.2439 & -0.8 \\
\hline
\end{tabular}




\begin{tabular}{llllllllrr}
\hline $\mathbf{2 1}$ & 6 & 1 & 6 & 5 & 1 & 5 & $\mathrm{~A}$ & 15330.9610 & -1.8 \\
$\mathbf{2 2}$ & 6 & 1 & 6 & 5 & 1 & 5 & $\mathrm{E}$ & 15330.6911 & -0.5 \\
$\mathbf{2 3}$ & 7 & 1 & 7 & 6 & 1 & 6 & $\mathrm{~A}$ & 17804.7523 & -0.6 \\
$\mathbf{2 4}$ & 7 & 1 & 7 & 6 & 1 & 6 & $\mathrm{E}$ & 17804.4769 & -0.7 \\
$\mathbf{2 5}$ & 3 & 1 & 2 & 2 & 1 & 1 & $\mathrm{~A}$ & 8925.8516 & -0.4 \\
$\mathbf{2 6}$ & 3 & 1 & 2 & 2 & 1 & 1 & $\mathrm{E}$ & 8925.1323 & -0.4 \\
$\mathbf{2 7}$ & 4 & 1 & 3 & 3 & 1 & 2 & $\mathrm{~A}$ & 11856.8199 & 0.4 \\
$\mathbf{2 8}$ & 4 & 1 & 3 & 3 & 1 & 2 & $\mathrm{E}$ & 11855.9487 & 2.2 \\
$\mathbf{2 9}$ & 5 & 1 & 4 & 4 & 1 & 3 & $\mathrm{~A}$ & 14744.1214 & 0.5 \\
$\mathbf{3 0}$ & 5 & 1 & 4 & 4 & 1 & 3 & $\mathrm{E}$ & 14743.0868 & 0.9 \\
$\mathbf{3 1}$ & 6 & 1 & 5 & 5 & 1 & 4 & $\mathrm{~A}$ & 17570.2231 & 1.1 \\
$\mathbf{3 2}$ & 6 & 1 & 5 & 5 & 1 & 4 & $\mathrm{E}$ & 17569.0736 & 1.6 \\
$\mathbf{3 3}$ & 3 & 1 & 3 & 2 & 0 & 2 & $\mathrm{~A}$ & 10546.6068 & -2.1 \\
$\mathbf{3 4}$ & 3 & 1 & 3 & 2 & 0 & 2 & $\mathrm{E}$ & 10546.2482 & 0.1 \\
$\mathbf{3 5}$ & 4 & 1 & 4 & 3 & 0 & 3 & $\mathrm{~A}$ & 12625.9022 & 4.0 \\
$\mathbf{3 6}$ & 4 & 1 & 4 & 3 & 0 & 3 & $\mathrm{E}$ & 12625.7062 & 1.3 \\
$\mathbf{3 7}$ & 5 & 1 & 5 & 4 & 0 & 4 & $\mathrm{~A}$ & 14633.7980 & 1.1 \\
$\mathbf{3 8}$ & 5 & 1 & 5 & 4 & 0 & 4 & $\mathrm{E}$ & 14633.7335 & -0.4 \\
$\mathbf{3 9}$ & 6 & 1 & 6 & 5 & 0 & 5 & $\mathrm{E}$ & 16641.2550 & 0.0 \\
$\mathbf{4 0}$ & 2 & 2 & 1 & 1 & 1 & 0 & $\mathrm{~A}$ & 15400.5522 & -2.2 \\
\hline
\end{tabular}

Table S7h: Observed frequencies $v_{\text {obs }}$ of 82 rotational transitions of the ${ }^{13} \mathrm{C}(8)$ isotopologue (for atom numbering see Figure 1) of anti-2AF with $v_{o b s}-v_{\text {calc }}$ values obtained from a fit with the program XIAM.

\begin{tabular}{|c|c|c|c|c|c|c|c|c|c|}
\hline \multirow[t]{2}{*}{ No. } & $J$ & $K_{a}$ & $K_{c}$ & $J$ & $K_{a}$ & $K_{c}$ & Species & $V_{\text {obs }}$ & $V_{\text {obs }}-V_{\text {calc }}$ \\
\hline & \multicolumn{3}{|c|}{ upper level } & \multicolumn{3}{|c|}{ lower level } & & [MHz] & \\
\hline 1 & 2 & 0 & 2 & 1 & 0 & 1 & A & 5608.9398 & 0.4 \\
\hline 2 & 2 & 0 & 2 & 1 & 0 & 1 & $E$ & 5608.6422 & -1.7 \\
\hline 3 & 3 & 0 & 3 & 2 & 0 & 2 & A & 8328.5902 & -0.3 \\
\hline 4 & 3 & 0 & 3 & 2 & 0 & 2 & E & 8328.2200 & -0.8 \\
\hline 5 & 4 & 0 & 4 & 3 & 0 & 3 & A & 10955.8258 & 0.0 \\
\hline 6 & 4 & 0 & 4 & 3 & 0 & 3 & $E$ & 10955.4448 & -0.4 \\
\hline 7 & 5 & 0 & 5 & 4 & 0 & 4 & A & 13484.4691 & -0.5 \\
\hline 8 & 5 & 0 & 5 & 4 & 0 & 4 & $E$ & 13484.1256 & 0.7 \\
\hline 9 & 6 & 0 & 6 & 5 & 0 & 5 & A & 15933.0079 & -0.5 \\
\hline 10 & 6 & 0 & 6 & 5 & 0 & 5 & E & 15932.7161 & 0.9 \\
\hline 11 & 7 & 0 & 7 & 6 & 0 & 6 & A & 18335.2146 & -0.8 \\
\hline 12 & 7 & 0 & 7 & 6 & 0 & 6 & $E$ & 18334.9656 & 1.6 \\
\hline 13 & 8 & 0 & 8 & 7 & 0 & 7 & A & 20722.0355 & 7.8 \\
\hline 14 & 8 & 0 & 8 & 7 & 0 & 7 & $E$ & 20721.7933 & -6.9 \\
\hline 15 & 3 & 0 & 3 & 2 & 1 & 2 & A & 5512.0164 & 2.7 \\
\hline
\end{tabular}




\begin{tabular}{|c|c|c|c|c|c|c|c|c|c|}
\hline 16 & 3 & 0 & 3 & 2 & 1 & 2 & $E$ & 5511.9967 & -0.5 \\
\hline 17 & 4 & 0 & 4 & 3 & 1 & 3 & A & 8616.0457 & -0.7 \\
\hline 18 & 4 & 0 & 4 & 3 & 1 & 3 & E & 8615.7384 & -0.6 \\
\hline 19 & 5 & 0 & 5 & 4 & 1 & 4 & A & 11667.6582 & -1.6 \\
\hline 20 & 5 & 0 & 5 & 4 & 1 & 4 & $E$ & 11667.2046 & -0.3 \\
\hline 21 & 6 & 0 & 6 & 5 & 1 & 5 & A & 14612.5978 & -0.1 \\
\hline 22 & 6 & 0 & 6 & 5 & 1 & 5 & $E$ & 14612.0957 & 0.4 \\
\hline 23 & 7 & 0 & 7 & 6 & 1 & 6 & A & 17431.1828 & -0.1 \\
\hline 24 & 7 & 0 & 7 & 6 & 1 & 6 & $E$ & 17430.6970 & 0.8 \\
\hline 25 & 8 & 0 & 8 & 7 & 1 & 7 & A & 20133.0349 & -0.4 \\
\hline 26 & 8 & 0 & 8 & 7 & 1 & 7 & $E$ & 20132.5960 & 1.5 \\
\hline 27 & 3 & 1 & 3 & 2 & 0 & 2 & A & 10668.3642 & -5.8 \\
\hline 28 & 3 & 1 & 3 & 2 & 0 & 2 & $E$ & 10667.9268 & -0.2 \\
\hline 29 & 4 & 1 & 4 & 3 & 0 & 3 & A & 12772.6317 & -4.0 \\
\hline 30 & 4 & 1 & 4 & 3 & 0 & 3 & $E$ & 12772.3660 & 0.9 \\
\hline 31 & 5 & 1 & 5 & 4 & 0 & 4 & A & 14804.8820 & 1.8 \\
\hline 32 & 5 & 1 & 5 & 4 & 0 & 4 & $E$ & 14804.7458 & 1.0 \\
\hline 33 & 6 & 1 & 6 & 5 & 0 & 5 & A & 16837.0408 & -0.1 \\
\hline 34 & 6 & 1 & 6 & 5 & 0 & 5 & $E$ & 16836.9842 & 1.2 \\
\hline 35 & 7 & 1 & 7 & 6 & 0 & 6 & A & 18924.2058 & -2.0 \\
\hline 36 & 7 & 1 & 7 & 6 & 0 & 6 & $E$ & 18924.1716 & 1.9 \\
\hline 37 & 8 & 1 & 8 & 7 & 0 & 7 & A & 21091.0996 & -3.3 \\
\hline 38 & 8 & 1 & 8 & 7 & 0 & 7 & $E$ & 21091.0480 & 1.8 \\
\hline 39 & 3 & 1 & 2 & 2 & 1 & 1 & A & 9035.4394 & 1.0 \\
\hline 40 & 3 & 1 & 2 & 2 & 1 & 1 & $\mathrm{E}$ & 9034.7174 & 1.9 \\
\hline 41 & 4 & 1 & 3 & 3 & 1 & 2 & A & 12002.2176 & 0.6 \\
\hline 42 & 4 & 1 & 3 & 3 & 1 & 2 & $\mathrm{E}$ & 12001.3522 & -0.2 \\
\hline 43 & 5 & 1 & 4 & 4 & 1 & 3 & A & 14924.6086 & -0.2 \\
\hline 44 & 5 & 1 & 4 & 4 & 1 & 3 & $E$ & 14923.5863 & -0.5 \\
\hline 45 & 6 & 1 & 5 & 5 & 1 & 4 & A & 17784.7832 & 1.7 \\
\hline 46 & 6 & 1 & 5 & 5 & 1 & 4 & E & 17783.6447 & 0.0 \\
\hline 47 & 7 & 1 & 6 & 6 & 1 & 5 & A & 20562.4959 & -0.3 \\
\hline 48 & 8 & 1 & 7 & 7 & 1 & 6 & A & 23239.0920 & 0.5 \\
\hline 49 & 8 & 1 & 7 & 7 & 1 & 6 & $E$ & 23237.9472 & -1.3 \\
\hline 50 & 3 & 1 & 3 & 2 & 1 & 2 & A & 7851.7932 & 0.0 \\
\hline 51 & 3 & 1 & 3 & 2 & 1 & 2 & $E$ & 7851.7030 & -0.4 \\
\hline 52 & 4 & 1 & 4 & 3 & 1 & 3 & A & 10432.8557 & -0.5 \\
\hline 53 & 4 & 1 & 4 & 3 & 1 & 3 & E & 10432.6587 & -0.3 \\
\hline 54 & 5 & 1 & 5 & 4 & 1 & 4 & A & 12988.0694 & -1.0 \\
\hline 55 & 5 & 1 & 5 & 4 & 1 & 4 & $E$ & 12987.8252 & 0.4 \\
\hline 56 & 6 & 1 & 6 & 5 & 1 & 5 & A & 15516.6298 & -0.6 \\
\hline 57 & 6 & 1 & 6 & 5 & 1 & 5 & $E$ & 15516.3631 & 0.0 \\
\hline 58 & 8 & 1 & 8 & 7 & 1 & 7 & A & 20502.1097 & -0.8 \\
\hline
\end{tabular}




\begin{tabular}{lllllllllr}
\hline $\mathbf{5 9}$ & 2 & 2 & 1 & 1 & 1 & 0 & $\mathrm{~A}$ & 15570.1594 & -2.3 \\
$\mathbf{6 0}$ & 2 & 2 & 1 & 1 & 1 & 0 & $\mathrm{E}$ & 15548.9478 & 2.9 \\
$\mathbf{6 1}$ & 3 & 2 & 2 & 2 & 1 & 1 & $\mathrm{~A}$ & 17996.7457 & -5.0 \\
$\mathbf{6 2}$ & 3 & 2 & 2 & 2 & 1 & 1 & $\mathrm{E}$ & 17989.3687 & -4.2 \\
$\mathbf{6 3}$ & 4 & 2 & 3 & 3 & 1 & 2 & $\mathrm{~A}$ & 20221.4421 & 6.2 \\
$\mathbf{6 4}$ & 5 & 2 & 4 & 4 & 1 & 3 & $\mathrm{~A}$ & 22251.2089 & 4.7 \\
$\mathbf{6 5}$ & 4 & 2 & 2 & 3 & 2 & 1 & $\mathrm{~A}$ & 11591.3123 & 0.2 \\
$\mathbf{6 6}$ & 5 & 2 & 3 & 4 & 2 & 2 & $\mathrm{~A}$ & 14655.6870 & 0.7 \\
$\mathbf{6 7}$ & 6 & 2 & 4 & 5 & 2 & 3 & $\mathrm{~A}$ & 17770.1849 & 0.5 \\
$\mathbf{6 8}$ & 7 & 2 & 5 & 6 & 2 & 4 & $\mathrm{~A}$ & 20893.5477 & 2.0 \\
$\mathbf{6 9}$ & 5 & 2 & 4 & 4 & 2 & 3 & $\mathrm{~A}$ & 14031.9849 & -0.3 \\
$\mathbf{7 0}$ & 5 & 2 & 4 & 4 & 2 & 3 & $\mathrm{E}$ & 14032.4587 & 2.4 \\
$\mathbf{7 1}$ & 6 & 2 & 5 & 5 & 2 & 4 & $\mathrm{~A}$ & 16775.6738 & -0.3 \\
$\mathbf{7 2}$ & 6 & 2 & 5 & 5 & 2 & 4 & $\mathrm{E}$ & 16775.3468 & 0.1 \\
$\mathbf{7 3}$ & 5 & 3 & 2 & 4 & 3 & 1 & $\mathrm{~A}$ & 14248.4945 & -0.7 \\
$\mathbf{7 4}$ & 5 & 3 & 2 & 4 & 3 & 1 & $\mathrm{E}$ & 14234.1305 & -2.2 \\
$\mathbf{7 5}$ & 6 & 3 & 3 & 5 & 3 & 2 & $\mathrm{~A}$ & 17175.1082 & -0.9 \\
$\mathbf{7 6}$ & 6 & 3 & 3 & 5 & 3 & 2 & $\mathrm{E}$ & 17159.1864 & 0.5 \\
$\mathbf{7 7}$ & 5 & 3 & 3 & 4 & 3 & 2 & $\mathrm{~A}$ & 14209.1329 & -1.5 \\
$\mathbf{7 8}$ & 5 & 3 & 3 & 4 & 3 & 2 & $\mathrm{E}$ & 14221.9623 & 3.5 \\
$\mathbf{7 9}$ & 6 & 3 & 4 & 5 & 3 & 3 & $\mathrm{~A}$ & 17072.2206 & -2.4 \\
$\mathbf{8 0}$ & 6 & 3 & 4 & 5 & 3 & 3 & $\mathrm{E}$ & 17086.2413 & 0.5 \\
$\mathbf{8 1}$ & 8 & 3 & 6 & 7 & 3 & 5 & $\mathrm{~A}$ & 22787.5784 & -2.3 \\
$\mathbf{8 2}$ & 8 & 3 & 6 & 7 & 3 & 5 & $\mathrm{E}$ & 22789.4576 & 0.0 \\
\hline
\end{tabular}


Table S8a: Molecular parameters used for the SFLAMS fits of the A species of all assigned isotopologues of anti-2AF.

\begin{tabular}{|c|c|c|c|c|c|c|}
\hline Par. $^{a}$ & Unit & ${ }^{12} \mathrm{C}$ & ${ }^{18} \mathrm{O}(1)$ & ${ }^{13} \mathrm{C}(2)$ & ${ }^{13} \mathrm{C}(3)$ & ${ }^{13} \mathrm{C}(4)$ \\
\hline$A$ & $\mathrm{MHz}$ & $4852.41457(23)$ & 4753.9836(14) & $4851.56083(57)$ & 4788.52039(39) & $4836.3764(17)$ \\
\hline$B$ & $\mathrm{MHz}$ & $1631.332542(91)$ & $1625.94966(36)$ & $1631.41692(55)$ & $1626.71424(17)$ & $1605.37265(24)$ \\
\hline C & $\mathrm{MHz}$ & $1230.594525(80)$ & $1221.13733(26)$ & $1.23058954(31)$ & $1223.83430(12)$ & $1214.75723(15)$ \\
\hline$D_{\jmath}$ & $\mathrm{kHz}$ & $0.06708(66)$ & 0.0693(19) & - & $0.0673(11)$ & $0.0664(13)$ \\
\hline$D_{J K}$ & $\mathrm{kHz}$ & $0.1347(28)$ & - & $0.146(14)$ & $0.116(14)$ & $0.1242(79)$ \\
\hline$D_{K}$ & $\mathrm{kHz}$ & $0.556(12)$ & - & $0.641(67)$ & - & - \\
\hline$d_{1}$ & $\mathrm{MHz}$ & $-0.02090(32)$ & $-0.0197(21)$ & $-0.0322(61)$ & $-0.01724(78)$ & $-0.01861(96)$ \\
\hline$d_{2}$ & $\mathrm{kHz}$ & $-0.00312(16)$ & - & - & - & - \\
\hline$N^{o}$ & & 141 & 22 & 13 & 42 & 43 \\
\hline$\sigma^{c}$ & $\mathrm{kHz}$ & 3.0 & 1.9 & 1.7 & 2.1 & 2.0 \\
\hline Par. $^{a}$ & Unit & ${ }^{13} \mathrm{C}(5)$ & ${ }^{13} \mathrm{C}(6)$ & ${ }^{18} \mathrm{O}(7)$ & ${ }^{13} \mathrm{C}(8)$ & \\
\hline$A$ & $\mathrm{MHz}$ & $4826.97136(39)$ & $4851.91144(31)$ & $4733.91205(51)$ & $4785.62271(54)$ & \\
\hline$B$ & $\mathrm{MHz}$ & $1609.61811(17)$ & $1620.93355(14)$ & $1588.87584(27)$ & $1608.48166(21)$ & \\
\hline$C$ & $\mathrm{MHz}$ & $1216.58876(11)$ & $1224.636910(99)$ & $1198.81877(14)$ & $1213.29790(16)$ & \\
\hline$D_{J}$ & $\mathrm{kHz}$ & 0.06599(89) & 0.06738(73) & $0.0628(24)$ & $0.0654(12)$ & \\
\hline$D_{J K}$ & $\mathrm{kHz}$ & $0.1216(96)$ & $0.1262(56)$ & - & $0.099(13)$ & \\
\hline$D_{K}$ & $\mathrm{kHz}$ & - & - & - & - & \\
\hline$d_{1}$ & $\mathrm{MHz}$ & $-0.01912(86)$ & $-0.01814(71)$ & $-0.0157(17)$ & $-0.0167(10)$ & \\
\hline$d_{2}$ & $\mathrm{kHz}$ & $-0.00213(70)$ & $-0.00185(53)$ & - & - & \\
\hline$N^{\mathrm{o}}$ & & 48 & 43 & 20 & 45 & \\
\hline$\sigma^{c}$ & $\mathrm{kHz}$ & 1.8 & 1.4 & 1.5 & 2.5 & \\
\hline
\end{tabular}

a All parameters refer to the principal axis system. Watson's S reduction in the Ir representation was used.

${ }^{b}$ Number of lines.

c Standard deviation of the fit. 
Table S8b: Molecular parameters used for the SFLAMS fits of the E species of all assigned isotopologues of anti-2AF.

\begin{tabular}{|c|c|c|c|c|c|c|}
\hline Par. $^{a}$ & Unit & ${ }^{12} \mathrm{C}$ & ${ }^{18} \mathrm{O}(1)$ & ${ }^{13} \mathrm{C}(2)$ & ${ }^{13} \mathrm{C}(3)$ & ${ }^{13} \mathrm{C}(4)$ \\
\hline$A$ & $\mathrm{MHz}$ & $4851.96489(11)$ & $4753.5669(35)$ & $4851.10469(87)$ & $4788.0780(11)$ & $4835.9213(30)$ \\
\hline$B$ & $\mathrm{MHz}$ & $1631.182305(43)$ & $1625.79848(68)$ & $1631.27593(65)$ & $1626.56601(16)$ & $1605.22773(28)$ \\
\hline$C$ & $\mathrm{MHz}$ & $1230.597772(38)$ & $1221.14089(40)$ & $1230.58438(89)$ & $1223.837046(96)$ & $1214.76020(19)$ \\
\hline$D_{\jmath}$ & $\mathrm{kHz}$ & $0.06697(32)$ & $0.0636(41)$ & - & $0.06726(75)$ & $0.0677(17)$ \\
\hline$D_{J K}$ & $\mathrm{kHz}$ & $0.0949(14)$ & - & $0.244(35)$ & $0.100(17)$ & - \\
\hline$D_{K}$ & $\mathrm{kHz}$ & $0.5885(78)$ & - & - & - & - \\
\hline$d_{1}$ & $\mathrm{MHz}$ & $-0.01926(17)$ & $-0.0079(69)$ & $-0.102(16)$ & $-0.01854(64)$ & $-0.0190(12)$ \\
\hline$d_{2}$ & $\mathrm{kHz}$ & $-0.002532(97)$ & $0.0210(82)$ & $0.0218(68)$ & - & - \\
\hline$q$ & $\mathrm{MHz}$ & $15.99805(26)$ & $15.43(10)$ & $15.9554(25)$ & $15.9375(17)$ & $16.1128(16)$ \\
\hline$q_{J}$ & $\mathrm{kHz}$ & $-1.4905(62)$ & - & $-1.54(18)$ & $-1.538(48)$ & $1.477(67)$ \\
\hline$q_{k}$ & $\mathrm{kHz}$ & $-1.367(27)$ & - & - & - & - \\
\hline$r$ & $\mathrm{MHz}$ & $9.565(11)$ & $9.51(10)$ & - & $9.355(46)$ & $9.11(11)$ \\
\hline$N^{o}$ & & 152 & 21 & 13 & 38 & 38 \\
\hline$\sigma^{c}$ & $\mathrm{kHz}$ & 1.4 & 1.5 & 4.7 & 1.3 & 2.3 \\
\hline Par. $^{a}$ & Unit & ${ }^{13} \mathrm{C}(5)$ & ${ }^{13} \mathrm{C}(6)$ & ${ }^{18} \mathrm{O}(7)$ & ${ }^{13} \mathrm{C}(8)$ & \\
\hline$A$ & $\mathrm{MHz}$ & $4826.53924(39)$ & $4851.46501(51)$ & $4733.5253(11)$ & $4785.16351(82)$ & \\
\hline$B$ & $\mathrm{MHz}$ & $1609.47067(12)$ & $1620.78555(15)$ & $1588.73006(26)$ & $1608.33764(31)$ & \\
\hline$C$ & $\mathrm{MHz}$ & $1216.591951(53)$ & $1224.639825(83)$ & $1198.82155(17)$ & $1213.30129(14)$ & \\
\hline$D_{J}$ & $\mathrm{kHz}$ & $0.06308(51)$ & $0.06498(60)$ & $0.0611(18)$ & $0.0659(14)$ & \\
\hline$D_{J K}$ & $\mathrm{kHz}$ & $0.1091(91)$ & $0.142(11)$ & - & - & \\
\hline$D_{K}$ & $\mathrm{kHz}$ & - & - & - & - & \\
\hline$d_{1}$ & $\mathrm{MHz}$ & $-0.01623(35)$ & $-0.01824(66)$ & $-0.0116(39)$ & $-0.0147(13)$ & \\
\hline$d_{2}$ & $\mathrm{kHz}$ & - & $-0.00365(54)$ & $0.0122(70)$ & - & \\
\hline$q$ & $\mathrm{MHz}$ & $15.6844(12)$ & $15.9191(14)$ & $14.908(12)$ & $16.0839(14)$ & \\
\hline$q_{J}$ & $\mathrm{kHz}$ & $-1.480(33)$ & $-1.646(34)$ & - & $-1.487(75)$ & \\
\hline$q_{k}$ & $\mathrm{kHz}$ & - & - & - & - & \\
\hline$r$ & $\mathrm{MHz}$ & $9.599(28)$ & $9.631(30)$ & $9.348(46)$ & $9.216(67)$ & \\
\hline$N^{b}$ & & 33 & 38 & 20 & 37 & \\
\hline$\sigma^{c}$ & $\mathrm{kHz}$ & 0.8 & 1.1 & 0.6 & 2.5 & \\
\hline
\end{tabular}

a All parameters refer to the principal axis system. Watson's S reduction in the I representation was used.

${ }^{b}$ Number of lines.

c Standard deviation of the fit. 
Table S9a: Equilibrium rotational constants $\boldsymbol{B}_{\mathrm{e}}{ }^{\text {calc. }}$ and vibrational ground state rotational constants $\boldsymbol{B}_{0}$ calc. of anti-2AF obtained with rovibrational corrections at the MP2/6-311++G(d,p) level of theory. The differences $\boldsymbol{B}_{\mathrm{e}}{ }^{\text {calc. }}-\boldsymbol{B}_{0}{ }^{\text {calc. }}$ were used to correct the "unperturbed“ experimental constants $\boldsymbol{B}_{0}{ }^{A} \_$exp. deduced from the SFLAMS A species fits. The semi-experimental equilibrium rotational constants $\boldsymbol{B}_{\mathrm{e}}{ }^{\mathrm{SE}}=$ $\boldsymbol{B}_{0}{ }^{\text {exp. }}+\boldsymbol{B}_{\mathrm{e}}{ }^{\text {calc. }}-\boldsymbol{B}_{0}{ }^{\text {calc. }}$ were subsequently input in the program KRA to determine the $\boldsymbol{r}_{\boldsymbol{s} \rightarrow e^{s E}}$.structure. All values are given in $\mathrm{MHz}$.

\begin{tabular}{|c|c|c|c|c|c|c|c|c|c|c|}
\hline & ${ }^{12} \mathrm{C}$ & \multicolumn{9}{|c|}{${ }^{18} \mathrm{O}(1)$} \\
\hline & $B_{\mathrm{e}}^{\text {calc. }}$ & $B_{0}$ calc. & $B_{\mathrm{e}}$ calc. $-B_{0}$ calc. & $B_{0}{ }^{A} \_$exp. & $B_{\mathrm{e}} \mathrm{SE}$ & $B_{\mathrm{e}^{\text {calc. }}}$ & $B_{0}$ calc. & $B_{\mathrm{e}}^{\text {calc. }}-B_{0}^{\text {calc. }}$ & $B_{0}{ }^{A} \_$exp. & $B_{\mathrm{e}}{ }^{\mathrm{SE}}$ \\
\hline $\bar{A}$ & 4820.557 & 4790.687 & 29.870 & 4852.093 & 4881.963 & 4724.183 & 4694.620 & 29.563 & 4753.686 & 4783.249 \\
\hline$B$ & 1630.489 & 1618.187 & 12.302 & 1631.221 & 1643.523 & 1625.142 & 1612.979 & 12.163 & 1625.837 & 1638.000 \\
\hline$C$ & 1227.656 & 1219.752 & 7.904 & 1230.595 & 1238.499 & 1218.308 & 1210.508 & 7.800 & 1221.137 & 1228.937 \\
\hline \multicolumn{11}{|c|}{${ }^{13} \mathrm{C}(3)$} \\
\hline & $B_{\mathrm{e}}$ calc. & $B_{0}$ calc. & $B_{\mathrm{e}}^{\text {calc. }}-B_{0}^{\text {calc. }}$ & $B_{0}{ }^{A} \_$exp. & $B_{e}{ }^{S E}$ & $B_{\mathrm{e}}^{\text {calc. }}$ & $B_{0}^{\text {calc. }}$ & $B_{\mathrm{e}}$ calc. $-B_{0}{ }^{\text {calc. }}$ & $B_{0}{ }^{A} \_$exp. & $B_{\mathrm{e}}^{\mathrm{SE}}$ \\
\hline $\boldsymbol{A}$ & 4819.549 & 4789.782 & 29.767 & 4836.050 & 4865.817 & 4756.752 & 4727.500 & 29.252 & 4788.202 & 4817.454 \\
\hline$B$ & 1630.475 & 1618.269 & 12.206 & 1605.265 & 1617.471 & 1625.692 & 1613.460 & 12.232 & 1626.604 & 1638.836 \\
\hline$C$ & 1227.582 & 1219.746 & 7.836 & 1214.757 & 1222.593 & 1220.773 & 1212.949 & 7.824 & 1223.834 & 1231.658 \\
\hline \multicolumn{11}{|c|}{${ }^{13} \mathrm{C}(4)$} \\
\hline & $B_{\mathrm{e}}^{\text {calc. }}$ & $B_{0}^{\text {calc. }}$ & $B_{e}{ }^{\text {calc. }}-B_{0}$ calc. & $B_{0} A_{-} \_$exp. & $B_{\mathrm{e}}^{\mathrm{SE}}$ & $B_{\mathrm{e}}^{\text {calc. }}$ & $B_{0}^{\text {calc. }}$ & $B_{\mathrm{e}}^{\text {calc. }}-B_{0}{ }^{\text {calc. }}$ & $B_{0}{ }^{A} \_$exp. & $B_{\mathrm{e}}^{\mathrm{SE}}$ \\
\hline $\boldsymbol{A}$ & 4804.683 & 4774.978 & 29.705 & 4836.050 & 4865.755 & 4794.643 & 4764.972 & 29.671 & 4826.662 & 4856.333 \\
\hline$B$ & 1604.443 & 1592.374 & 12.069 & 1605.265 & 1617.334 & 1608.896 & 1596.791 & 12.105 & 1609.508 & 1621.613 \\
\hline$C$ & 1211.824 & 1204.035 & 7.789 & 1214.757 & 1222.546 & 1213.720 & 1205.916 & 7.804 & 1216.589 & 1224.393 \\
\hline \multicolumn{11}{|c|}{${ }^{18} \mathrm{O}(7)$} \\
\hline & $B_{\mathrm{e}}^{\text {calc. }}$ & $B_{0}^{\text {calc. }}$ & $B_{e}^{\text {calc. }}-B_{0}^{\text {calc. }}$ & $B_{0} A_{-}$exp. & $B_{\mathrm{e}}^{\mathrm{SE}}$ & $B_{\mathrm{e}}^{\text {calc. }}$ & $B_{0}$ calc. & $B_{\mathrm{e}}^{\text {calc. }}-B_{0}{ }^{\text {calc. }}$ & $B_{0}{ }^{A} \_$exp. & $B_{\mathrm{e}}^{\mathrm{SE}}$ \\
\hline $\boldsymbol{A}$ & 4819.842 & 4790.142 & 29.700 & 4851.591 & 4881.291 & 4702.547 & 4673.242 & 29.305 & 4733.633 & 4762.938 \\
\hline$B$ & 1619.976 & 1607.885 & 12.091 & 1620.823 & 1632.914 & 1587.711 & 1576.071 & 11.640 & 1588.767 & 1600.407 \\
\hline C & 1221.640 & 1213.855 & 7.785 & 1224.637 & 1232.422 & 1195.756 & 1188.217 & 7.539 & 1198.819 & 1206.358 \\
\hline \multicolumn{11}{|c|}{${ }^{13} \mathrm{C}(8)$} \\
\hline & $B_{\mathrm{e}}{ }^{\text {calc. }}$ & $B_{0}^{\text {calc. }}$ & $B_{e}^{\text {calc. }}-B_{0}{ }^{\text {calc. }}$ & $B_{0} A_{-}$exp. & $B_{\mathrm{e}}^{\mathrm{SE}}$ & & & & & \\
\hline $\boldsymbol{A}$ & 4753.738 & 4724.761 & 28.977 & 4785.296 & 4814.273 & & & & & \\
\hline$B$ & 1607.783 & 1595.569 & 12.214 & 1608.374 & 1620.588 & & & & & \\
\hline$C$ & 1210.451 & 1202.633 & 7.818 & 1213.298 & 1221.116 & & & & & \\
\hline
\end{tabular}


Table S9b: "Unperturbed" experimental constants $\boldsymbol{B}_{0}{ }^{\mathrm{E}-\text { exp. }}$ deduced from the SFLAMS E species fits and the semi-experimental equilibrium rotational constants $\boldsymbol{B}_{\mathrm{e}}{ }^{\mathrm{SE}}=\left(\boldsymbol{B}_{0} \mathbf{A}_{-}\right.$exp. + $2 \boldsymbol{B}_{0}{ }_{-} \_$exp. $) / 3+\boldsymbol{B}_{\mathrm{e}}$ calc. $-\boldsymbol{B}_{0}$ calc. All values are given in $\mathrm{MHz}$.

\begin{tabular}{|c|c|c|c|}
\hline \multicolumn{2}{|c|}{ Isotopologue } & \multirow{2}{*}{$\begin{array}{c}\boldsymbol{B}_{0}{ }^{\mathrm{E}} \text { exp. } \\
4852.126\end{array}$} & \multirow{2}{*}{$\begin{array}{l}\boldsymbol{B}_{\mathrm{e}}^{\mathrm{SE}} \\
4881.98\end{array}$} \\
\hline${ }^{12} \mathrm{C}$ & $A$ & & \\
\hline & $B$ & 1631.238 & 1643.534 \\
\hline & $C$ & 1230.598 & 1238.501 \\
\hline \multirow[t]{3}{*}{${ }^{18} \mathrm{O}(1)$} & $A$ & 4753.716 & 4783.269 \\
\hline & $B$ & 1625.855 & 1638.012 \\
\hline & $C$ & 1221.141 & 1228.940 \\
\hline \multirow[t]{3}{*}{${ }^{13} \mathrm{C}(2)$} & $A$ & 4851.266 & 4875.961 \\
\hline & $B$ & 1631.332 & 1634.849 \\
\hline & $C$ & 1230.584 & 1233.145 \\
\hline \multirow[t]{3}{*}{${ }^{13} \mathrm{C}(3)$} & $A$ & 4788.237 & 4817.477 \\
\hline & $B$ & 1626.621 & 1638.847 \\
\hline & $C$ & 1223.837 & 1231.660 \\
\hline \multirow[t]{3}{*}{${ }^{13} \mathrm{C}(4)$} & $A$ & 4836.084 & 4865.778 \\
\hline & $B$ & 1605.282 & 1617.345 \\
\hline & $C$ & 1214.760 & 1222.548 \\
\hline \multirow[t]{3}{*}{${ }^{13} \mathrm{C}(5)$} & $A$ & 4826.694 & 4856.354 \\
\hline & $B$ & 1609.526 & 1621.625 \\
\hline & $C$ & 1216.592 & 1224.395 \\
\hline \multirow[t]{3}{*}{${ }^{13} \mathrm{C}(6)$} & $A$ & 4851.625 & 4881.314 \\
\hline & $B$ & 1620.841 & 1632.926 \\
\hline & $C$ & 1224.640 & 1232.424 \\
\hline \multirow[t]{3}{*}{${ }^{18} \mathrm{O}(7)$} & $A$ & 4733.665 & 4762.959 \\
\hline & $B$ & 1588.785 & 1600.419 \\
\hline & $C$ & 1198.822 & 1206.360 \\
\hline \multirow[t]{3}{*}{${ }^{13} \mathrm{C}(8)$} & $A$ & 4785.327 & 4814.294 \\
\hline & $B$ & 1608.391 & 1620.600 \\
\hline & $C$ & 1213.301 & 1221.118 \\
\hline
\end{tabular}


Table S10: Rovibrational corrections at the B2PLYP-D3/6-311+G(3df,2pd) level of theory, semi-experimental equilibrium rotational constants, and residuals of the fit to determine the $r_{0 \rightarrow \mathrm{e}}^{\mathrm{SE}}$ structure. All values are given in $\mathrm{MHz}$.

\begin{tabular}{|c|c|c|c|c|}
\hline \multicolumn{2}{|c|}{ Isotopologue } & \multirow{2}{*}{$\begin{array}{l}\Delta B \\
31.102\end{array}$} & \multirow{2}{*}{$\begin{array}{c}\text { B } \\
4883.223\end{array}$} & \multirow{2}{*}{$\begin{array}{r}\text { Obs. - Calc. } \\
-0.001\end{array}$} \\
\hline${ }^{12} \mathrm{C}$ & $A$ & & & \\
\hline & $B$ & 12.484 & 1643.720 & -0.016 \\
\hline & $C$ & 8.565 & 1239.154 & -0.009 \\
\hline \multirow[t]{3}{*}{${ }^{18} \mathrm{O}(1)$} & $\boldsymbol{A}$ & 30.805 & 4784.520 & 0.000 \\
\hline & $B$ & 12.387 & 1638.240 & 0.001 \\
\hline & $C$ & 8.484 & 1229.616 & 0.001 \\
\hline \multirow[t]{3}{*}{${ }^{13} \mathrm{C}(2)$} & $\boldsymbol{A}$ & 30.961 & 4882.229 & 0.000 \\
\hline & $B$ & 12.377 & 1643.699 & -0.025 \\
\hline & $C$ & 8.491 & 1239.076 & -0.016 \\
\hline \multirow[t]{3}{*}{${ }^{13} \mathrm{C}(3)$} & $\boldsymbol{A}$ & 30.510 & 4818.742 & 0.000 \\
\hline & $B$ & 12.394 & 1639.014 & -0.004 \\
\hline & $C$ & 8.474 & 1232.302 & -0.002 \\
\hline \multirow[t]{3}{*}{${ }^{13} \mathrm{C}(4)$} & $A$ & 30.970 & 4867.050 & 0.000 \\
\hline & $B$ & 12.231 & 1617.511 & 0.001 \\
\hline & $C$ & 8.430 & 1223.181 & 0.001 \\
\hline \multirow[t]{3}{*}{${ }^{13} \mathrm{C}(5)$} & $\boldsymbol{A}$ & 30.865 & 4857.557 & 0.000 \\
\hline & $B$ & 12.319 & 1621.843 & 0.001 \\
\hline & $C$ & 8.471 & 1225.054 & 0.001 \\
\hline \multirow[t]{3}{*}{${ }^{13} \mathrm{C}(6)$} & $\boldsymbol{A}$ & 30.863 & 4882.486 & 0.000 \\
\hline & $B$ & 12.318 & 1633.157 & 0.078 \\
\hline & $C$ & 8.464 & 1233.095 & 0.046 \\
\hline \multirow[t]{3}{*}{${ }^{18} \mathrm{O}(7)$} & $\boldsymbol{A}$ & 30.152 & 4763.815 & 0.000 \\
\hline & $B$ & 12.101 & 1600.883 & 0.002 \\
\hline & $C$ & 8.312 & 1207.125 & 0.001 \\
\hline \multirow[t]{3}{*}{${ }^{13} \mathrm{C}(8)$} & $\boldsymbol{A}$ & 30.150 & 4815.478 & 0.000 \\
\hline & $B$ & 12.400 & 1620.791 & 0.001 \\
\hline & $C$ & 8.470 & 1221.762 & 0.001 \\
\hline
\end{tabular}


Table S11: Rotational constants of 2-acetyl-5-methylfuran, furfural, and 5-methylfurfural calculated at the B3LYP-D3BJ/6-311G(d,p), B3LYP-D3BJ/6-311+G(d,p), and B3LYP$\mathrm{D} 3 \mathrm{BJ} / 6-311++\mathrm{G}(\mathrm{d}, \mathrm{p})$ levels of theory.

\begin{tabular}{|c|c|c|c|c|c|c|}
\hline Method/Basis Set & $\begin{array}{c}\text { A / } \\
\mathrm{MHz}\end{array}$ & $\begin{array}{l}\Delta \boldsymbol{A} / \\
\mathrm{MHz}\end{array}$ & $\begin{array}{c}B / \\
\mathrm{MHz}\end{array}$ & $\begin{array}{l}\Delta B / \\
\mathrm{MHz}\end{array}$ & $\begin{array}{c}C / \\
\mathrm{MHz}\end{array}$ & $\begin{array}{l}\Delta C / \\
\mathrm{MHz}\end{array}$ \\
\hline & \multicolumn{6}{|c|}{ anti-furfural } \\
\hline B3LYP-D3BJ/6-311G(d,p) & 8228.2 & 36.4 & 2045.7 & -0.2 & 1638.4 & 1.2 \\
\hline B3LYP-D3BJ/6-311+G(d,p) & 8233.9 & 42.1 & 2040.4 & -5.5 & 1635.2 & -2.0 \\
\hline B3LYP-D3BJ/6-311++G(d,p) & 8234.1 & 42.3 & 2040.3 & -5.6 & 1635.2 & -2.0 \\
\hline \multirow[t]{2}{*}{ Expt. $X_{0}$} & 8191.8 & & 2045.9 & & 1637.2 & \\
\hline & \multicolumn{6}{|c|}{ syn-furfural } \\
\hline B3LYP-D3BJ/6-311G(d,p) & 8175.7 & 32.0 & 2095.4 & -3.3 & 1667.9 & -1.0 \\
\hline B3LYP-D3BJ/6-311+G(d,p) & 8180.7 & 37.0 & 2091.2 & -7.5 & 1665.5 & -3.4 \\
\hline B3LYP-D3BJ/6-311++G(d,p) & 8181.0 & 37.3 & 2091.0 & -7.7 & 1665.3 & -3.6 \\
\hline \multirow[t]{2}{*}{ Expt. $X_{0}$} & 8143.7 & & 2098.7 & & 1668.9 & \\
\hline & \multicolumn{6}{|c|}{ anti-5-methylfurfural } \\
\hline B3LYP-D3BJ/6-311G(d,p) & 6352.6 & 46.2 & 1312.1 & -2.7 & 1094.9 & -0.7 \\
\hline B3LYP-D3BJ/6-311+G(d,p) & 6332.4 & 26.0 & 1311.1 & -3.7 & 1093.6 & -2.0 \\
\hline B3LYP-D3BJ/6-311++G(d,p) & 6332.2 & 25.9 & 1311.1 & -3.8 & 1093.6 & -2.0 \\
\hline \multirow[t]{2}{*}{ Expt. $X_{0}$} & 6306.4 & & 1314.8 & & 1095.6 & \\
\hline & \multicolumn{6}{|c|}{ syn-5-methylfurfural } \\
\hline B3LYP-D3BJ/6-311G(d,p) & 4773.2 & 18.7 & 1494.0 & -4.2 & 1145.9 & -1.5 \\
\hline B3LYP-D3BJ/6-311+G(d,p) & 4779.3 & 24.8 & 1490.5 & -7.7 & 1144.3 & -3.2 \\
\hline B3LYP-D3BJ/6-311++G(d,p) & 4780.0 & 25.5 & 1490.3 & -7.9 & 1144.2 & -3.3 \\
\hline \multirow[t]{2}{*}{ Expt. $X_{0}$} & 4754.5 & & 1498.2 & & 1147.5 & \\
\hline & \multicolumn{6}{|c|}{ anti-2-acetyl-5-methylfuran } \\
\hline B3LYP-D3BJ/6-311G(d,p) & 3707.5 & 7.9 & 1124.9 & -1.0 & 872.4 & -0.5 \\
\hline B3LYP-D3BJ/6-311+G(d,p) & 3704.8 & 5.1 & 1124.4 & -1.6 & 871.9 & -1.0 \\
\hline B3LYP-D3BJ/6-311++G(d,p) & 3704.9 & 5.2 & 1124.4 & -1.5 & 871.9 & -1.0 \\
\hline \multirow[t]{2}{*}{ Expt. $X_{0}$} & 3699.7 & & 1126.0 & & 872.9 & \\
\hline & \multicolumn{6}{|c|}{ syn-2-acetyl-5-methylfuran } \\
\hline B3LYP-D3BJ/6-311G(d,p) & 3918.9 & 5.0 & 1094.4 & -1.1 & 864.7 & -0.8 \\
\hline B3LYP-D3BJ/6-311+G(d,p) & 3922.1 & 8.3 & 1092.9 & -2.7 & 863.9 & -1.6 \\
\hline B3LYP-D3BJ/6-311++G(d,p) & 3922.2 & 8.3 & 1092.9 & -2.7 & 863.9 & -1.6 \\
\hline Expt. $X_{0}$ & 3913.9 & & 1095.6 & & 865.5 & \\
\hline
\end{tabular}

